\title{
FIRST NOTES ON THE PHONOLOGY AND CLASSIFICATION \\ OF THE BANGRU LANGUAGE OF INDIA ${ }^{1}$ Timotheus A. Bodt \& Ismael Lieberherr \\ University of Bern, Switzerland/Tezpur University, India \\ Corresponding author: Tim Bodt, timintibet@hotmail.com
}

\begin{abstract}
This is the first description of the Bangru language spoken in a remote corner of Kurung Kumey district, Arunachal Pradesh, Northeast India. On the basis of our data and analysis, we identify Bangru as a Tibeto-Burman language with as its closest genetic relatives Miji and Hruso, spoken further to the southwest in East and West Kameng districts. Based on these preliminary data and their analysis, Bangru is described as having 18 distinctive consonants, seven distinctive simple vowels and three distinctive diphthong vowels. The basic syllable structure is $\mathrm{C}_{\mathrm{i}}(\mathrm{G}) \mathrm{V}\left(\mathrm{C}_{\mathrm{f}}\right)$. Bangru is furthermore characterised by the retention of Proto-Tibeto-Burman prefixes as reduced syllables. This paper also provides additional evidence for the validity of Shafer's 'Hruso', with Hruso itself as its most aberrant member.
\end{abstract}

Keywords: Bangru, Tibeto-Burman, Trans-Himalayan, Miji, Aka, Hruso, Hrusish, genetic classification, phonology.

\section{INTRODUCTION}

In this paper we survey the existing literature about the Bangru language, propose a provisional phonology of the languages, and discuss its possible genetic affiliation.

\subsection{ETHNOLINGUISTIC OVERVIEW}

The vast majority of the population of Kurung Kumey district, around $90 \%$, speak Nyasang ${ }^{2}$. The Bangru are administratively part of the larger Nyishi Scheduled

\footnotetext{
1 This paper is the result of fieldwork conducted in Sarli circle, Kurung Kumey district, Arunachal Pradesh in December 2013. The authors wish to acknowledge the assistance of local host and consultant Mr. Tafiap Yangfo and his family, guide and consultant Mr. Tame Ramya, the cooperation of Tezpur University students Eshani Baishya, Sansuma Brahma, Nawaf Zahdaan Helmi, Diksha Konwar, Rahul Matela and Pinaz Mirza and Tezpur University project staff Ms. Nupur Sinha and the kind coordination by Mrs. Madhumita Barbora of the Department of English and Foreign Languages. This research would not have been possible without the patient contributions of local informants Pisa Meji, Pisa Nikma, Bengia Yapang, Pisa Niglar, Milli Kafa, Pisa Tamang and Pisa Chachung. The paper also benefited from the value comments and suggestions by two anonymous referees as well as Prof. Dr. Graham Thurgood, California State University and Mr. Yeshy T. Sotrug, Bern University.

${ }^{2}$ Nyasang is the local variety of the language more commonly known as Nyishi, Nishi, Nishing or Bengni, belonging to the Western Tani group.
} 
Tribe and as a result of intense contact with the majority Nyasang population they share many cultural characteristics. Within remote Sarli circle, the Bangru constitute $40 \%$ of the population (Tame, 2011). There is also a small Puroik community in the circle. The Bangru number around 1,500 people.

The Bangru inhabit the traditional lawje: ne:pe: rạle: katã̃n 'thirteen Bangru villages'. They consider this area as their original homeland and claim descent from two sons of 'ase: ladzuwje: 'Grandmother Sun'. The Bangru call themselves ta:da or ta: $\overline{d z} u:$ banru: and belong to five clans, the original padzo: $\overline{d z} u$ : Pisa, malo: $\overline{d z} u:$ Melo, tagan $\overline{d z} u$ : Tagang and malidzu: Mili clans descending from one of the sons, and the sa:pa Sape clan descending from a bride who came later from Tibet. The Bangru believe that the other brother migrated to the Lada area of East Kameng district where he became the ancestor of the wa:du: banru: or the Miji people. Like most people of the state, the Bangru practise shifting cultivation and rely heavily on the forest resources. The original Bangru belief system, now largely replaced by Christianity, accorded paramount importance to the sun and moon, who are respectfully addressed as 'alo: labã̃ 'our grandfather moon' and 'ase: lad̄au:wje: 'our grandmother son', even in daily speech.

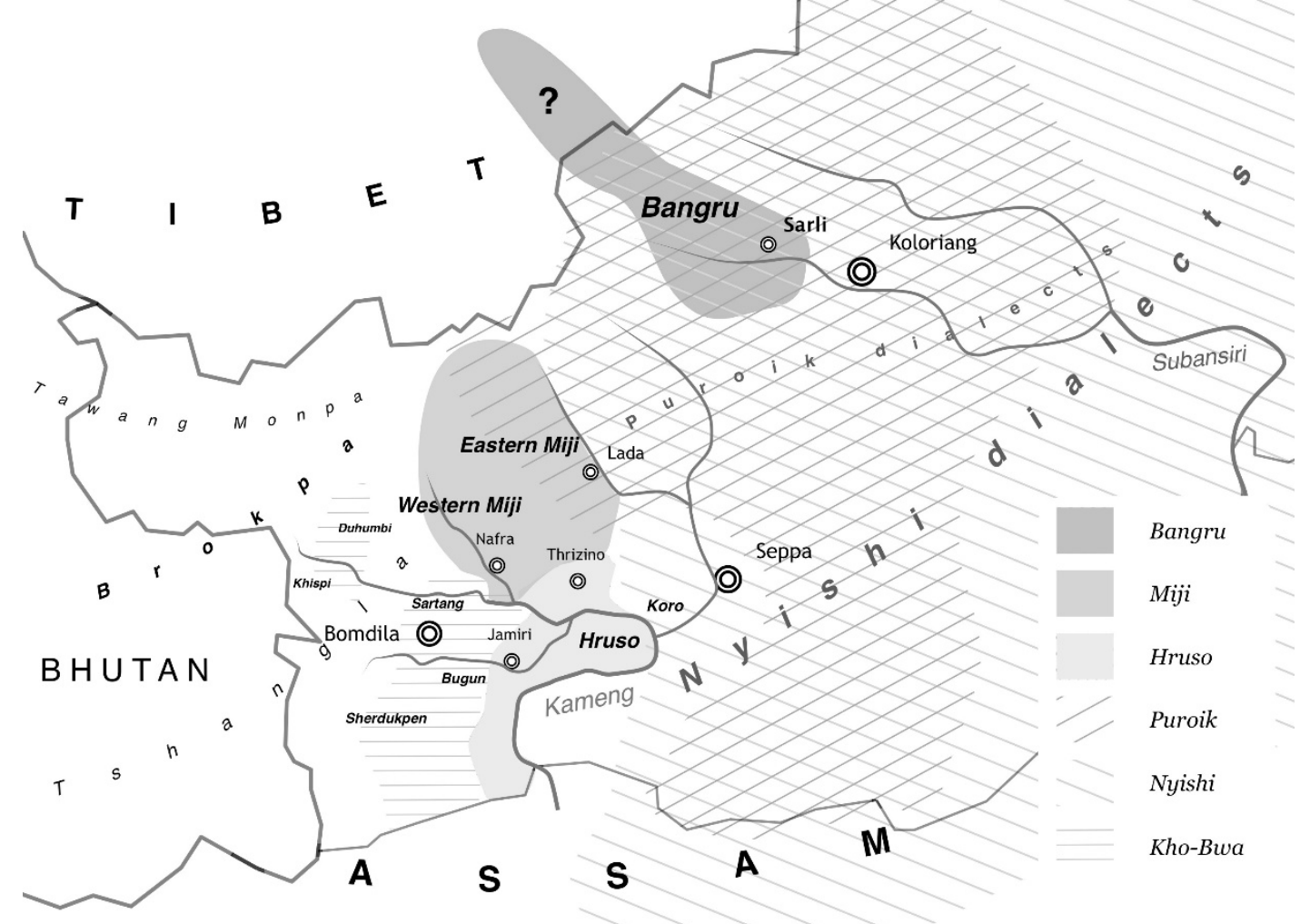

Figure 1. Bangru and its neighbours. 


\subsection{PREVIOUS LITERATURE}

Previous literature about the Bangru and their language is scant. Sun (1993: 348) was the first to report a Bangru population of 1,000 people. Sun wrote about Bangru that

"There is at least one more Hrusish language in Arunachal Pradesh, namely the language of the Bangru tribe of North-western Upper Subansiri district. Publications on the Bangru language are completely non-existent. Our limited fieldwork data on Bangru reveals such striking resemblances between Bangru and Dhammai that they may even turn out to be dialects of the same language".

And in the footnote on the same page

"The Bangru (autonym Levai $/ l \partial^{31} v e^{55} /$ ) tribe consists of about a thousand souls whose villages are distributed in the Lagong area along the Tibetan-Indian border (Anonymous 1989:248). Note the similarity between the name Levai and the Miji autonym Dhammai (/dum-mai/). It is possible that the Levai represents a northeastern subbranch of the Mijis of Eastern Kameng. The name Bangru (burn-ru/) is a Bengni exonym; cf. also the Sulung exonym of Levai: Buzwa $\left.\left(\mathrm{bu}^{33} \mathrm{zwa}^{53}\right)\right)^{\prime \prime}$.

Sun was thus the first to establish a possible relation between Bangru and the Hrusish languages. The Hrusish languages, including the Miji varieties of East and West Kameng and Hruso (Aka), were identified as a subgroup by Shafer (1947, 1955) based on Hruso and West Kameng Miji data. Hruso descriptions can be found in Anderson (1896), Schubert (1964), Simon (1993 [1970]) and Abraham et al. (2005). Miji descriptions can be found in Shafer (1947), Simon (1979) and Abraham et al. (2005).

There appears to be no print version of the one thousand Bangru words that Sun collected from his Puroik consultant in Tibet (footnote 256 in Sun 1993: 348). In 2003, Li published a short description of the phonology, morphology, basic grammar and vocabulary of $l a P^{31}$ wai ${ }^{55}$ Bangru, based on two Bangru informants. Li indicates that there are six ethnic Bangru in doǔ yuè yī village on the Tibetan side of the border, out of which only three speak the Bangru language, all aged 50 years or older: this speech community is obviously moribund, if not extinct by now. He mentions that it is estimated that another 2,000 Bangru speakers inhabit various villages south of the Line of Actual $\mathrm{Control}^{3}$. A comparison between our data and the data in Li (2003) shows $50 \%$ of lexemes with same root and same form, $30 \%$ of lexemes with same root but different form, and $20 \%$ of lexemes with different root.

\footnotetext{
${ }^{3}$ The authors wish to thank Mr. Yeshy T. Sotrug, University of Bern for his translation of this description.
} 
In 2011, Tame's M. Phil. dissertation was the first study on the Bangru of Arunachal Pradesh, which included a word list, followed by several articles (2012 and 2013). Tame's word list is reproduced without proper attribution in Blench (2014).

Although recently doubts were raised on the validity of this subgroup and on the affiliation of Hruso with the Tibeto-Burman ${ }^{4}$ language family as a whole (Blench and Post 2014), Anderson (2014) recently presented an initial overview that the Hrusish languages should in fact be considered to belong to this family. Although he proposes the name 'South-eastern Kamengic', we prefer to continue to use the name Hrusish. For the purpose of this article, we follow Shafer's initial Hrusish hypothesis, with the addition of Bangru. This preliminary family tree is schematically represented in figure 2 .

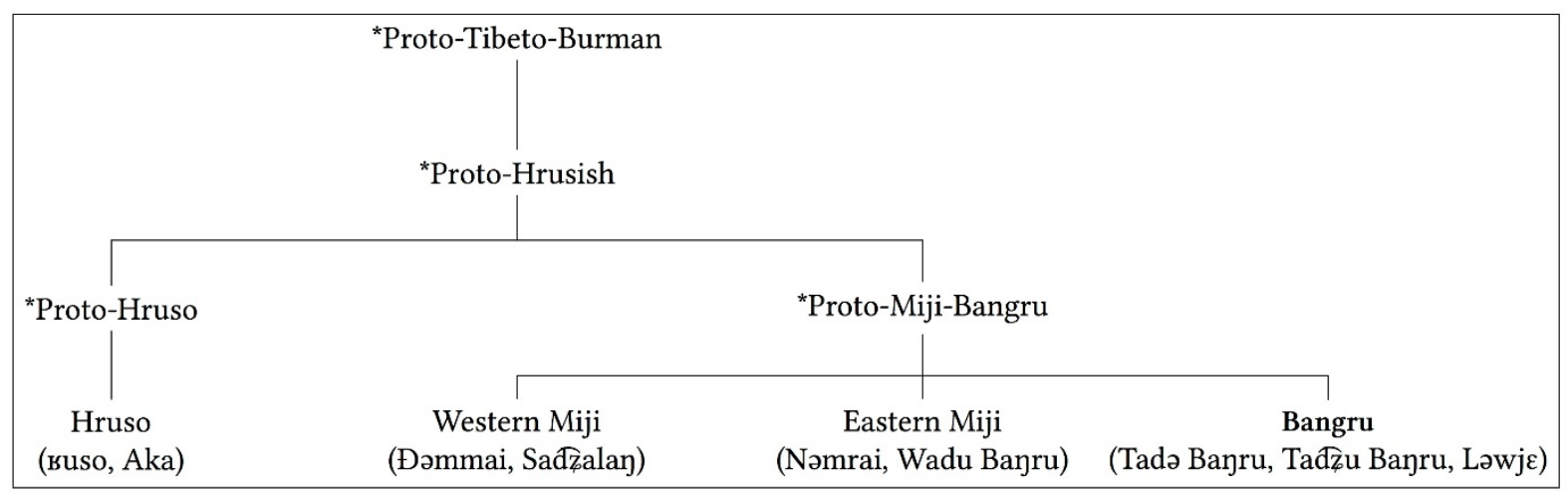

Figure 2. Preliminary Hrusish family tree.

\section{DATA}

This description is based on original field research conducted in sa:ta: Sate village and Sarli circle headquarter in December 2013. Our informants were six mother tongue speakers and one Nyasang speaker who learnt Bangru as a second language. Three of our informants were female, and four were male. One speaker was over 70 years of age, three speakers were over 50 years of age, two speakers were between 30 and 50 years of age, and one speaker was a teenager. Six speakers belonged to the Pisa clan and one speaker belonged to the Mili clan. Although we used Hindi as direct contact language, we also relied on two Nyasang speakers native to Kurung Kumey district with some knowledge of Bangru and a good knowledge of both Hindi and English.

We elicited and recorded lexical items from a list with 441 entries occurring on the Swadesh 100 and 200 word lists, Matisoff's CALMSEA list (Matisoff 1978: 284), the Leipzig-Jakarta list (Haspelmath and Tadmore 2009) and the SIL list from Abraham et al.'s 2005 linguistic variety survey of western Arunachal

\footnotetext{
${ }^{4}$ In von Klaproth's 1823 original sense, i.e. Tibetan, Burmese, Chinese and all languages that can be demonstrated to be genetically related to these. Recently, van Driem $(2011,2014)$ suggested the neutral, geographical term Trans-Himalayan in recognition of the wide linguistic variety found among populations straddling the Himalayan divide.
} 
Pradesh. We also recorded and transcribed any additional lexical items that speakers mentioned. Furthermore, we elicited a limited number of basic phrases and sentences. Finally, we recorded two origin stories.

Appendix C contains a list with secondary sources we used for comparative data of other languages including the abbreviations used in the remainder of this paper. On basis of Bangru and Miji speaker information about mutual intelligibility, we presume that Bangru and Eastern Miji might be more closely related than Bangru and Western Miji. Abraham et al.'s lexical similarity scores (2005: 13) indicate that 'Miji' itself exhibits considerable internal variation. Although this will not be the focus of this paper, for our comparative purposes it led us to group the data from Simon and data of speaker C, D and e from Abraham et al. (2005) together. These four speakers appear to represent the western variety of Miji spoken in Nafra and Thrizino circles of West Kameng district by the people known by the endonyms of sadzalan Sajalang or đəmmai Dhəmmai, henceforth referred to as Western Miji. Speakers A and B represent the eastern variety of Miji spoken in Lada circle of East Kameng by the people known as namrai Nəmrai, henceforth referred to as Eastern Miji. The suso Hruso data in Simon (1993 [1970]) and Abraham et al. (2005) were both from the most accessible Hruso village, $k^{h}$ utso Jamiri. As a result of two independent sources, Abraham et al. (2005) and Simon (1979), we were able to find 421 of our Bangru entries in the Western Miji varieties, 280 entries of Bangru entries in the Eastern Miji varieties, and 273 of the Bangru entries in the Hruso sources.

\section{PRELIMINARY PHONOLOGY}

The following sections provide a concise overview of the segmental phonology of Bangru. Because of the preliminary nature of our research, this overview should be considered as a first initial sketch. We provide minimal pairs wherever possible.

\subsection{CONSONANT PHONEMES}

Our data show that Bangru has a relatively simple inventory of 18 consonant phonemes in eight places and eight manners of articulation, summarised in Figure 3.

\begin{tabular}{|c|c|c|c|c|c|c|c|}
\hline $\begin{array}{l}\text { manner of } \\
\text { articulation }\end{array}$ & bilabial & $\begin{array}{l}\text { labio- } \\
\text { velar }\end{array}$ & $\begin{array}{l}\text { alveo- } \\
\text { lar }\end{array}$ & post-alveolar & $\begin{array}{c}\text { pala- } \\
\text { tal }\end{array}$ & velar & glottal \\
\hline nasal & $\mathrm{m}$ & & $n$ & & $n$ & $\eta$ & \\
\hline stop & $p \quad b$ & & $\begin{array}{ll}\mathrm{t} & \mathrm{d}\end{array}$ & & & $\mathrm{k}$ & \\
\hline affricate & & & & $\overline{d z}$ & & & \\
\hline fricative & & & $\mathrm{s}$ & & & & $\mathrm{h}$ \\
\hline approximant & & $w$ & & & j & & \\
\hline
\end{tabular}




\begin{tabular}{ll}
\hline lat. approx. & $\mathrm{I}$ \\
\cline { 1 - 1 } & $\mathrm{r}$ \\
\hline trill
\end{tabular}

Figure 3. Bangru consonant phonemes

Bangru has three unvoiced plosive consonant phonemes in three places of articulation, velar, alveolo-dental and bilabial, $/ \mathrm{k}, \mathrm{t}, \mathrm{p} / \mathrm{and}$ their three voiced counterparts $/ \mathrm{g}, \mathrm{d}, \mathrm{b} /$.
/k/ vs. /g/ ku: 'cook meat' vs. magu: 'chin'; go: 'wood' vs. ko: 'stab, punch'; maki: 'dry' vs. gi: 'stand'
/t/ vs. /d/ to: 'bite' vs. do: 'chicken'; du: 'have, exist' vs. matu: 'tooth'
/p/ vs. /b/ pi: 'awake' vs. bi: 'run'; pa: 'kick' vs. ba: 'bamboo'

Bangru has two distinctive fricative phonemes, unvoiced alveolar fricative /s/ and the unvoiced glottal fricative $/ \mathrm{h} /$. Both fricatives occur only in syllable-initial position. Before the close, back rounded vowel $/ \mathrm{u} /$ and the diphthong /ua/ the unvoiced alveolar fricative has as allophone in free variation the unvoiced postalveolar fricative [6].

/s/ vs. /h/ mase: 'other's grandmother' vs. mahe: 'empty'; san 'live, grow up’ vs. han 'this'

We found two distinctive affricate phonemes in Bangru, the unvoiced alveolopalatal affricate $/ \overline{\mathrm{t} \epsilon} /$ and the voiced alveolo-palatal affricate $/ \overline{\mathrm{d} z} /$. The voiced alveolar affricate $/ \bar{d} z_{0} /$ can precede all distinctive monophthong and diphthong vowel phonemes and occurs in free alternation with the voiced postalveolar affricate $[\bar{d} 3]$ and has two speaker-dependant irregular allophones, voiced alveolar fricative $[\mathrm{z}]$ and voiced alveolar affricate $[\overline{\mathrm{dz}}]$.

The unvoiced alveolo-palatal affricate $/ \bar{t} \zeta /$ occurs in a limited set of lexemes and its realisation is remarkably divergent across lexemes and speakers. In onset position, the phoneme can be realised as unvoiced alveolo-palatal affricate [tढ] as unvoiced postalveolar affricate $\widehat{[\mathrm{t} f]}$ or as unvoiced alveolar affricate $\widetilde{\mathrm{ts}}]$. In coda position, we found realisation as both unvoiced and unvoiced aspirated postalveolar and alveolo-palatal affricates $\left[\overline{\mathrm{t} \int} \sim \overline{\mathrm{t} \int^{\mathrm{h}}} \sim \overline{\mathrm{t} \epsilon} \sim{\overline{\mathrm{t} \epsilon^{h}}}^{\mathrm{h}}\right.$. Whereas in coda position some speakers realise one of the affricate allophones, other speakers realise the last syllable of the word with an epenthetic syllable-final breathy voice vowel [i]. Examples are mainly the demonstratives and the noun [məpoţ $~$

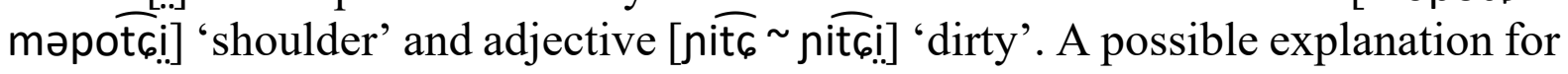
this may be language contact with Nyasang. The iambic rhythm of Bangru places stress on the second syllable and reduces the initial syllable, but the trochaic rhythm of Nyasang stresses the initial syllable and reduces the final syllable.

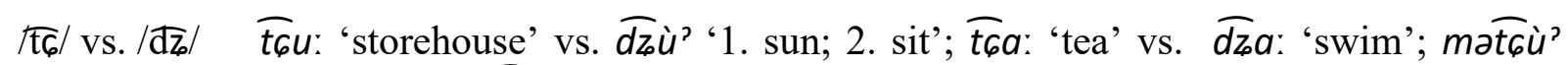
'wing' vs. mad̄au: 'child' 
Bangru has nasals in four places of articulation, bilabial $/ \mathrm{m} /$, alveolar $/ \mathrm{n} /$, palatal $/ \mathrm{n} /$ and velar $/ \mathrm{y} /$. The nasal $/ \mathrm{y} /$ occurs mainly as coda, with a few noted exceptions such as panu: 'five', nua 'melt' and sanai sani: 'ant'. The other nasals do not occur as coda in native lexemes, except for the bilabial nasal $/ \mathrm{m} /$ in probable loans such as 'amam 'fat, stout' $(<$ Puroik amam) and saram 'otter' $(<$ Nyasang saram). The alveolar nasal is sometimes palatalised before front vowels. Historical presence of syllable final nasals is a strong trigger for nasalisation of the preceding monophthong or diphthong vowel phoneme.

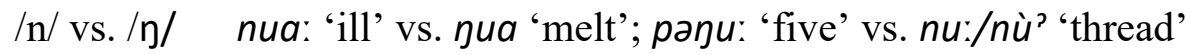

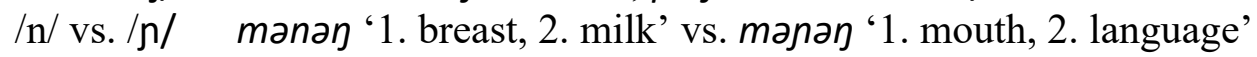

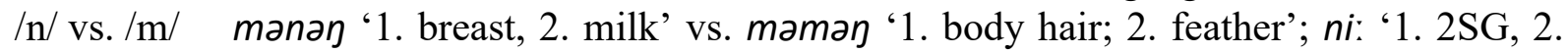
know' vs. mi: 'think'

Bangru has three approximants in three places of articulation, the palatal approximant $/ \mathrm{j} /$, the lateral approximant $/ 1 /$ and the voiced labiovelar continuant (labialised velar approximant) $/ \mathrm{w} /$. All three occur in onset position and the palatal approximant also occurs in initial consonant clusters. The voiced labiovelar continuant has an allophone in free alternation, the voiced labiodental fricative $[\mathrm{v}]$. Transcriptions maintain the exact realisation. Bangru has a distinctive alveolar trill $/ \mathrm{r} /$.

/j/vs. /w/ ja: 'blood' vs. wa: 'shifting cultivation plot'

/j/ vs. /1/ ja: 'blood' vs. la: 'sell'; ja:/ja' 'spittle' vs. la: 'take'

/r/ vs. /1/ ru: '1. salt, 2. do/make' vs. lu: 'boil water', maro: 'liver' vs. malo: 'hard'

\subsection{VOWEL PHONEMES}

The distinctive monophthong vowels of Bangru are a close, front unrounded vowel /i/ [i], a close, back rounded vowel /u/ [u], a close-mid, near-front unrounded vowel/e/ [e], the schwa/ə/ [ə] with close, central unrounded allophone [i], the open-mid, near-front unrounded vowel $/ \varepsilon /[\varepsilon]$ with allophones near-front

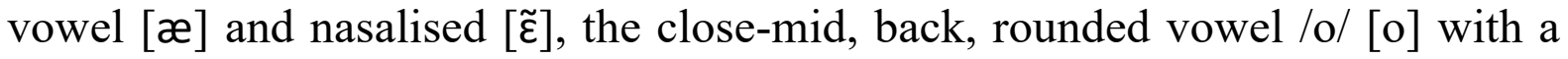
nasalised allophone [õ] and an open-mid, back rounded allophone [0] and the open, central unrounded vowel /a/ [a]. The origin of the Bangru nasalised vowels is the result of a process of elision of the syllable-final nasal consonants $/ \mathrm{n}, \mathrm{n}, \mathrm{m} /$, as described in section 4.5. This is supported by the fact that some speakers still realise a nasal consonant following a nasalised vowel, an observation transcribed as $\left[\left(\mathrm{C}_{\mathrm{i}}\right) \tilde{\mathrm{v}}(\mathrm{y} / \mathrm{n})\right]$. Vowel length can be predicted by phonotactic position in most cases, with vowels in open syllables longer than vowels in closed syllables. Noted exceptions are the open, central, unrounded vowel /a/ and the schwa/a/, which are always short in prefixes even if these syllables are open. Vowel length, pitch 
and glottal stop interact in intricate ways, as described in section 3.3. For clarity, long vowels are marked with [:] and /:/ in phonetic and phonemic transcriptions respectively.

In onset position, the Bangru vowels are inherently pre-glottalised. Of the three vowels that occur as onsets, /a/, /i/ and / $\tilde{\varepsilon} /$, the vowel /a/ can be found in syllableinitial position quite commonly, mainly as the prefix a- for own relatives and adjectives, but syllable-initially and monosyllabically the vowels $/ \mathrm{i} /$ and the $/ \tilde{\varepsilon} /$ have only been attested in the lexemes $\tilde{\varepsilon}:$ 'paddy rice', i: 'deep', i:rin 'window'.

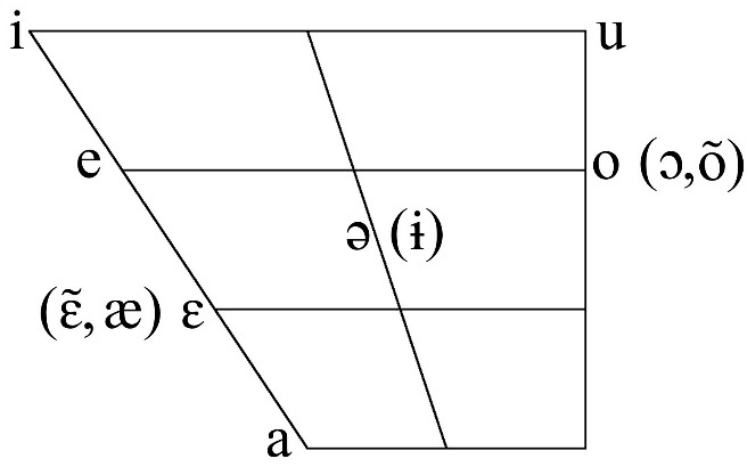

Figure 4. Bangru monophthong vowel phonemes.

/a/ vs. /u/ ta: '1. dig, 2. wound' vs. matu: 'tooth'; là̀' 'fireplace' vs. lù' 'month'; ja: 'blood' vs. ju: 'turquoise'

/a/ vs. /ə/ sa: 'pull' vs. sa: '1. cow, 2. iron'; $\overline{d z} a$ : 'swim' vs. $\overline{d z} ə$ a: 'frog'

/a/ vs//e/ ta: '1. dig, 2. wound' vs. te: 'speak'

/a/ vs. /e/ ama: 'sister (elder)' vs. $m \varepsilon:$ 'fire'; $p a:$ 'kick' vs. $p \varepsilon:$ 'swallow' re:

/a/ vs. /o/ pa: 'kick' vs. po: 'love'; da: 'make' vs. do: 'chicken'

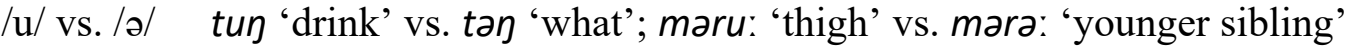

/u/vs. /o/ putढ़! '(that) down there' vs. potढ़! 'shoulder'; wu: 'put' vs. lawo: 'sago palm'

/i/vs. /a/ mari: 'bone' vs. mara: 'younger sibling'

/i/ vs. /e/ maki: 'dry' vs. ke: 'cry'; pi: 'awake' vs. pe: 'vegetable'

/i/ vs. / / / nawi: 'man' vs. nawe: 'woman'; pi: 'awake' vs. pe: 'swallow'

/a/vs. /e/ dza: 'frog' vs. dze: '1. sleep, 2.3PL'

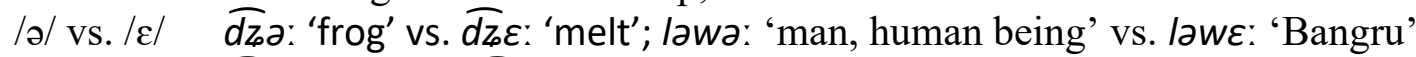

/a/ vs. /o/ dza: 'frog' vs. dzo: 'burn'; ase: 'grandmother' vs. aso: 'different, other'

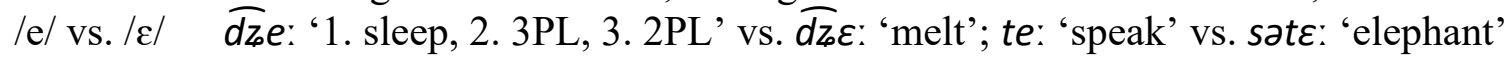

Bangru has three diphthongs: rising diphthongs [ai] and [ci] and falling diphthong [ua]. The rising diphthong [ai] is a short diphthong starting with an open, central unrounded vowel /a/, and ending in a close, front unrounded vowel /i/. In some speakers, this diphthong is monophthongised to open-mid, near-front unrounded vowel $/ \varepsilon /$. The rising diphthong [عi] is a short diphthong starting with an openmid, near-front unrounded vowel $/ \varepsilon /$ and ending in a close, front unrounded vowel /i/. In absence of convincing minimal pairs, we consider the nasalised variety of this diphthong [ $\widetilde{\varepsilon}]$ as an allophonic variation, probably conditioned by the presence of a syllable-final nasal. The rising diphthongs have been attested in 
open syllables and intervocalically with diphthong [ai] being more common than diphthong [ri].

The falling diphthong [ua] is a long diphthong starting with a close, back rounded vowel $/ \mathrm{u} /$ and ending in an open, central unrounded vowel $/ \mathrm{a} /$. This diphthong has only been attested in open syllables. Alternative realisations include a falling diphthong [oa] and, particularly in careful speech, an initial consonant cluster Cwa.

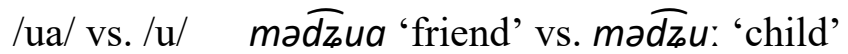

/ua/ vs. /a/ gua 'rot' vs. ga: 'clothing'; lua loa 'wind' vs. la: 'sell'

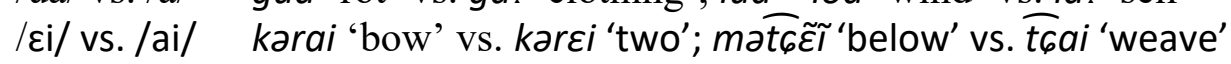

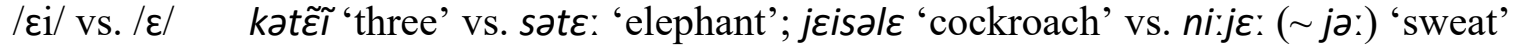

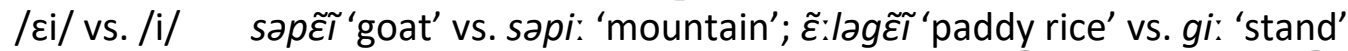

/ai/vs. /i/ bai 'give' vs. bi: 'run'; tai 'die' vs. ti: 'who'; tढai 'excrete' vs. $\overline{t \epsilon i}$ i: 'liquor'

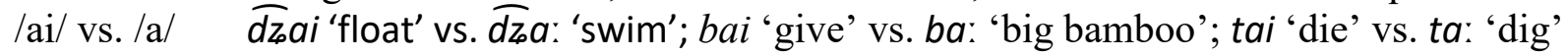

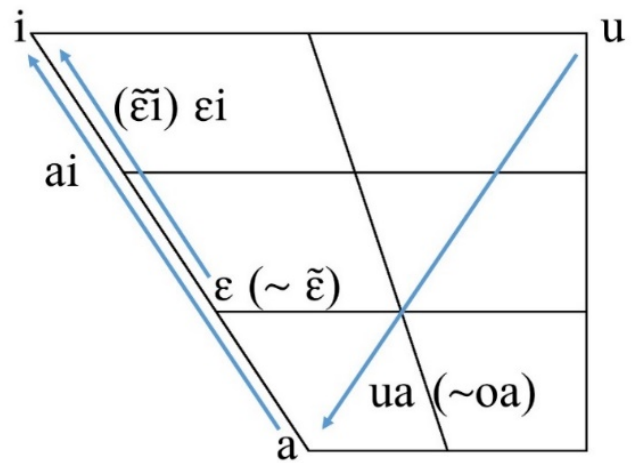

Figure 5. Bangru diphthong vowel phonemes

\subsection{CODAS, PITCH AND VOWEL LENGTH}

Bangru has a number of minimal pairs contrasting a long vowel with level pitch and slight final aspiration or breathy voice $\left[\overline{\mathrm{v}}: \sim \overline{\mathrm{v}}^{\mathrm{h}}\right.$ : $]$ versus a short vowel with

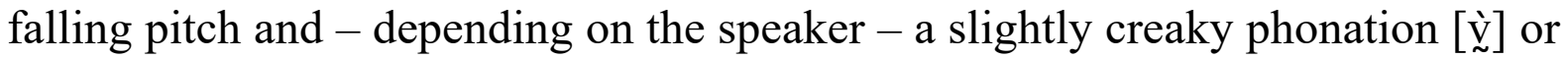
a syllable final glottal closure or glottal stop [ $\left.\grave{\mathrm{v}}^{2} \sim \grave{\mathrm{v}} \mathrm{?}\right]$. We observed this for both monophthong vowels and diphthong vowels. A number of minimal and nearminimal pairs are presented in table 1. Reconstructed Proto-Tibeto-Burman and Miji cognates have been provided if illustrative for a possible source of the syllable-final glottal stop.

\begin{tabular}{|c|c|c|c|}
\hline Opposition & Minimal pair & $P T B$ & Miji/Hruso \\
\hline /o?/ vs. /o:/ & $\begin{array}{l}\text { malò' 'penis' vs. } \\
\text { malo: '1. hard, } 2 . \\
\text { grandfather' }\end{array}$ & $\begin{array}{l}\text { PT *mrak PENIS; *s-ra } \\
\text { HARD/POOR }\end{array}$ & goyou (AA) 'hard' \\
\hline$/ u^{?} / \mathrm{v}$ & 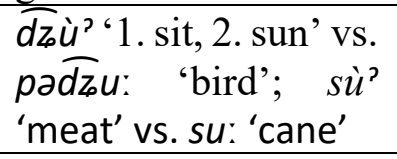 & $\begin{array}{ll}* \mathrm{~m}-(\mathrm{t} / \mathrm{d}) \mathrm{u}(\mathrm{y} / \mathrm{k}) & \text { SIT; } \\
* \text { daw OR } * \text { dow } & \text { BIRD; }\end{array}$ & $\begin{array}{l}\text { dzu? (AW) 'sit'; } \\
\text { dzo? (AW, } \mathrm{AE}) \\
\text { 'sun'; fitfun (AW), }\end{array}$ \\
\hline
\end{tabular}




\begin{tabular}{|c|c|c|c|}
\hline Opposition & Minimal pair & $P T B$ & Miji/Hruso \\
\hline & & $\begin{array}{l}\text { *sya-n MEAT; }^{*} \text { s-rwi(y) } \\
\text { CANE/CORD; }\end{array}$ & $\begin{array}{l}\text { sikijun (AE), sə (SA) } \\
\text { 'meat'; fu (SM, SA) } \\
\text { 'cane' }\end{array}$ \\
\hline /i/ vs. /i:/ & $\begin{array}{l}\text { gì 'cloth' vs. gi: } \\
\text { 'stand' }\end{array}$ & & $\begin{array}{l}\text { gijon, garon (AW, } \\
\mathrm{AE}) \text { 'stand' }\end{array}$ \\
\hline$/ \varepsilon^{?} /$ vs. / $\varepsilon: /$ & $\begin{array}{l}\operatorname{mar}(j) \dot{\varepsilon}^{\prime} \quad \text { 'leaf' } \\
\text { mare: 'near' }\end{array}$ & $\begin{array}{lll}\text { *rwak } & \text { LEAF; } & \text { *s-ney } \\
\text { NEAR } & & \end{array}$ & $\begin{array}{l}\text { ule? male? (AW) } \\
\text { 'leaf'; maro' (AE) } \\
\text { 'near' }\end{array}$ \\
\hline /a'/ vs. /a:/ & $\begin{array}{l}\text { ka } \overline{d z} \partial^{\prime} \text { 'thorn' vs. } \\
\overline{d z \partial} \text { : 'frog' }\end{array}$ & $\begin{array}{l}* \mathrm{~m}-(\mathrm{d}) \mathrm{z}(\mathrm{y}) \mathrm{u}(:) \mathrm{k} \\
\text { PIERCE/THORN }\end{array}$ & $\begin{array}{l}\text { gadzik (AW) } \\
\text { 'thorn'; dzou (AW, } \\
\text { AE) 'frog' }\end{array}$ \\
\hline$/ a^{2} /$ vs. /a:/ & $\begin{array}{l}\text { là' 'fireplace' vs. la: } \\
\text { 'sell' }\end{array}$ & $\begin{array}{l}*_{\text {g-rap }} \text { FIREPLACE; } \\
\text { (g/m/s)-lay } \\
\text { EXCHANGE } \\
\text { /BARTER }\end{array}$ & $\begin{array}{l}\text { dathle } \\
\text { 'fireplace' }\end{array}$ \\
\hline /e?/ vs. /e:/ & $\begin{array}{l}\text { rè? 'six' vs. ka:re: } \\
\text { '1DU' }\end{array}$ & $*$ d-k-ruk SIX & re? (AW) 'six' \\
\hline /ai?/ vs. /ai// & $\begin{array}{l}\text { ràì' 'grind, to crush' } \\
\text { vs. karai 'bow' }\end{array}$ & $\begin{array}{l}\text { *kri:t } \quad \text { GRIND; } \\
*(\mathrm{~g} / \mathrm{b} / \mathrm{m})-\mathrm{la}-\mathrm{y} \\
\text { BOW/ARROW }\end{array}$ & $\begin{array}{l}r i \quad(\mathrm{SM}) ; \quad r i \quad(\mathrm{SA}) \\
\text { 'grind'; } k^{h} \text { iri }(\mathrm{AA}, \\
\mathrm{AW}), \quad \text { geri gri } \\
\text { (AW), } k \partial r a^{i} \text { (AE) } \\
\text { 'bow' }\end{array}$ \\
\hline
\end{tabular}

Table 1. Examples of long vowel, level pitch vs. short vowel, falling pitch

A preliminary acoustic analysis indeed showed a significant difference in vowel length, pitch difference and spectral tilt. For the purpose of this paper, we analysed this difference as a difference of underlying open and closed syllables, rather than a difference in tone or vowel length. The comparative evidence in Table 1 suggests that the short-falling roots often derive from roots with consonantal coda.

\subsection{SYLLABLE AND WORD STRUCTURE}

The maximally permitted structure of the Bangru syllable is $\mathrm{C}_{\mathrm{i}}(\mathrm{G}) \mathrm{VC}_{\mathrm{f}}$. The minimal syllable consists of a vowel, though thus far only a few occurrences have been attested. Examples of each possible syllable structure include: $\mathrm{V}: \tilde{\varepsilon}:$ 'paddy

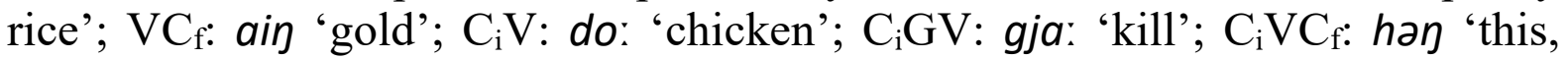
here'; $\mathrm{C}_{\mathrm{i}} \mathrm{GVC}_{\mathrm{f}}$ : mamjan 'name'.

Within syllable structure $\mathrm{C}_{\mathrm{i}} \mathrm{GV}$ and $\mathrm{C}_{\mathrm{i}} \mathrm{GVC}_{\mathrm{f}}$, the $\mathrm{C}_{\mathrm{i}}$ slot can be occupied by stops $/ \mathrm{k}, \mathrm{t}, \mathrm{p}, \mathrm{g}, \mathrm{d}, \mathrm{b} /$, the nasal $/ \mathrm{m} /$, the approximant $/ \mathrm{w} \sim \mathrm{v} /$, the fricative $/ \mathrm{s} /$ and the trill $/ \mathrm{r} /$. The $\mathrm{G}$ slot can be occupied by trill $/ \mathrm{r} /$ or glide $/ \mathrm{j} /$. The most common vowels following a consonant cluster are the open, central unrounded vowel /a/ and the 
open-mid, near-front, unrounded vowel $/ \varepsilon /$. In isolated cases, the open, close-mid back vowel /o/ after clusters $/ \mathrm{kj}, \mathrm{gj}, \mathrm{kr} /$, the schwa /a/ following clusters $/ \mathrm{rj}, \mathrm{mj}$, $\mathrm{kr} /$ and the back rounded vowel $/ \mathrm{u} /$ following the cluster $/ \mathrm{rj} /$ have been attested. In many speakers and in allegro speech, the consonant clusters $/ \mathrm{dj}, \mathrm{lj}, \mathrm{rj}, \mathrm{wj} \sim \mathrm{vj}$, $\mathrm{gj}, \mathrm{bj}, \mathrm{mj} / \mathrm{when}$ followed by vowel / $/$ / and the consonant clusters $/ \mathrm{wj}, \mathrm{lj}, \mathrm{sj}, \mathrm{rj}$, $\mathrm{mj} /$ when followed by vowel /a/ are often realised without glide and with long, open-mid, near-front unrounded vowel $/ \varepsilon: /$.

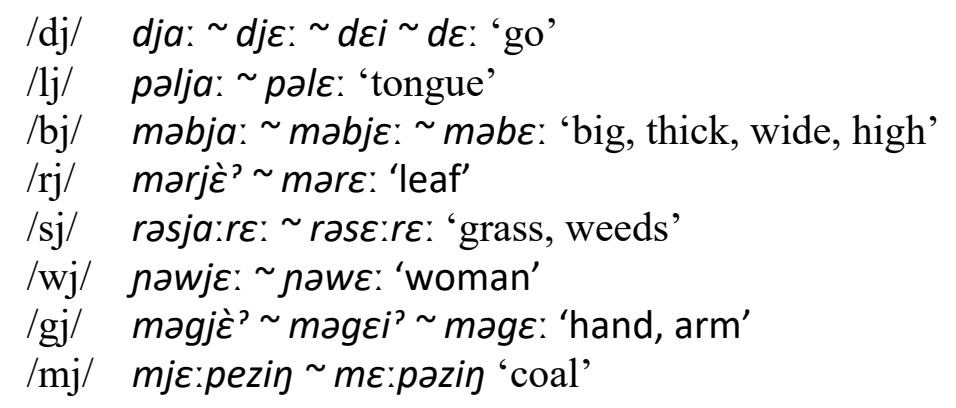

In the case of consonant clusters with the trill $/ \mathrm{r} /$, the reconstructed Proto-TibetoBurman forms commonly reflect complex initial consonant clusters $\left({ }^{*} \mathrm{CrV}\right)$. In Bangru, speakers will insert a schwa in careful speech (CorV), however, this schwa is again omitted in allegro speech $(\mathrm{CrV})$.

\section{/pr/ lapari: lapri: 'soft, smooth' cf. PTB *pryaw-k \\ /kr/ makaro: makro: 'straight' cf. PTB *t(r)wan}

The Bangru word, in particular the Bangru verb, can consist of several prefixes, suffixes and perhaps infixes, whose description would go beyond the scope of this paper. Unstressed, phonologically reduced syllables of the form $\mathrm{V}$ or $\mathrm{C}_{\mathrm{i}} \mathrm{V}$ are commonly aligned to the left of the fully formed stressed syllables of the form $\mathrm{C}_{\mathrm{i}}(\mathrm{G}) \mathrm{V}\left(\mathrm{C}_{\mathrm{f}}\right)$. The reduced syllables contain reduced vowels, i.e. a pre-glottalised back vowel /a/ or an epenthetic schwa/ə/. In the majority of cases, these are prefixes, which Bangru has largely preserved.

\begin{tabular}{|c|c|c|c|}
\hline Prefix & Function & Examples & $P T B / O C$ \\
\hline ma- & $\begin{array}{l}\text { animated/human prefix; } \\
\text { e.g. kinship: 'the other's' } \\
\text { and body parts: } \\
\text { 'inalienable part of self' }\end{array}$ & $\begin{array}{l}\text { maje: 'someone else's } \\
\text { mother'; momua } \\
\text { 'someone's elder sister'; } \\
\text { malagu: } \\
\text { intestines'; 'guts, malawja: } \\
\text { 'tail' }\end{array}$ & $\begin{array}{l}\text { OC *mi 'animated/human' } \\
\text { (Baxter and Sagart 2014: } \\
55) \text {, PTB } *_{m-} \text { '3rd person } \\
\text { poss.' }\end{array}$ \\
\hline & adj. & $\begin{array}{l}\text { mapan 'long'; mawa: } \\
\text { 'round' }\end{array}$ & $\begin{array}{l}\text { OC *mə- for volitional } \\
\text { verbs (Baxter and Sagart } \\
\text { 2014: 55) }\end{array}$ \\
\hline & adv. and proposition & 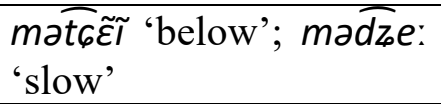 & -do- \\
\hline
\end{tabular}




\begin{tabular}{|c|c|c|c|}
\hline Prefix & Function & Examples & $P T B / O C$ \\
\hline \multirow[t]{2}{*}{$\begin{array}{l}\text { ga- } \\
\text { /go- }\end{array}$} & part of head & 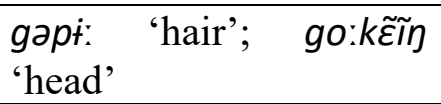 & $*_{m} / s-g a w$ HEAD \\
\hline & related to trees & $\begin{array}{l}\text { gapja: 'bark'; gane: } \\
\text { 'tree' }\end{array}$ & PH go 'wood' \\
\hline \multirow[t]{2}{*}{$a-$} & kinship: 'the own' & $\begin{array}{l}\text { ane: 'own mother'; } a m a: \\
\text { 'own elder sister' }\end{array}$ & *?a- 'kinship' \\
\hline & adj. & $\begin{array}{l}\text { amam 'fat, stout'; aran } \\
\text { 'far' }\end{array}$ & - \\
\hline \multirow[t]{2}{*}{ la- } & adj. & $\begin{array}{l}\text { latõ: 'light'; lapri: 'soft, } \\
\text { smooth' }\end{array}$ & - \\
\hline & natural elements & $\begin{array}{l}\text { alo: labai 'grandfather } \\
\text { moon'; lat } \bar{t} \text { :' 'star' }\end{array}$ & - \\
\hline sa- & animals & $\begin{array}{l}\text { sakja: 'animal'; satढ̄̃ } \\
\text { 'bear' }\end{array}$ & ${ }^{*} s y a-n$ MEAT \\
\hline
\end{tabular}

Table 2. Bangru prefixes.

Miji and Hruso have preserved fewer prefixes. Eastern and Western Miji have an adjective prefix $\langle m a->$, except for the Dibbin variety, which has an adjective prefix $<a->$. Eastern and Western Miji also have the $<a->$ and $<m a->$ prefixes for kinship terms, but do not appear to make the 'own' versus 'other' distinction, i.e. the distinction between 'my own mother' versus 'someone else's mother'. Hruso body parts are commonly prefixed with any of the simple vowels.

\section{GENETIC CLASSIFICATION OF BANGRU}

Based on lexical comparison, Anderson (2014) adduced for Bangru, Miji and Hruso what Shafer (1947) earlier proposed for Miji and Hruso, namely that they form a coherent subgroup of the Tibeto-Burman language family. In this section, we will present additional evidence for this. In our Bangru data, we found reconstructed proto-forms for around $50 \%$ of the 360 lexical entries appearing in both our data set and the STEDT database ${ }^{5}$. This is a first indication that Bangru can be considered as Tibeto-Burman language. Bangru's affiliation with the other Hrusish languages Miji and Hruso can also be illustrated through the shared pronominal and numeral paradigms in Table 3 and 4. Regular sound correspondences between the reconstructed Proto-Tibeto-Burman roots and modern Bangru, Miji and Hruso reflexes given below further lend evidence to the affiliation of these languages with the other Tibeto-Burman languages. The STEDT database references and glosses of reconstructed Proto-Tibeto-Burman

\footnotetext{
${ }^{5}$ More detailed and complete overviews, including original sound files, will be provided upon request. A tilde $(\sim)$ in this and following tables indicates both distinctly transcribed entries as well as variant forms from secondary sources. Unless mentioned otherwise, Eastern and Western Miji and Hruso forms are from Abraham et al. (2005).
} 
roots can be found in appendix $\mathrm{C}$, a guide to the used abbreviations and glosses can be found in appendix A.

\begin{tabular}{|c|c|c|c|c|c|}
\hline Gloss & $P T B$ & Bangru & E. Miji & W. Miji & Hruso \\
\hline $1 \mathrm{SG}$ & *na-y & no: & nijan & 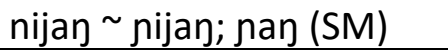 & no \\
\hline $2 \mathrm{SG}$ & *nan & ni: ni: & ni & ni & $b^{h} a$ \\
\hline $3 \mathrm{SG}$ & - & petढ़i & $a^{i}$ & $i \sim p^{h} \overline{e t \int u}$ & Pi \\
\hline $1 \mathrm{PL}^{6}$ & *nəy & kani: $\sim$ kani: $^{7}$ & $\begin{array}{l}\text { ani } \sim \\
\text { animihõn }\end{array}$ & ani anijaahun & ni; ani (SA) \\
\hline $2 \mathrm{PL}$ & - & ni:; $\overline{d z e}:$ & $\begin{array}{l}\text { dzimihõn } \\
\sim \text { dzei }\end{array}$ & dzi dzimə]un ina & d30 \\
\hline 3PL & - & $\begin{array}{l}\text { dže:(mələy/ } \\
\text { kadł) }\end{array}$ & $a^{i} r a$ & $\begin{array}{l}\text { inamehijan } \sim p^{\text {h}} \text { aina } \sim \\
\text { airaahun }\end{array}$ & na \\
\hline this & - & hay & & hunji hunlo hãnai & $\begin{array}{l}\text { heji; hunlo } \\
\text { (SA) }\end{array}$ \\
\hline $\begin{array}{l}\text { that (same } \\
\text { level) }\end{array}$ & - & $p \varepsilon: \bar{t} \epsilon(i)$ & & 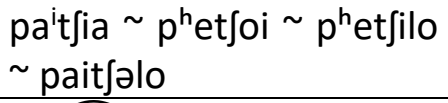 & - \\
\hline $\begin{array}{l}\text { that (higher } \\
\text { level) }\end{array}$ & - & to: $\bar{t} \int(i)$ & & thantsu (SM) & - \\
\hline $\begin{array}{l}\text { that (lower } \\
\text { level) }\end{array}$ & - & pu:tढ $(i)$ & & $\mathrm{p}^{\mathrm{h}} \widehat{\mathrm{utsu}}$ (SM) & - \\
\hline
\end{tabular}

Table 3. Comparison of personal pronouns and demonstrative pronouns.

\begin{tabular}{|c|c|c|c|c|c|c|}
\hline Gloss & $P T B$ & Bangru & E. Miji & & W. Miji & Hruso \\
\hline one & PT *kon & ’akã & $\begin{array}{l}a k^{h} i j o \\
a k^{h} o w a \\
a \eta\end{array}$ & $\sim$ & $\begin{array}{l}\text { ak }{ }^{h} \text { ijo } \sim a k^{h} e ? \sim a k^{h} o \sim \\
\text { atro }\end{array}$ & $\mathrm{Pa}$ \\
\hline two & $*(\mathrm{~g} / \mathrm{s})-\mathrm{ni}-\mathrm{s}$ & kərعi & $\begin{array}{l}k^{\mathrm{h}} \text { erə } \\
\text { kəran }\end{array}$ & $\sim$ & geni $\sim$ gərin & kfə \\
\hline three & *sum & kət $\tilde{\varepsilon} \tilde{I}$ & $\mathrm{k}^{\mathrm{h}} \partial t \partial \mathrm{m}$ & & gətən kh & dzว \\
\hline four & *b-lәу & puruwẼ Ĩ & pəle ${ }^{i}$ & & bli bəle ${ }^{i}$ & psăxi $\sim p^{h_{i r i}}(S A)$ \\
\hline five & $* I / b-\eta a$ & рәрu: & punu & & bunu boju pugu & pom; pum (SA) \\
\hline six & $*^{*} \mathrm{~d}-\mathrm{k}$-ruk & rè? & $\mathrm{ra}(\mathrm{m})$ & & $\operatorname{re}(?)$ & $x e ;$ rije (SA) \\
\hline seven & - & məwài? & mija & & mija(k) & mıo; mrjo (SM) \\
\hline eight & *b-r-gyat & səgài? & 6əgə & & səgə(i) tfigə & $\int \partial g d z \partial$ \\
\hline nine & - & sətəク & grtan & & sətən & sțə; st ${ }^{\mathrm{h}} \partial$ (SA) \\
\hline ten & PT * rjun & ran & lən & & tən lan & $x \partial ; y \partial \sim$ rə (SA) \\
\hline hundred & PT *lun & lə刀 & pəlon & & bəlun blun & фоха; $p^{h} u y u$ (SA) \\
\hline
\end{tabular}

Table 4. Comparison of numerals.

\footnotetext{
${ }^{6}$ Bangru does not have distinctive forms for first person plural inclusive or exclusive pronouns. The Bangru first person dual pronoun is kare: and the second person dual pronoun is $\overline{d z}$ are:.

${ }^{7}$ Perhaps reflecting egophoric first person pronoun PTB *ka-, cf. PKC kay $\Varangle$ kay-ma? and various other languages of the extended Eastern Himalayas.
} 
The correspondences between the pronouns and numerals in these languages can not be coincidental and are suggestive of a close historical relationship, be it a genetic relationship or one of intense contact. In addition to the lexical cognates and shared pronoun and numeral paradigms, we have identified 35 sound correspondences between the reconstructed Proto-Tibeto-Burman forms and the Bangru forms. In a reasonable number of cases, these sound correspondences are shared by Miji and Hruso. Not too much weight has been given to exceptions to the correspondences in the Western and Eastern Miji and Hruso cognates, because of having to rely on secondary data with transcriptions of variable reliability. These irregular correspondences are given between parentheses. The main correspondences for consonants we established are summarised in Table 5 and supportive sets are provided in detail in sections 4.1-4.7. Similarly, the supportive sets for the vowel correspondences are summarised in Table 32 and illustrated in section 4.8. Realisations after a slash represent equally frequent realisations, realisations between parentheses are marginal, and the forelast column presents a count of supporting sets.

\begin{tabular}{|c|c|c|c|c|c|c|}
\hline$P T B$ & Bangru & Eastern Miji & Western Miji & Hruso & $\#$ & Table \\
\hline *s- & $t-$ & t- & $t^{h}-\left(t \int-\right)$ & d3- & 6 & 6 \\
\hline${ }^{*}$ g-/*b- (pref.) & $k-/ p-$ & $k-/ p-$ & g-/b- & $k\left({ }^{h}\right)-/ p\left(^{h}\right)-$ & $2 / 2$ & 9 \\
\hline$*_{\mathrm{t}-}$ & t- & $t-$ & $t^{h_{-}}$ & $t^{h}-(d z-)$ & 4 & 10 \\
\hline$* d-$ & $d-\left(\widetilde{d z}-/ t_{6}-\right)$ & d- $\left(d_{3}-/ t f-\right)$ & d- (dz-/tf-) & d- $\left(d z-/ t \int-\right)$ & 11 & 11 \\
\hline${ }^{*} k(G)-$ & k- & $k-/ k^{h}-$ & $k^{h}-$ & $k J-$ & 7 & 12 \\
\hline$* g(G)-$ & g- & g- & g- & gd3- & 2 & 13 \\
\hline${ }^{* b}-/{ }^{*} \mathrm{p}-$ & $b-/ p-$ & $b-/ p\left(^{h}\right)-$ & $b-/ b-$ & $b-(d z-) / p^{h}-$ & $3 / 1$ & 14 \\
\hline$*-\mathrm{k}$ & $-v^{2}(-\varnothing)$ & $-\varnothing$ & $-\varnothing(-k /-?)$ & $-\varnothing$ & 9 & 15 \\
\hline$*_{-t}$ & $-v^{2}(-\varnothing)$ & $-\varnothing$ & $-\varnothing(-P)$ & $-\varnothing$ & 5 & 16 \\
\hline$*-p$ & $-\varnothing\left(-v^{2}\right)$ & $-\varnothing /-m$ & $-\varnothing(-m)$ & $-\varnothing$ & 3 & 17 \\
\hline$* m-$ & m- & m- & m- & $\mathrm{m}-$ & 5 & 18 \\
\hline${ }^{*} n-/{ }^{*} n-$ & $r-$ & $r-$ & $r-/ n-$ & $n-(n-)$ & 6 & 19 \\
\hline$*_{n-/ * n-}$ & $n-/ n-$ & $n-/ n-$ & $n-/ n-$ & $n-/ n-$ & 5 & 20 \\
\hline$*_{-m}$ & $-\varnothing$ & $-\varnothing /-m$ & $-\varnothing /-n$ & $-\varnothing$ & 4 & 21 \\
\hline$* \mid-$ & $1-$ & $1-$ & $1-(t-)$ & $1-$ & 7 & 22 \\
\hline$* \mid-$ & $r-$ & $1-(r-)$ & $1-(r-)$ & $r-$ & 3 & 23 \\
\hline$* \mid-$ & I- & j- (I-) & $j-\left(\int-/ d z^{-}\right)$ & I- & 4 & 24 \\
\hline$*_{r-}$ & $r-$ & $r-$ & $r-$ & $r-/ x-$ & 6 & 25 \\
\hline$*_{r-}$ & $r-$ & $1-/ r-$ & I- & $r-/ x-$ & 3 & 26 \\
\hline$*(t) s(y)-$ & $\widehat{t 6-}$ & tf- & tf- & $\mathrm{t}$ - & 3 & 27 \\
\hline$*(d) z(y)-$ & $\widehat{d t_{0}}-\widehat{t \varphi_{6}}-$ & dz- (t t -$)$ & d3-/t $\int-$ & $\mathrm{t} \int-\left(\mathrm{d} z-/ \int-\right)$ & 4 & 28 \\
\hline
\end{tabular}

Table 5. Summary of sound correspondences (consonants). 


\subsection{PROTO-TIBETO-BURMAN FRICATIVE *S-TO PLOSIVE T-.}

Shafer (1947: 185) was the first to describe one of the defining phonological innovations that Bangru, Miji and Hruso share with the 'Kamarupan' languages (Matisoff 2003: 31-32), including the Kuki-Chin languages (VanBik 2009: 16), Bodo-Garo, Karbi and Tangkhulic. This is the change from Proto-Tibeto-Burman $*_{s-}$ to voiceless alveolar plosive $t-/ t^{h}$.

\begin{tabular}{|c|c|c|c|c|c|}
\hline Gloss & $\begin{array}{l}P T B \\
*_{S-}\end{array}$ & $\begin{array}{l}\text { Bangru } \\
t-\end{array}$ & $\begin{array}{l}\text { E. Miji } \\
t-\end{array}$ & $\begin{array}{l}W . M i j i \\
t^{h}-/ t-\end{array}$ & $\begin{array}{l}\text { Hruso } \\
d_{3-}\end{array}$ \\
\hline liver & $\begin{array}{l}* \text { m-sin; PKC *thin: PTk } \\
t^{\text {hin }}\end{array}$ & 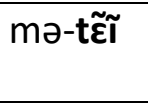 & - & mə-thon (SM) & ě-djǔ (AS) \\
\hline die & $\begin{array}{l}\text { *səy; PKC *thii-I, thi?-II; } \\
\text { PBG *thui }{ }^{1} ; \text { PTk t }{ }^{\text {hi }}\end{array}$ & tai & tei-na & $t \int i$ & $\begin{array}{l}\text { dzu-dzo dza/i } \\
\text { (SA) }\end{array}$ \\
\hline seed & ${ }^{*}$ sey; PTk *tha; PKC * $\theta$ an & 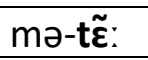 & $t^{i}-10^{i}$ & $t^{h} \mathbf{e}-l i \sim t^{h} e-d z 0$ & 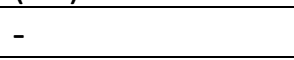 \\
\hline fruit & $\begin{array}{l}* \text { sey; PKC *thay; PBG } \\
* \text { thai }^{2} ; \text { PTk } * t^{\text {hej }} \text { ej }\end{array}$ & mə-tẽ: & gu-tan & $u-t^{h} e n$ & $\int \breve{-d z e}$ \\
\hline three & $\begin{array}{l}* \text { sum; PBG *tham }{ }^{2} ; \text { PKC } \\
* \text { thum; PTk *thum }\end{array}$ & 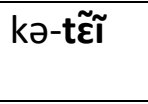 & $k^{h} \partial-t \partial m$ & $\begin{array}{l}k^{h} \text { ə-təm } \sim \text { kə- } \\
t^{\text {h}} \text { ən ( gə-tən) }\end{array}$ & d3ə \\
\hline who & $*_{\text {su; }}$ PKC *tuu & ti:-wo & təo & $t \int i u \sim t \int^{h} u$ & d3u \\
\hline
\end{tabular}

Table 6. Proto-Tibeto-Burman ${ }^{*}{ }_{-}>t-$.

The innovation Proto-Tibeto-Burman $*_{s-}>t$ - is shared by Bangru, Miji and Hruso. Bangru and Eastern Miji have well preserved this initial innovation, with reflexes of plosive $t$ - in all cases. Counterexamples include those lexemes where the $*_{\mathrm{s}}$ - $>\mathrm{t}$ - development preceded simplification of Proto-Tibeto-Burman consonant clusters with initial $*_{s}$. The following examples show that this initial /s/ was preserved in all the Hrusish languages, albeit with subsequent palatalisation in a number of instances.

\begin{tabular}{|c|c|c|c|c|}
\hline Gloss & $\begin{array}{l}P T B \\
*_{s} G-\end{array}$ & $\begin{array}{l}\text { Bangru } \\
s-\end{array}$ & $\begin{array}{l}M i j i \\
s-\left(3-/ \int-\right)\end{array}$ & $\begin{array}{l}\text { Hruso } \\
s-/ \int-/ t s-\end{array}$ \\
\hline louse & *s(y)ar & sà? & - & fə (SA) \\
\hline meat & ${ }^{*}$ sya-n; PKC *s ${ }^{h}$ aa & sù? Gù? & 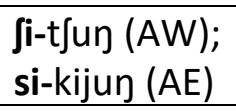 & tso (SA) \\
\hline grandmother & $*_{\mathrm{sru}}(\mathrm{w})$ & a-se: & a-3ui (SM) & - \\
\hline iron & *syam & SO: & $\operatorname{sen}(\mathrm{SM})$ & so (SA) \\
\hline flow & $*_{\text {sywar } \}$ *g/b-sywa-n/t & sa: & - & - \\
\hline
\end{tabular}

Table 7: Counterexamples to $*_{s-}>t-:$ initial clusters 


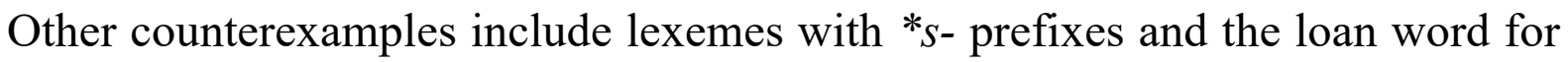
'breath'.

\begin{tabular}{|c|c|c|c|c|}
\hline Gloss & $\begin{array}{l}P T B \\
* s-(\text { prefix) }\end{array}$ & $\begin{array}{l}\text { Bangru } \\
\text { s- }\end{array}$ & $\begin{array}{l}\text { Miji } \\
\int-\left(t^{h_{-}}\right)\end{array}$ & $\begin{array}{l}\text { Hruso } \\
\int-(s-)\end{array}$ \\
\hline breath & $\begin{array}{l}\text { *N-sak; PCN *sak; Proto-Western Tani } \\
\text { *sak BREATH(v); OC *sək }\end{array}$ & sò? & $\begin{array}{l}\text { di-tth } \\
(S M)\end{array}$ & - \\
\hline dead body & ${ }^{*}$ s-man ; PT *si-man & so-mo: & - & - \\
\hline cane; rope & $\begin{array}{l}\text { *s-rwi(y); PKC *ruy } \Varangle \text { hruy; PCN *a-(h)rəj; } \\
\text { PT *son; Bengni u-šo: (Sun 1993: 93) }\end{array}$ & $\begin{array}{l}\text { su: } \\
\text { 6u: }\end{array}$ & Ju (SM) & fu (SA) \\
\hline live, grow up & 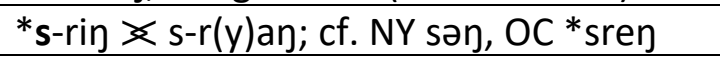 & səy & Jən (SM) & əЈə (SA) \\
\hline otter & *s-ram & sẽ: & - & size (SA) \\
\hline
\end{tabular}

Table 8: Counterexamples for $*_{s-}>t$-: s-prefixes.

\subsection{RETENTION OF SYLLABLE-INITIAL PLOSIVES.}

Proto-Tibeto-Burman syllable-initial plosives were found to be preserved and have regular reflexes in the Hrusish languages. First of all, in prefixes, the ProtoTibeto-Burman $* b$ - and $* g$ - were devoiced in Hruso, Bangru and the Eastern Miji varieties, but not in Western Miji.

\begin{tabular}{|c|c|c|c|c|c|}
\hline Gloss & $\begin{array}{l}P T B \\
*_{b-/ *_{g}}\end{array}$ & $\begin{array}{l}\text { Bangru } \\
p-/ k-\end{array}$ & $\begin{array}{l}\text { E. Miji } \\
p-/ k^{-}\end{array}$ & $\begin{array}{l}W . M i j i \\
b-/ g-\end{array}$ & $\begin{array}{l}\text { Hruso } \\
p\left({ }^{h}\right)-/ k\left(^{h}\right)-\end{array}$ \\
\hline four & *b-ləy & 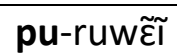 & рә-le $e^{i}$ & bli bə-le $e^{i}$ & psə̆-xi p $^{\text {hi-ri }}(S A)$ \\
\hline five & $* 1 / \mathbf{b}-$ пa & pə-nu: & pu-nu & $\begin{array}{l}\text { bo-pu bu- } \\
\text { pu ( pu-gu) }\end{array}$ & p-om; p-um (SA) \\
\hline two & $*(\mathrm{~g} / \mathrm{s})-\mathrm{ni}-\mathrm{s}$ & kə-rعi & kə-ran k he-rə & $\begin{array}{l}\text { ge-ni gə- } \\
\text { rin }\end{array}$ & 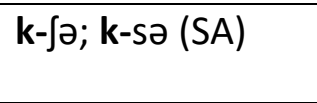 \\
\hline bow & $*(\mathbf{g} / \mathrm{b} / \mathrm{m})-\mathrm{la}-\mathrm{y}$ & kə-ràì? & kə-rai & $\begin{array}{l}\text { gə-ri gri ( } \\
\text { k }^{\text {hi-ri) }}\end{array}$ & $k^{h} \mathbf{i}-r i$ \\
\hline
\end{tabular}

Table 9. Reflexes of PTB prefixes ${ }^{*} g$-/b-in prefixes.

Other than in prefixes, Proto-Tibeto-Burman $* b-/ * g-/ * d$ - tend to correspond with Hrusish voiced plosives $b$ - $/ g-/ d$ - . Proto-Tibeto-Burman ${ }^{*} p-/ *^{*}-/ *^{*}$ - tend to correspond with Hrusish unvoiced $p-/ k-/ t$-, often aspirated in Western Miji and Hruso. Affrication of plosives is common in the Hrusish languages as a secondary development. Major exceptions include voicing inconsistencies in reflexes of Proto-Tibeto-Burman *(t/d)ak WEAVE: Bangru tढai, Eastern Miji tjom-na ga ${ }^{i}-$ tfom, Western Miji (zan/gra-)trin $\sim 62 n \sim t^{h} r a n$, Hruso $t^{h} i$-jo, all perhaps under influence of Tani, cf. Proto-Tani *cum (provisional) and the reflexes for ProtoTibeto-Burman *t(u/i)k FROG Bangru dża:, Miji dzou, Hruso Je-dza. 


\begin{tabular}{|c|c|c|c|c|c|}
\hline Gloss & $\begin{array}{l}P T B \\
*_{t-}\end{array}$ & $\begin{array}{l}\text { Bangru } \\
\text { t- }\end{array}$ & $\begin{array}{l}E . M i j i \\
t-\end{array}$ & $\begin{array}{l}W . M i j i \\
t^{h_{-}}\end{array}$ & $\begin{array}{l}\text { Hruso } \\
t^{h}-\left(d z_{-}\right)\end{array}$ \\
\hline drink & $* \mathrm{~N} / \mathrm{s}-\mathrm{tu \eta}$ & ton $\sim$ tun & $\begin{array}{l}\text { ton-na } \\
\left(\sim \mathrm{t}^{\mathrm{h}} \text { on }\right)\end{array}$ & $\mathbf{t}^{\mathrm{h}}$ on(-me) & $\mathbf{t}^{\mathrm{h}} \mathbf{u}-\mathrm{d}$ 30 (AA) \\
\hline bite & $*_{\text {twak }}$ & to: & ta(P-na) & $t^{\text {hai }} \sim t^{h} a ?$ & $\mathbf{t}^{\mathrm{h}} \mathbf{o - d}$-do (AA) \\
\hline tooth & $*_{\text {twan }^{8}}$ & mə-tu: & to & $\mathbf{t}^{\mathbf{h}} \mathbf{u}(\sim \mathrm{tu})$ & Pe-t $\mathbf{t}^{\mathbf{h}} \mathbf{u}(\mathrm{AA})$ \\
\hline neg. imp. & $*(\mathrm{t} / \mathrm{d}) \mathrm{a}$ & tu- & - & $t^{h} a-(S M)$ & dzu- (SA) \\
\hline
\end{tabular}

Table 10. Reflexes of PTB *t-.

\begin{tabular}{|c|c|c|c|c|c|}
\hline Gloss & $\begin{array}{l}P T B \\
* d-\end{array}$ & $\begin{array}{l}\text { Bangru } \\
d-/ \overline{d z_{0}}-(\widehat{t \epsilon}-)\end{array}$ & $\begin{array}{l}\text { E. Miji } \\
d-/ d z^{-}(t-)\end{array}$ & $\begin{array}{l}\text { W. Miji } \\
d-/ d z-\left(t f^{-}\right)\end{array}$ & $\begin{array}{l}\text { Hruso } \\
d-/ d z-\left(t \int_{-}\right)\end{array}$ \\
\hline chicken & $*$ daw OR *dow & do: & do $^{u}$ & $\begin{array}{l}\text { dok do? } \\
\text { dup }\end{array}$ & d3uo \\
\hline $\begin{array}{l}\text { big (thick, } \\
\text { wide) }\end{array}$ & $*(\mathrm{t} / \mathrm{d})$ ow-n & mə-dua & $\begin{array}{l}\text { mə-do } \\
\text { mə-də-bu }\end{array}$ & $\begin{array}{l}\text { mə-do a- } \\
\text { do }\end{array}$ & $\begin{array}{l}\text { djo; } \\
\text { (SA) }\end{array}$ \\
\hline have; exist & $* \mathrm{du}$ & du: & - & du (SM) & du (SA) \\
\hline make; do & $*$ day & $\mathrm{da} \sim \mathrm{d} \varepsilon:$ & - & - & da-d3o \\
\hline spear & $\begin{array}{l}*_{\mathrm{m}} \text {-dun } \\
\text { day }\end{array}$ & 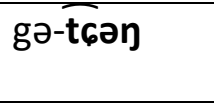 & dzon/? & dzun & $t \int u-d z u$ \\
\hline sit, stay & $*_{\mathrm{m}}-(\mathrm{t} / \mathrm{d}) \mathrm{u}(\mathrm{y} / \mathrm{k})$ & dzùt? & - & $\begin{array}{l}\text { deyra-dzun } \\
\sim \text { dzup }\end{array}$ & - \\
\hline bear & $*$ d-wam & sə-tढ़õ: & fi-t]an & $\begin{array}{l}\text { fi-t]an } \sim \int \mathrm{i}- \\
\text { stan }\end{array}$ & $\int \breve{\partial-t \int o}$ \\
\hline bird & $*$ daw OR *dow & 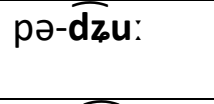 & bə-dzi & $\begin{array}{l}\text { bə-dzə } \\
\text { bu-dzu? }\end{array}$ & - \\
\hline body & $* \mathrm{du}$ & mə-dż̀े? & - & - & - \\
\hline burn & $*$ duk & dzo: & - & - & - \\
\hline child & $*_{\mathrm{m}}-(\mathrm{t} / \mathrm{d}) \mathrm{u}$ & mə-dzu: & - & - & - \\
\hline
\end{tabular}

Table 11. Reflexes of PTB *d-.

Proto-Tibeto-Burman *t-has as regular reflexes Bangru and Miji $t$ - $/ t^{h}$ - and Hruso $t^{h}$-. The fact that no merger between reflexes of Proto-Tibeto-Burman $*_{s-}$ and $*_{t}$ has taken place in Hruso indicates that at the Proto-Hrusish level, the Proto-

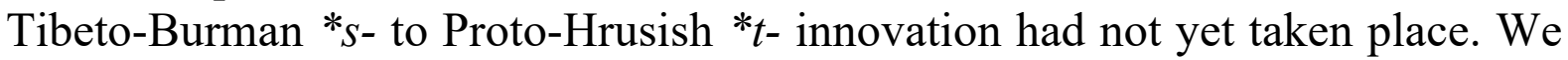
hence postulate that the Proto-Hrusish reflex of Proto-Tibeto-Burman *s- was an intermediate form, perhaps $* \vartheta$-, which then became $* t$ - in Proto-Miji-Bangru, giving the reflexes $t$-/ $/ t^{h}$ - in the modern languages, and $* t->(* t f-\sim) * d z$ - in Proto-

\footnotetext{
${ }^{8}$ As pointed out by Mr. Yeshy T. Sotrug, these forms might also be related to PTB \#632 *s/pwa through the $*_{s-}>t$ - change, see also Karen Taunthu tana 'tooth' (Benedict 1972: 137). The widespread occurance of roots cognates to *twan in the Kho-Bwa languages, the s-prefix in *s/p-wa and comparative evidence from PKC *haa, PTk *ha and PCN *p-hwa do not readily support this hypothesis.
} 
Hruso, giving the reflex $d z$ - in modern Hruso. The reflexes of Proto-TibetoBurman ${ }^{*}$ - in other language groups of Northeast India, such as $t-/ t^{h}$ - in KukiChin, Bodo-Garo, Central Naga and Tangkhulic, $\varnothing$ - in the Kho-Bwa languages, variation between $\varnothing$-, $s$-, $h$ - and $h$ - in Tani and the Mijuish languages and $s$ - in

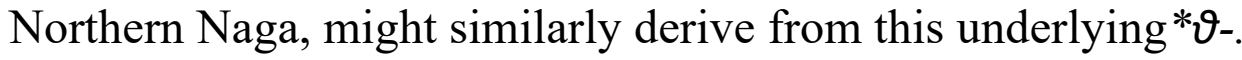

There are also a number of regular reflexes of the Proto-Tibeto-Burman syllableinitial $* k$ - and ${ }^{*} g$-.

\begin{tabular}{|c|c|c|c|c|c|}
\hline Gloss & $\begin{array}{l}P T B \\
* k(G)-\end{array}$ & $\begin{array}{l}\text { Bangru } \\
\text { k- }\end{array}$ & $\begin{array}{l}\text { E. Miji } \\
k-/ k^{h}-\end{array}$ & $\begin{array}{l}W . M i j i \\
k^{h_{-}}\end{array}$ & $\begin{array}{l}\text { Hruso } \\
\text { kf- (k-) }\end{array}$ \\
\hline $\begin{array}{l}\text { elder } \\
\text { brother }\end{array}$ & $\begin{array}{l}*(\mathrm{~g} / \mathrm{k}) \partial \mathrm{w}- \\
\mathrm{n}\end{array}$ & a-ko: & kə-voi & $\begin{array}{l}\text { a-kho-vo kho-vo? ( a- } \\
\text { kə-vo) }\end{array}$ & - \\
\hline pillow & $\begin{array}{l}*_{\mathrm{m}}- \\
\mathrm{k}(\mathrm{u} / \mathrm{i}) \mathrm{m}\end{array}$ & 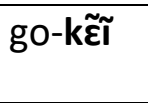 & go-kəm & (nu/wo)-k ${ }^{h}$ ən u-k $u m$ & di-kJo \\
\hline steal & *r-kəw & lo-kə: & 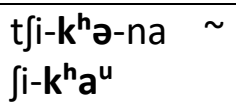 & $t \int i / t \int \partial-k^{h} \partial / k^{h} u$ & $k \int a-t \int u d z o$ \\
\hline cry & ${ }^{*} \mathrm{krap}$ & k(j)e: & krəm-na & krəm-na $\mathbf{k}^{\text {hə̆ }}$ & kJo \\
\hline smoke (n.) & ${ }^{*} \mathrm{k} \partial \mathrm{w}-\mathrm{n} / \mathrm{t}$ & me-kə: & mai-khon & mai-k $\mathbf{k}^{\mathrm{h}}$ & mu-kJə \\
\hline sew & *krwi(y) & kàì? & 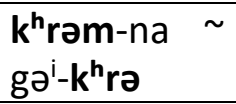 & $k^{h} r \partial \sim k^{h} r i$ & $k \int i-j o$ \\
\hline bitter & $\begin{array}{l}* \mathrm{~b}-\mathrm{ka}- \\
(\mathrm{n} / \mathrm{m} / \mathrm{y})\end{array}$ & kə-kjo: & $m ə-k^{h} \mathbf{u}$ & $m ə-k^{h} \mathbf{u} \sim a-k^{h} \mathbf{u}$ & kam-Jo \\
\hline
\end{tabular}

Table 12. Reflexes of PTB *k-

\begin{tabular}{llllll}
\hline Gloss & PTB & Bangru & E. Miji & W. Miji & Hruso \\
& ${ }^{g}(G)-$ & $g-$ & $g-$ & $g-$ & gdz- \\
\hline eight & $*_{\text {b-r-gyat }}$ & sə-gài & cə-gə & tfi-gə sə-gə(i) & Jə-gdzə \\
\hline mountain & *s-gay $^{\text {gan }}$ & - & - & - \\
\hline
\end{tabular}

Table 13. Reflexes of PTB * $g$ -

Like with Proto-Tibeto-Burman *g-, there are relatively few reflexes of ProtoTibeto-Burman $* p$ - and $* b$-.

\begin{tabular}{|c|c|c|c|c|c|}
\hline Gloss & $\begin{array}{l}P T B \\
* b-; * p(G)-\end{array}$ & $\begin{array}{l}\text { Bangru } \\
b-; p-\end{array}$ & $\begin{array}{l}\text { E. Miji } \\
b-; p-/ p^{h_{-}}\end{array}$ & $\begin{array}{l}W . M i j i \\
b-; b-\end{array}$ & $\begin{array}{l}\text { Hruso } \\
b-(d z-) ; p^{h_{-}}\end{array}$ \\
\hline insect & *bow & bə:-lon & bi-lo bi-lun & bi-lun & bŭ-I:u; bi-lu (SA) \\
\hline snake & *bow & bə: & bo ${ }^{u}$ & $\mathbf{b}^{\mathbf{h}} \mathbf{u} \sim$ na-bəu & by \\
\hline give & $*_{\text {s-bəy-n } / \mathrm{k}}$ & bai & $\mathbf{b e}^{\mathrm{i}}-\mathrm{na} \sim \mathbf{b a}^{\mathrm{i}}$ & bi $\sim p^{h} e-b i \sim ~ b i p-n e$ & dzi-dzo (SA) \\
\hline axe & $*_{r}-p^{w} a$ & pu-rudżin & pə̆-lə phĕ-lu & b-lu bu-lu & $p^{\text {h }}$ o-dze \\
\hline
\end{tabular}

Table 14. Reflexes of PTB * ${ }^{*}$-/b-. 


\subsection{LOSS OF SYLLABLE-FINAL PLOSIVES.}

Syllable-finally, there are few if any occurrences of plosives $/ \mathrm{k}, \mathrm{p}, \mathrm{t} /$. The quality of the vowels preceding lost syllable-finals varies between the languages and their varieties. Western Miji has the most conservative retention of syllable-final plosive $/ \mathrm{k} /$.

\begin{tabular}{|c|c|c|c|c|c|}
\hline Gloss & $\begin{array}{l}P T B \\
*_{-k}\end{array}$ & $\begin{array}{l}\text { Bangru } \\
-v^{2}(-\varnothing)\end{array}$ & $\begin{array}{l}E . M i j i \\
-\varnothing\end{array}$ & $\begin{array}{l}W . M i j i \\
-\varnothing(-? /-k)\end{array}$ & $\begin{array}{l}\text { Hruso } \\
-\varnothing\end{array}$ \\
\hline thorn & ${ }^{*} \mathrm{~m}-(\mathrm{d}) \mathrm{z}(\mathrm{y}) \mathrm{u}(:) \mathrm{k}$ & 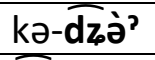 & $k^{h} \partial-z a u$ & gə-dzik & ku-tsu (AA) \\
\hline sit, stay & ${ }^{*} m-(t / d) u(\eta / k)$ & džù? & - & $\begin{array}{l}\text { deyra-dzun } \\
\text { dzu? }\end{array}$ & - \\
\hline eye & ${ }^{*}$ s-myak $\$ s-mik & me-jà? & mẽ & mər $\sim$ mə-ri $\sim$ me? & Pi-nii ${ }^{9}$ \\
\hline itch & $*_{\mathrm{m} \text {-tsik }}$ & kə-dzù? & - & gu-dzu (SM) & sə-dzu (SA) \\
\hline leaf & *rwak & mə-r'̀? & - & mə-le u-le? & $\begin{array}{l}\text { Pə̆-xe; } \quad \text { Je-re } \\
\text { (SA) }\end{array}$ \\
\hline red & $*_{\text {tsya }(k / \eta)}$ & ja-t6ò? & $m \partial-t \int i$ & $\begin{array}{l}\text { mə-tfə } \sim \text { mə-t } \int \mathbf{u} \sim \\
\text { a-t } \int \mathbf{u}\end{array}$ & $t \int u$ \\
\hline six & $*$ d-k-ruk & $r \dot{\varepsilon}^{\prime}$ & $\mathrm{ra}(\mathrm{m})$ & $\operatorname{re}(?)$ & 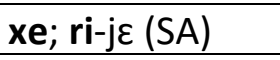 \\
\hline bite & *twak & to: & ta(?na) & $t^{\text {hai }} \sim t^{\text {ha? }}$ & $t^{h} 0-d 30$ \\
\hline frog & $*_{\mathrm{t}}(\mathrm{u} / \mathrm{i}) \mathrm{k}$ & dzo: & dzou & dzou & $\int e-d z a$ \\
\hline
\end{tabular}

Table 15. Reflexes of PTB *-k.

There are relatively few examples of the elision of the syllable-final alveolar and bilabial plosives. Elision of syllable-final ${ }^{*}-t$ appears a strong trigger for diphthongisation of the preceding vowel.

\begin{tabular}{|c|c|c|c|c|c|}
\hline Gloss & $\begin{array}{l}P T B \\
*_{-} t\end{array}$ & $\begin{array}{l}\text { Bangru } \\
* v^{\prime}(-\varnothing)\end{array}$ & $\begin{array}{l}E . M i j i \\
-\emptyset\end{array}$ & $\begin{array}{l}W . M i j i \\
-\varnothing(-?)\end{array}$ & $\begin{array}{l}\text { Hruso } \\
-\varnothing\end{array}$ \\
\hline eight & *b-r-gyat & sə-gài? & 6ə-gə & tfi-gə दagə(i) & $\int \partial-g d z \partial$ \\
\hline blow & ${ }^{*} \mathrm{k} / \mathrm{s}$-mut & màì & - & - & - \\
\hline hand, arm & ${ }^{*} \mathrm{k}(\mathrm{r}) \mathrm{u}-\mathrm{t}$ & mə-gغ̀ं? & gi & gi & Pa-gdza \\
\hline grind, crush & *kri:t & rài? & - & ri (SM) & ri (SA) \\
\hline flower & $*$ b/s-wat & mə-bua: & gə-bo & u-bo? & Jo-ba \\
\hline
\end{tabular}

Table 16. Reflexes of PTB *-t.

\begin{tabular}{llllll}
\hline Gloss & PTB & Bangru & E. Miji & W. Miji & Hruso \\
& $*-p$ & $-\varnothing\left(-v^{2}\right)$ & $-\varnothing /-m$ & $-\varnothing(-m)$ & $-\varnothing$ \\
\hline
\end{tabular}
${ }^{9}$ Palatalisation $* m y$ - $>n$ - in this lexeme is also common in the Western Tani languages (Sun
1993:126). 


\begin{tabular}{|c|c|c|c|c|c|}
\hline Gloss & $P T B$ & Bangru & E. Miji & $W . M i j i$ & Hruso \\
\hline fat (n) & ${ }^{*} s-b^{w} a(m / p)$ & mə-ba: & $\begin{array}{l}\text { məm- } \\
\text { bau/bou }\end{array}$ & məm-bau & Pว̆-bi \\
\hline cry & ${ }^{*}$ krap & k(j)e: & krəm-na & krəm-na k $\mathbf{k}^{\text {hă }}$ & $k \int 0$ \\
\hline fireplace & *g-rap & là? & - & də-thle (SM) & huk $^{\text {hi-je }(S A)}$ \\
\hline
\end{tabular}

Table 17. Reflexes of PTB *-p.

\subsection{ONSET NASALS.}

The syllable-initial $* m$ - is preserved in the Hrusish languages.

\begin{tabular}{|c|c|c|c|c|c|}
\hline Gloss & $\begin{array}{l}P T B \\
{ }^{*} m-\end{array}$ & $\begin{array}{l}\text { Bangru } \\
m-\end{array}$ & $\begin{array}{l}\text { E. Miji } \\
m-\end{array}$ & $\begin{array}{l}W . M i j i \\
m-\end{array}$ & $\begin{array}{l}\text { Hruso } \\
m-\end{array}$ \\
\hline body hair & ${ }^{*} \mathrm{~s} / \mathrm{r}-\mathrm{mul}$ & mə-mən & - & u-mu & $(\mathrm{u} / \mathrm{i}) \mathrm{mu}(\mathrm{SA})$ \\
\hline elder sister & ${ }^{*} \mathrm{ma}$ & mə-mua a-ma: & Pa-ma & a-mo a-mu & Pa-ma \\
\hline fire & *mey & $m \varepsilon:$ & mai & mai & $\mathrm{mi}$ \\
\hline vomit & $\begin{array}{l}{ }_{\mathrm{n}}^{*} \mathrm{mwa} \\
\end{array}$ & mu: & - & $\mathrm{mu}(\mathrm{SM})$ & - \\
\hline dream & $\begin{array}{l}*_{\text {r/s-mwəy; OC }} \\
*_{\text {C.məy-s }}\end{array}$ & tja-mə: & $\begin{array}{l}\text { tai-mat } \\
\text { tai-mə-na }\end{array}$ & $\begin{array}{l}\mathrm{t}^{\mathrm{h}} \mathrm{e}-\mathrm{me}(-\mathrm{ma}) \\
\sim \mathrm{t}^{\mathrm{h}} \mathrm{a}^{\mathrm{i}}-\mathrm{me}(?)\end{array}$ & $t \int^{h}$ i-mi-jo \\
\hline
\end{tabular}

Table 18. Preservation of PTB onset nasal *m-.

A change observed in Bangru and Eastern Miji is the reflex $r$ - of Proto-TibetoBurman $*_{n}$ - and, in a single attested case, syllable-initial ${ }^{*} n$-. The picture for Western Miji is ambiguous, with in some lexemes preservation of the initial $n$-, in others development into $r$ - The aberrant Bangru entry for 'year' is a probably loan from Nyasang, cf. Proto-Tani ñin (Sun 1993) versus the more regular Miji forms.

\begin{tabular}{|c|c|c|c|c|c|}
\hline Gloss & $\begin{array}{l}P T B \\
{ }^{*} n-/{ }^{*} \eta-\end{array}$ & $\begin{array}{l}\text { Bangru } \\
r-\end{array}$ & $\begin{array}{l}\text { E. Miji } \\
r-\end{array}$ & $\begin{array}{l}W . M i j i \\
n-/ r-\end{array}$ & $\begin{array}{l}\text { Hruso } \\
n-(n-)\end{array}$ \\
\hline $\begin{array}{l}\text { younger } \\
\text { sibling }\end{array}$ & *na:w & mə-rə: & $\operatorname{ran} \sim r^{\circ}$ & nu na-nu & $\begin{array}{l}\text { Po-nu; niu } \\
\text { (SA) }\end{array}$ \\
\hline two & $*(\mathrm{~g} / \mathrm{s})-\mathrm{ni}-\mathrm{s}$ & kə-rei & kə-ran k e-rə & ge-ni gə-rin & (kJə) \\
\hline near & *s-ney & mə-re: & me-roi & mə-ni & Рə-ni-sə \\
\hline listen, hear & $* r / g-n a$ & rei & roi-na & rei & - \\
\hline short & $*_{s-\eta}(i / u) \eta$ & $\begin{array}{l}\text { mə-rõn; ma } \\
\text { non }^{55} \text { (LB) }\end{array}$ & mə-run & $\begin{array}{l}\text { mə-run } \sim a \\
\text { run }\end{array}$ & - \\
\hline year & ${ }^{*}$ s-ni(:)n & anəynəท & də-ran & du-re(n) & - \\
\hline
\end{tabular}

Table 19. PTB onset nasal n-/y- to Bangru, Eastern and Western Miji r-. 
There are a number of noted exceptions to the sound change Proto-TibetoBurman * $n$ - to Bangru and Eastern Miji $r$-, as presented in Table 20. The exact conditions under which this sound change has taken place and its implications for the internal classification of the Hrusish languages remains a subject for future research, but it might be indicative of a sub-grouping of Bangru with Eastern Miji.

\begin{tabular}{|c|c|c|c|c|c|}
\hline Gloss & $\begin{array}{l}P T B \\
{ }^{*} n-/{ }^{*} \eta-\end{array}$ & $\begin{array}{l}\text { Bangru } \\
n-/ n-\end{array}$ & $\begin{array}{l}\text { E. Miji } \\
n-/ n-\end{array}$ & $\begin{array}{l}W . M i j i \\
n-/ n-\end{array}$ & $\begin{array}{l}\text { Hruso } \\
n-(n-)\end{array}$ \\
\hline ill & $*^{*}$ na- $(\mathrm{n} / \mathrm{t})$ & nua $\sim$ noa & - & no (SM) & na (SA) \\
\hline mother & *ney \̌ ni(y) & (mə/a)-ne: & ane & ani anai & a-ni \\
\hline $\begin{array}{l}\text { breast; } \\
\text { milk }\end{array}$ & ${ }^{*}$ s-nəw(k/n) & mə-nəク & mənan nag & Jufu-nu Ju-no-və & - \\
\hline $2 \mathrm{SG}$ & $*_{\text {nan }}$ & ni: $\sim$ ni: & ni & ni & - \\
\hline $1 \mathrm{SG}$ & * па-у & no: & ni-jan & $\begin{array}{l}\text { ni-jan } \\
(\mathrm{SM})\end{array}$ & no \\
\hline
\end{tabular}

Table 20. Exceptions to PTB onset nasal n-/ク- to Bangru, Eastern Miji and Western Miji r-.

\subsection{RHYMES WITH FINAL NASALS.}

Reflexes of the Proto-Tibeto-Burman syllable-final nasals are mixed. The ProtoTibeto-Burman final * $*_{-}$is commonly preserved in Bangru, as is shown in Tables 36 and 37. Proto-Tibeto-Burman syllable-final $-m$ is either elided with nasalisation of the preceding vowel or velarised in Bangru. Miji has preserved syllable-final $-m$ as syllable final alveolar or bilabial nasal in a few cases. In Hruso, the syllable-final $-m$ has been more widely attested in entries such as (?o)sam 'daughter' (SA, AA), mim 'woman' (SA, AA), ('oo)num 'younger sister' (SA, AA), arim 'tail' (SA), sam 'forest (SA), pum pom 'five' (SA, AA) and kam 'bitter' (SA).

\begin{tabular}{|c|c|c|c|c|c|}
\hline Gloss & $\begin{array}{l}P T B \\
*_{-} m\end{array}$ & $\begin{array}{l}\text { Bangru } \\
-\varnothing\end{array}$ & $\begin{array}{l}\text { E. Miji } \\
-\eta(-\varnothing /-m)\end{array}$ & $\begin{array}{l}W . M i j i \\
-n /-\eta(-\varnothing)\end{array}$ & $\begin{array}{l}\text { Hruso } \\
-\varnothing\end{array}$ \\
\hline sleep & ${ }^{*} \mathrm{~g}-(\mathrm{d}) \mathrm{z}(\mathrm{i} / \mathrm{u}) \mathrm{m}$ & dze: & dzi(na) & dzi & dxu-mudzo; dzu (SA) \\
\hline iron & *syam & s̃̃: & - & $\operatorname{sen}(S M)$ & so (SA) \\
\hline otter & *s-ram & sẽ: & - & - & si-ze (SA) \\
\hline pillow & ${ }^{*} \mathrm{~m}-\mathrm{k}(\mathrm{u} / \mathrm{i}) \mathrm{m}$ & 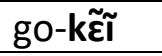 & go-kəm & (nu/wo)k $k^{h} \partial n$ & di-kJo \\
\hline egg & $*(r / l) u m$ & do:-rein & do-rin & do-ri(n) & dze-dze \\
\hline heart & $*_{m} \mathrm{~m}-\mathrm{lu}(\mathrm{n} / \mathrm{m})$ & $\begin{array}{l}\text { mə-lon- } \\
\text { wə: }\end{array}$ & $\begin{array}{l}\text { lun-və"k } \sim \text { lon- } \\
\text { və }\end{array}$ & lun-vəu $k$ & Pă-lă-biu \\
\hline
\end{tabular}

Table 21. Reflexes of PTB *-m. 


\subsection{REFLEXES OF SONORANTS *LAND*R.}

In onset position, Proto-Tibeto-Burman $* l$ - generally corresponds with a lateral approximant $l$ - in the Hrusish languages.

\begin{tabular}{|c|c|c|c|c|c|}
\hline Gloss & $\begin{array}{l}P T B \\
* I-\end{array}$ & $\begin{array}{l}\text { Bangru } \\
\text { I- }\end{array}$ & $\begin{array}{l}\text { E. Miji } \\
\text { I- }\end{array}$ & $\begin{array}{l}\text { W. Miji } \\
\text { l- (t-) }\end{array}$ & $\begin{array}{l}\text { Hruso } \\
\text { I- }\end{array}$ \\
\hline leg & *la & mə-lq & lei & lai & ə]i-lə-bu (SA) \\
\hline full & $*(p / b)$ lin & lən-bəla & $\begin{array}{l}\text { len } \sim \text { lan- } \\
\text { dan }\end{array}$ & $\begin{array}{l}\text { len } \sim \text { len } \sim p^{h_{i}}- \\
\text { tan; o-lin }(S M)\end{array}$ & - \\
\hline heart & ${ }^{*} \mathrm{~m}-\mathrm{lu}(\mathrm{n} / \mathrm{m})$ & $\begin{array}{l}\text { mə-lon- } \\
\text { wə: }\end{array}$ & $\begin{array}{l}\text { lun-vəuk } \\
\sim \text { lon-və }\end{array}$ & lun-vəuk & Pă-lă-biu \\
\hline month & ${ }^{*} \mathrm{~s}-(\mathrm{g}) \mathrm{la}$ & lù? & lo & lo lu & - \\
\hline swallow & *mlyəw-k & pə-lja pe: & - & bə-lui (SM) & - \\
\hline take & $\begin{array}{l}*(\mathrm{~g} / \mathrm{m} / \mathrm{s}) \text {-lay } \\
\not<(\mathrm{r} / \mathrm{s}) \text {-ley }\end{array}$ & lo: & lo-na & $\begin{array}{l}\text { lə } \sim \text { ho-tə } ~ \\
\text { təP-ne }\end{array}$ & la-d3o \\
\hline sell & ${ }^{*}(\mathrm{~g} / \mathrm{m} / \mathrm{s})$-lay & la: & laə-na & $1 a^{\circ}$ & - \\
\hline
\end{tabular}

Table 22. PTB *l-> Bangru l-, Miji l-, Hruso l-.

In a few instances, the reflex of Proto-Tibeto-Burman $* l$ - is a trill or fricative in Bangru and Hruso, whereas the Miji reflexes can be a retention of the lateral approximant or change into a trill.

\begin{tabular}{|c|c|c|c|c|c|}
\hline Gloss & $\begin{array}{l}P T B \\
* /-\end{array}$ & $\begin{array}{l}\text { Bangru } \\
r-\end{array}$ & $\begin{array}{l}\text { E. Miji } \\
\text { I- (r-) }\end{array}$ & $\begin{array}{l}\text { W. Miji } \\
\text { I- (r-) }\end{array}$ & $\begin{array}{l}\text { Hruso } \\
r-\end{array}$ \\
\hline road & *lam & re:-bõ & la-ban & la-bay lem-bay & $\begin{array}{l}\text { xa-bo; ra-bo } \\
\text { (SA) }\end{array}$ \\
\hline bow & $*(\mathrm{~g} / \mathrm{b} / \mathrm{m})-\mathrm{la}-\mathrm{y}$ & kə-ràì? & kə̆-rai & gə-ri g-ri $\sim \mathrm{k}^{\mathrm{h}} \mathrm{i}-\mathrm{ri}$ & $k^{h} \mathrm{i}-r i$ \\
\hline four & *b-Іәу & 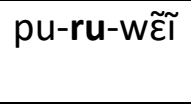 & pə-le $e^{i}$ & bə-le $e^{i}$ b-li & $\begin{array}{l}\text { psǎ-xi; } \\
\text { (SA) }\end{array}$ \\
\hline
\end{tabular}

Table 23. PTB *l-> Bangru r-, Miji l- $\sim r-$, Hruso $r-\sim x-$.

In Miji we additionally observe a change from Proto-Tibeto-Burman $* l-$ to the palatal approximant $j-$ and, in a secondary development, to fricatives $f-\sim d z-$. Lada Puroik, spoken in the Eastern Miji area, shows a similar development.

\begin{tabular}{|c|c|c|c|c|c|c|}
\hline Gloss & $\begin{array}{l}P T B \\
* /-\end{array}$ & $\begin{array}{l}\text { Bangru } \\
\text { I- }\end{array}$ & $\begin{array}{l}\text { E. Miji } \\
j-(I-)\end{array}$ & $\begin{array}{l}W . M i j i \\
j-\left(\int-/ d z^{-}\right)\end{array}$ & & $\begin{array}{l}\text { Hruso } \\
\text { I- }\end{array}$ \\
\hline heavy & ${ }^{*} \mathrm{~s}-l ә y-\mathrm{t}$ & la-təo & mə-le $e^{i}$ & mə-ji & & liu (SA) \\
\hline lift & *lan & Iõ(り) & jan i-jan-na & jan Jan & & lo (SA) \\
\hline tongue & $\begin{array}{l}{ }^{*} \mathrm{~m} / \mathrm{s} \text {-lay } \\
\text { s-ley }\end{array}$ & pə-lja: & ja-k ${ }^{h} e / k^{h} i j o$ & $\begin{array}{l}\text { dza-kihi } \\
\text { ja?-k }{ }^{\mathrm{h} i}\end{array}$ & 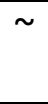 & $\begin{array}{l}\text { Pedza-bla; } \\
\text { ze-bla (SA) }\end{array}$ \\
\hline
\end{tabular}




\begin{tabular}{llllll}
\hline Gloss & PTB & Bangru & E. Miji & W. Miji & Hruso \\
\hline wind & ${ }^{*}$ g-ləy & loa & jo & jo & lau (SA) \\
\hline
\end{tabular}

Table 24. PTB *l-> Bangru l-, Miji j-, Hruso l-.

As reflexes of Proto-Tibeto-Burman ${ }^{*} r$-, we generally find trill $r$ - in Bangru and Miji and the phoneme(s) that has been variably transcribed as $\langle r, x, y, b\rangle$, in Hruso.

\begin{tabular}{|c|c|c|c|c|c|}
\hline Gloss & $\begin{array}{l}P T B \\
*_{r-}\end{array}$ & $\begin{array}{l}\text { Bangru } \\
r-\end{array}$ & $\begin{array}{l}E . M i j i \\
r-\end{array}$ & $\begin{array}{l}W . M i j i \\
r-\end{array}$ & $\begin{array}{l}\text { Hruso } \\
r-/ x-\end{array}$ \\
\hline fight & *ray & wa-re: & - & nau-ri (SM) & $t^{h}$ o-ri (SA) \\
\hline six & $* d-k-r u k$ & rè? & $\mathrm{ra}(\mathrm{m})$ & re(?) & xe; ri-jع (SA) \\
\hline grind, crush & *kri:t & raì $^{2}$ & - & $\operatorname{ri}(S M)$ & ri $(S A)$ \\
\hline far & $*_{s-r(i / u) \eta}$ & a-rəy & mə-rən & $\begin{array}{l}\text { ma-ran a-rən mə- } \\
\text { rən }\end{array}$ & Рә-хә; ә-ra (SA) \\
\hline fear & $*_{k r i}(y)$ & ni-ri-mغे? & - & $\operatorname{rin}(S M)$ & ri-je (SA) \\
\hline egg & $*(r / I) u m$ & do:-rein & do-ri(n) & do-ri(n) & - \\
\hline
\end{tabular}

Table 25. PTB *r-> Bangru r-, Miji r-, Hruso r-/x-.

In a few very basic lexemes, reflexes of Proto-Tibeto-Burman $*_{r}$ - are a retention of $r$ - in Bangru and Hruso, but change to $l$ - in Western Miji, with ambiguity in Eastern Miji.

\begin{tabular}{|c|c|c|c|c|c|}
\hline Gloss & $\begin{array}{l}P T B \\
r-\end{array}$ & $\begin{array}{l}\text { Bangru } \\
r-\end{array}$ & $\begin{array}{l}\text { E. Miji } \\
\text { I-/r- }\end{array}$ & $\begin{array}{l}\text { W. Miji } \\
\text { I- }\end{array}$ & $\begin{array}{l}\text { Hruso } \\
r-\end{array}$ \\
\hline leaf & *rwak & mə-rj’̀? & - & mə-le u-le? & Pə̆-xe; fe-re (SA) \\
\hline salt & *g-ryum & ru: & la & lu & 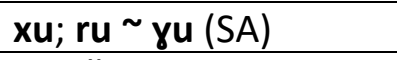 \\
\hline bone & ${ }^{*} \mathrm{~m} / \mathrm{g} / \mathrm{s}$-rus & mə-ri: & ma-rian & mi-lian & Рə̆-xə̆-be; e-rə-bje (SA) \\
\hline
\end{tabular}

Table 26. PTB $*_{r-}>$ Bangru, Miji $r-\sim l-$, Hruso $r-/ x-$.

\subsection{ORIGIN OF HRUSISH FRICATIVES AND AFFRICATES.}

Proto-Tibeto-Burman *ts- and *dz- are preserved as affricates $\overline{t \varphi}-\overline{t t}$ - and $\overline{d \bar{z}}-\overline{d z}$ in the Hrusish languages. Several examples, such as the entries for 'itch' and 'eat', show a voicing contrast between the reconstructed Proto-Tibeto-Burman forms and the Hrusish reflexes.

\begin{tabular}{|c|c|c|c|c|c|}
\hline Gloss & $\begin{array}{l}\text { PTB } \\
{ }^{*} t s-\end{array}$ & $\begin{array}{l}\text { Bangru } \\
\frac{16}{-}\end{array}$ & $\begin{array}{l}\text { E. Miji } \\
\widehat{t f-}\end{array}$ & $\begin{array}{l}W . M i j i \\
\widehat{t f-}\end{array}$ & $\begin{array}{l}\text { Hruso } \\
\overparen{t \int-}\end{array}$ \\
\hline wing & *g-(t)syəw-k/n & mə-tढ̆̀े? & - & gə-t]i (SM) & - \\
\hline
\end{tabular}




\begin{tabular}{|c|c|c|c|c|c|}
\hline Gloss & $\begin{array}{l}P T B \\
*_{t s-}\end{array}$ & $\begin{array}{l}\text { Bangru } \\
\frac{16^{-}}{}\end{array}$ & $\begin{array}{l}\text { E. Miji } \\
\widehat{t f-}\end{array}$ & $\frac{W . M i j i}{t_{f-}}$ & $\begin{array}{l}\text { Hruso } \\
\widehat{t f-}\end{array}$ \\
\hline red & $*_{\text {tsya }(k / \eta)}$ & ja-ţढ̀े? & mə-t $\mathbf{f i}$ & $\begin{array}{l}\text { mə-tfə } \sim \mathrm{m} ə- \\
\mathrm{t} \int \mathbf{u} \sim a-t \int u\end{array}$ & $t \int u$ \\
\hline finger & $*(\mathrm{t}) \mathrm{s}(\mathrm{y}) \mathrm{ow}$ & məge-t6oa & gi-tfo? & gi-tfo? & Pĕd3ə-tJə \\
\hline
\end{tabular}

Table 27. Reflexes of PTB*(t)s(y)-.

\begin{tabular}{|c|c|c|c|c|c|}
\hline Gloss & $\begin{array}{l}P T B \\
* d z-\end{array}$ & $\begin{array}{l}\text { Bangru } \\
\widetilde{d z^{-}}-\widetilde{t \epsilon-}\end{array}$ & $\begin{array}{l}\text { E. Miji } \\
d z-(t)-)\end{array}$ & $\begin{array}{l}W . M i j i \\
d z-/ t--\end{array}$ & $\begin{array}{l}\text { Hruso } \\
\text { tf- }\left(d z-/ \int-\right)\end{array}$ \\
\hline $\begin{array}{l}\text { hot, } \\
\text { warm }\end{array}$ & $*$ tsa-t $\Varangle$ dza-t & 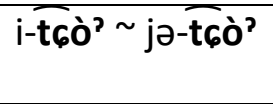 & mə-dzu & $\begin{array}{l}\text { a-dziu mə- } \\
\text { dz(i)u }\end{array}$ & Ju; Jəu (SA) \\
\hline eat & ${ }^{*} \mathrm{~N}-\mathrm{dz}(\mathrm{y}) \mathrm{a}-\mathrm{k} / \mathrm{n} / \mathrm{t} / \mathrm{s}$ & tढ̆ù? tढ̆ & $t \int u \sim t \int \partial$ & $t \int u$ & tJa \\
\hline sleep & ${ }^{*} \mathrm{~g}-(\mathrm{d}) \mathrm{z}(\mathrm{i} / \mathrm{u}) \mathrm{m}$ & $\overline{\mathrm{dz}} \mathbf{e}:$ & dzi-na & d3i & $\begin{array}{l}\text { dxu-mudzo; } \\
\text { dzu (SA) }\end{array}$ \\
\hline thorn & $*^{*} \mathrm{~m}-(\mathrm{d}) \mathrm{z}(\mathrm{y}) \mathrm{u}(:) \mathrm{k}$ & 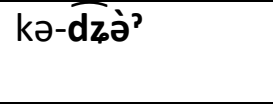 & - & $\begin{array}{l}\text { gə(dzik/dzu) } \\
\sim \text { bi-t } \int u\end{array}$ & ku-t $\int u$ \\
\hline
\end{tabular}

Table 28. Reflexes of PTB*(d)z(y).

Examples of palatalisation and affrication of Proto-Tibeto-Burman $* t$ - and $* d$ can be found in Tables 10 and 11 and include Bangru $\overline{d z} \partial$ :, Miji dzou, Hruso Je$d_{3} a$ 'frog' from Proto-Tibeto-Burman *t(u/i)k FROG and Bangru gadzun $\sim d z o \eta \sim d z o$ ? , Hruso $t \int u-d z u$ 'spear' from Proto-Tibeto-Burman *m-duy $>$ m-day SPEAR. The examples in Tables 10 and 11 also show that affrication in Miji and Hruso is in an advanced stage compared to Bangru.

\subsection{VOWEL REFLEXES.}

This section will present the main reflexes of Proto-Tibeto-Burman vowels in the Hrusish languages. These reflexes are summarised in Table 29, equally frequent realisations are after slashes and marginal realisations are between parentheses.

\begin{tabular}{|c|c|c|c|c|c|c|}
\hline$P T B$ & Bangru & Eastern Miji & Western Miji & Hruso & $\#$ & Table \# \\
\hline$*_{\text {-a }}$ & $-\mathrm{O}\left(-\grave{o}^{?}\right)$ & - & - & $-\mathrm{o}(\mathrm{u})$ & 4 & 30 \\
\hline$*_{\text {-a }}$ & $-\mathrm{u}\left(-\mathrm{u}^{\mathrm{P}}\right)$ & $-u(-\partial /-u)$ & $-u(-a /-2 /-i)$ & $-u(-a /-\partial)$ & 6 & 31 \\
\hline$*_{-a C}$ & -o/-ò?/-õ & $-u(-a C /-i)$ & $-u(-a C /-\partial /-i)$ & -o/-u (-am) & 6 & 32 \\
\hline$*_{-\mathrm{aC}}$ & $-a /-a^{2}$ & $(-\tilde{e} /-\mathrm{au})$ & $(-\partial /-e /-a u)$ & $-\mathrm{i}(-\partial)$ & 5 & 33 \\
\hline$*_{-\mathrm{aC}}$ & $-\dot{\varepsilon}^{?} /-\varepsilon$ & - & $-\mathrm{eC}$ & $-\mathrm{e}$ & 3 & 34 \\
\hline$*_{-\mathrm{aC}}$ & $-\mathrm{u}$ & -2 & $-\mathrm{u}$ & $-\mathrm{u}$ & 1 & 35 \\
\hline$*_{-} \mathrm{u}(\mathrm{C}) /-\mathrm{i}(\mathrm{C})$ & $-\partial(\eta)$ & (mixed) & (mixed) & $-\partial(-u /-a)$ & 9 & 36 \\
\hline$*_{-u(C)}$ & -u (-up/-on) & -on (-un) & -un (-on) & $-u(-\partial)$ & 2 & 37 \\
\hline${ }^{*} \mathrm{u}(\mathrm{C})$ & $-i\left(-\grave{\varepsilon}^{2}\right)$ & (mixed) & (mixed) & (mixed) & 3 & 38 \\
\hline$*_{-\mathrm{iC} /-\mathrm{uC}}$ & $-\varepsilon \mathrm{i}(\mathrm{C}) /-\mathrm{ai}$ & $-\partial(C) /-i(C)$ & $-\partial(C) /-i$ & $-\partial(-u /-0 /-i)$ & 8 & 39 \\
\hline
\end{tabular}




\begin{tabular}{|c|c|c|c|c|c|c|}
\hline *-әу & -ai (-ci/-i) & -ei & -i (-ei) & $-i(-\partial /-u)$ & 7 & 40 \\
\hline *-ay/-ey & $-\varepsilon\left(-a i^{?}\right)$ & -ai (-oi/-i/-e) & $-\mathrm{i}(-\mathrm{a} /-\mathrm{ai} /-\mathrm{e})$ & $-\mathrm{a} /-\mathrm{i}$ & 8 & 41 \\
\hline$*_{\text {-əw }(\mathrm{C})}$ & $-\partial(C)(-u /-o)$ & $-\partial(C)(-a(C))$ & $-\partial(-u /-i /-\partial u)$ & $-\partial(-a /-y)$ & 6 & 42 \\
\hline$*_{\text {-aw/-ow }}$ & $-\mathrm{o} /-\mathrm{u}$ & $(-\mathrm{ou} /-\mathrm{i})$ & $(-0 P /-ə /-u(C))$ & $(-\mathrm{uo})$ & 3 & 43 \\
\hline
\end{tabular}

Table 29. Summary of reflexes of monophthong and diphthong vowels.

Sun (1993: 378) earlier reported a change from the Proto-Tibeto-Burman vowel $*_{-} a$ to vowel $-u$ in open syllables for Miji. The data indicate that whereas this observation generally holds for Miji, the Bangru picture partially reflects the Tani reflex $-O$ (Sun 1993: 308), and Hruso shows even more diverse reflexes.

\begin{tabular}{|c|c|c|c|c|}
\hline Gloss & $\begin{array}{l}P T B \\
*_{-} a \\
\end{array}$ & $\begin{array}{l}\text { Bangru } \\
-o\left(-\grave{o}^{2}\right)\end{array}$ & $M i j i$ & $\begin{array}{l}\text { Hruso } \\
-o(u)\end{array}$ \\
\hline $1 \mathrm{SG}$ & *na-y $\Varangle * k a$ & no: & - & no \\
\hline hard & $*_{\text {s-ra }}$ & mə-lo: & - & go-you go-rou (SA) \\
\hline nose & *ka そ*na & mi-ko: məлә-ko: & - & - \\
\hline penis & *la & mə-lò? & - & - \\
\hline
\end{tabular}

Table 30. PTB *-a to Bangru -o.

\begin{tabular}{|c|c|c|c|c|c|}
\hline Gloss & $\begin{array}{l}P T B \\
*_{-} a\end{array}$ & $\begin{array}{l}\text { Bangru } \\
-u\left(-\grave{u}^{2}\right)\end{array}$ & $\begin{array}{l}\text { E. Miji } \\
-u(-\partial /-i)\end{array}$ & $\begin{array}{l}W . M i j i \\
-u(-a /-\partial /-i)\end{array}$ & $\begin{array}{l}\text { Hruso } \\
(-u /-a /-\partial /-o)\end{array}$ \\
\hline month & ${ }^{*} \mathrm{~s}-(\mathrm{g}) \mathrm{la}$ & lù? & la & lo lu & - \\
\hline neg. imp. & $*(t / d) a$ & tu- & - & $\mathbf{t}^{\text {h }} \mathbf{a}-(S M)$ & $\bar{d} \mathbf{3} u-(S A)$ \\
\hline vomit & ${ }^{*}$ mwa $\Varangle$ mya-n & mu: & - & mu (SM) & - \\
\hline five & *l/b-na & рә-пu: & pu-nu & $\begin{array}{l}\text { bu-nu bo- } \\
\text { gu pu-gu }\end{array}$ & pom pum (SA) \\
\hline eat & ${ }^{*} \mathrm{~N}-\mathrm{dz}(\mathrm{y}) \mathrm{a}-\mathrm{k} / \mathrm{n} / \mathrm{t} / \mathrm{s}$ & tढ़ù? & $\mathrm{t} \int \mathrm{u} \sim \mathrm{t} \int \partial$ & $t \int u$ & $t \int a$ \\
\hline meat & *sya-n & sù? Gù? & si-kijun & si-țun & tsə sə (SA) \\
\hline
\end{tabular}

Table 31. PTB *-a to Bangru $-u$.

In closed syllables the reflexes of the Proto-Tibeto-Burman vowel $*_{-} a$ are mixed and apparently independent of phonotactic conditions, resulting in $-o,-a,-\varepsilon$ and even $-u$. Similar variation is also observed in Miji and Hruso.

\begin{tabular}{|c|c|c|c|c|c|}
\hline Gloss & $\begin{array}{l}P T B \\
*_{-} a C \\
\end{array}$ & $\begin{array}{l}\text { Bangru } \\
-o /-o^{\prime} /-\tilde{o}\end{array}$ & $\begin{array}{l}\text { E. Miji } \\
-u(-a C /-i)\end{array}$ & $\begin{array}{l}W . M i j i \\
-u(-a C /-\partial /-i)\end{array}$ & $\begin{array}{l}\text { Hruso } \\
-o /-u(-a C)\end{array}$ \\
\hline bite & $*_{\text {twak }}$ & to: & ta(?na) & $t^{\text {hai }} \sim t^{\text {ha? }}$ & $t^{h} 0-d z 0$ \\
\hline bitter & $*^{*}$ b-ka-(n/m/n) & kə-kjo: & mə-k $\mathbf{k}^{\mathbf{h}} \mathbf{u}$ & a/mə-k ${ }^{\mathbf{h}} \mathbf{u}$ & kam-jo \\
\hline $\begin{array}{l}\text { hot, } \\
\text { warm }\end{array}$ & $*$ tsa-t $\Varangle$ dza-t & 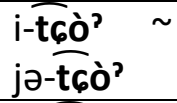 & mə-dzu & a-dziu mə-dz(i)u & $\int u$ \\
\hline red & $*_{\text {tsya }(k / \eta)}$ & ja-tढ़ò? & mə-t]i & $\mathrm{m} \boldsymbol{-}-\mathrm{t} \int \boldsymbol{\partial} \sim \mathrm{m} \boldsymbol{-}-\mathrm{t} \int \mathbf{u} \sim \mathrm{a}-\mathrm{t} \int \mathbf{u}$ & $t \int u$ \\
\hline look; see & *kan & gõ: & gan-na & wan & ho-dzo \\
\hline
\end{tabular}




\begin{tabular}{llllll}
\hline Gloss & $P T B$ & Bangru & E. Miji & W. Miji & Hruso \\
\hline lift & ${ }^{*}$ lan & lõ(n) & jan $\sim$ i-jan-na & jan jan & lo (SA) \\
\hline
\end{tabular}

Table 32. PTB *-aC to Bangru - .

\begin{tabular}{|c|c|c|c|c|c|}
\hline Gloss & $\begin{array}{l}P T B \\
*_{a C}\end{array}$ & $\begin{array}{l}\text { Bangru } \\
-a /-\grave{a}^{?}\end{array}$ & $\begin{array}{l}\text { E. Miji } \\
(-\tilde{e} /-a u)\end{array}$ & $\begin{array}{l}W . M i j i \\
(-e C /-a u /-\partial)\end{array}$ & $\begin{array}{l}\text { Hruso } \\
-i(-\partial)\end{array}$ \\
\hline eye & ${ }^{*}$ s-myak & me-jà? & mẽ & mə-ri $\sim$ me? & ?i-ni \\
\hline fat (n) & ${ }^{*} s-b^{w} a(m / p)$ & mə-ba: & məm-bau & məm-bau & Pว̆-bi \\
\hline fireplace & *g-rap & là? & - & də-thle (SM) & - \\
\hline flow & $\begin{array}{l}{ }^{*} \text { sywar } \\
\text { sywa-n/t }\end{array}$ & sa: & - & - & - \\
\hline louse & ${ }^{*} s(y) \operatorname{ar}$ & sà? & - & - & Sə (SA) \\
\hline
\end{tabular}

Table 33. PTB *-aC to Bangru $-a$.

\begin{tabular}{|c|c|c|c|c|c|}
\hline Gloss & $\begin{array}{l}P T B \\
*_{a C}\end{array}$ & $\begin{array}{l}\text { Bangru } \\
-\dot{\varepsilon}^{\top} /-\varepsilon\end{array}$ & $\begin{array}{l}\text { E. Miji } \\
-\end{array}$ & $\begin{array}{l}W . M i j i \\
-e(C)\end{array}$ & $\begin{array}{l}\text { Hruso } \\
-e\end{array}$ \\
\hline split & *pryak & pre: & - & - & - \\
\hline leech & ${ }^{*} k-r-p^{w} a t$ & 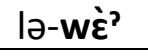 & - & də-ve (SM) & - \\
\hline leaf & *rwak & mə-rj’̀ & - & mə-le u-le? & Pə̆-xe; Je-re (SA) \\
\hline
\end{tabular}

Table 34. PTB *-aC to Bangru $-\varepsilon$.

\begin{tabular}{llllll}
\hline Gloss & PTB & Bangru & E. Miji & W. Miji & Hruso \\
& $-a C$ & $-u$ & $-\partial$ & $-u$ & $-u$ \\
\hline tooth & $*$ twan & mə-tu: & tə & $\mathbf{t}^{\text {h }} \mathbf{u} \sim$ tu & ?e-th $\mathbf{u} \sim$ ə-t $\mathbf{j u}(\mathrm{SA})$ \\
\hline
\end{tabular}

Table 35. PTB *-aC to Bangru -u.

In general, PTB $*_{-} u(C)$ and $*_{-i} i(C)$ are reduced in Bangru to $-\partial(\eta)$. The reflexes for Miji and Hruso are far less consistent.

\begin{tabular}{|c|c|c|c|c|c|}
\hline Gloss & $\begin{array}{l}P T B \\
*_{-} u(C) /- \\
i(C)\end{array}$ & $\begin{array}{l}\text { Bangru } \\
-\partial(\eta)\end{array}$ & $\begin{array}{l}\text { E. Miji } \\
\text { (mixed) }\end{array}$ & $\begin{array}{l}W . M i j i \\
\text { (mixed) }\end{array}$ & $\begin{array}{l}\text { Hruso } \\
-ə(-u /-a)\end{array}$ \\
\hline spear & $\begin{array}{l}{ }^{*} \mathrm{~m} \text {-dun } \Varangle \\
\mathrm{m} \text {-dan }\end{array}$ & gə-tढ̆ & dzon d30? & dzun & $t \int u-d z u$ \\
\hline $\begin{array}{l}\text { body hair, } \\
\text { feather }\end{array}$ & $* s / r-m u l$ & mə-mən & - & u-mu & - \\
\hline frog & $*_{\mathrm{t}}(\mathrm{u} / \mathrm{i}) \mathrm{k}$ & d3o: & dzou & dzou & Sedza \\
\hline vagina & $*_{\text {tsyuk }}$ & 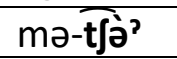 & - & - & - \\
\hline hair (head) & ${ }^{*}$ s-pu & gә-рә: & gopal gopə & $\begin{array}{l}\text { wo- } \mathbf{p}^{\text {hijo }} \sim \text { u- } \\
\mathbf{p}^{\mathbf{h}^{\mathbf{u}}}\end{array}$ & - \\
\hline live, grow up & ${ }^{*}$ s-rin & san & - & Jan (SM) & 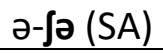 \\
\hline
\end{tabular}




\begin{tabular}{|c|c|c|c|c|c|}
\hline Gloss & $\begin{array}{l}P T B \\
{ }^{*}-u(C) /- \\
i(C)\end{array}$ & $\begin{array}{l}\text { Bangru } \\
-\partial(\eta)\end{array}$ & $\begin{array}{l}\text { E. Miji } \\
\text { (mixed) }\end{array}$ & $\begin{array}{l}W . M i j i \\
\text { (mixed) }\end{array}$ & $\begin{array}{l}\text { Hruso } \\
- \text {-a }(-u /-a)\end{array}$ \\
\hline far & ${ }^{*} s-r(i / u) \eta$ & a-rəy & mə-rən & $\begin{array}{l}\text { ma-ran a- } \\
\text { rən mə-rən }\end{array}$ & Рә-хә; ә-ra (SA) \\
\hline full & $*(p / b)$ lin & Iən-bəla & len $\sim$ lon-dan & 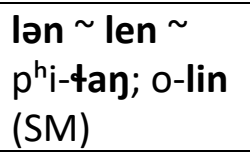 & - \\
\hline name & $*_{r-m i(\eta / n)}$ & mə-mjən & mãrn & mãrn & $\begin{array}{l}\text { Pənә-nә; Pani-ni } \\
\text { (SA) }\end{array}$ \\
\hline
\end{tabular}

Table 36. Reflexes of PTB rhyme ${ }_{-}-u(C)$ and ${ }^{*}-i(C)$.

There are a number of noted exceptions to this rule, in which PTB *-u(C) corresponds to Bangru $-u,-o \eta$ or $-u \eta$.

\begin{tabular}{|c|c|c|c|c|c|}
\hline Gloss & $\begin{array}{l}P T B \\
*_{-u(C)}\end{array}$ & $\begin{array}{l}\text { Bangru } \\
-u(-u \eta /-o \eta)\end{array}$ & 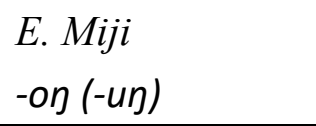 & $\begin{array}{l}W . M i j i \\
- \text { un (-op) }\end{array}$ & $\begin{array}{l}\text { Hruso } \\
-u(-\partial)\end{array}$ \\
\hline drink & $* N / s-t u n$ & ton $\sim$ tun & tonna $\sim t^{\text {h }}$ on & $\mathbf{t}^{\mathrm{h}}$ on $\sim \mathrm{t}^{\mathrm{h}} \mathrm{un}(\mathrm{SM})$ & $t^{h} u-d z o$ \\
\hline heart & ${ }^{*} \mathrm{~m}-\mathrm{lu}(\mathrm{n} / \mathrm{m})$ & mə-lon-wə: & lun-və"uk lon-və & lun-vəuk & Pă-lă-biu \\
\hline child & $* \mathrm{~m}-(\mathbf{t} / \mathbf{d}) \mathbf{u}$ & mə-ब्dzu: & - & - & - \\
\hline guts & $*_{r}-\mathbf{g y u}-\eta$ & mə-lə-gu: & - & - & - \\
\hline white & $*$ plu & lәрu: & - & - & - \\
\hline
\end{tabular}

Table 37. PTB *-u(C) to Bangru -u (-up/-op).

Additional exceptions include the ones in Table 38.

\begin{tabular}{|c|c|c|c|c|c|}
\hline Gloss & $\begin{array}{l}P T B \\
*_{u(C)}\end{array}$ & $\begin{array}{l}\text { Bangru } \\
-i\left(-\dot{\varepsilon}^{2}\right)\end{array}$ & $\begin{array}{l}\text { E. Miji } \\
\text { (mixed) }\end{array}$ & $\begin{array}{l}W . \text { Miji } \\
\text { (mixed) }\end{array}$ & $\begin{array}{l}\text { Hruso } \\
\text { (mixed) }\end{array}$ \\
\hline bone & $*_{\mathrm{s}} / \mathrm{m} / \mathrm{g}$-rus & mə-ri: & ma-rian & mi-lian & Pă-xə̆-be; e-rə-bje (SA) \\
\hline who & *su & tiiwo & təə & $t \int^{h} u$ & $\overline{d 3 u}$ \\
\hline six & *d-k-ruk & $r \grave{\varepsilon}^{2}$ & $\mathrm{ra}(\mathrm{m})$ & re(?) & $x e$; rij $\varepsilon(S A)$ \\
\hline
\end{tabular}

Table 38. Exceptions to the reflexes of PTB rhyme *-u(C).

Furthermore, as Table 39 shows, Proto-Tibeto-Burman vowels $*_{-i} i$ and $*_{-} u$ followed by a syllable final alveolar fricative, stop or nasal or bilabial nasal correspond with diphthongs in modern Bangru, a uniquely Bangru development not shared by Miji or Hruso. Proto-Tibeto-Burman nasal coda *-n/- $\eta$ commonly results in nasalisation of the diphthong, and elision of final alveolar plosive $-t$ resulted in falling pitch and glottal stop. One exception is the reflex of the ProtoTibeto-Burman root SLEEP $*_{g-}(d) z(i / u) m$, Bangru $\widehat{d} z e:$ Miji $\widehat{d_{3}} i$, Hruso $d u u \sim$ $\widehat{d} u$ shown in Table 21, perhaps as a result of allophamic variation between root 
$\# 127 *_{s-y}(i / u) p$ and $\# 128 * g-(d) z(i / u) m$. The Bangru and Miji reflexes of ProtoTibeto-Burman *bi- $\eta$ GOAT, Bangru sə-pẽ̃ , Eastern Miji fa-pen 6u-prem, Western Miji tfe-prem $\sim \int \partial-p h \breve{r r \partial n}$, strongly suggest a Proto-Tibeto-Burman root with syllable-final biliabial nasal *bi-m.

\begin{tabular}{|c|c|c|c|c|c|}
\hline Gloss & $\begin{array}{l}P T B \\
*_{-i C /-u C}\end{array}$ & $\begin{array}{l}\text { Bangru } \\
-\varepsilon i(C) /-a i\end{array}$ & $\begin{array}{l}E . M i j i \\
-\partial(C) /-i(C)\end{array}$ & $\begin{array}{l}W . M i j i \\
-\partial(C) /-i\end{array}$ & $\begin{array}{l}\text { Hruso } \\
-ə(-u /-o /-i)\end{array}$ \\
\hline egg & $*(r / I)$ um & do:-rein & do-rin & do-ri(n) & - \\
\hline pillow & ${ }^{*} \mathrm{~m}-\mathrm{k}(\mathrm{u} / \mathrm{i}) \mathrm{m}$ & 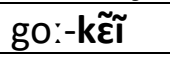 & go-kəm & (nu/wo)khon & di-kJo \\
\hline three & *g-sum & 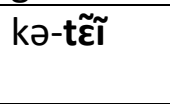 & $k^{\mathrm{h}} \partial$-təm & $\begin{array}{l}\text { gə-tən } \sim \text { kə-th }{ }^{\mathrm{h}} \\
\sim \mathrm{k}^{\mathrm{h}} \text { ə-təm }\end{array}$ & d3ə \\
\hline liver & ${ }^{*} \mathrm{~m}-\sin$ & 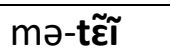 & - & mə-thən (SM) & ě-djǔ (SA) \\
\hline blow & ${ }^{*} \mathrm{k} / \mathrm{s}-\mathrm{mut}$ & mài? & - & - & - \\
\hline grind, crush & $*_{\text {kri:t }}$ & ràì? & - & ri $(\mathrm{SM})$ & ri $(S A)$ \\
\hline hand, arm & ${ }^{*} \mathrm{k}(\mathrm{r}) \mathrm{u}-\mathrm{t}$ & mə-gغ̀ं? & gi & gi & ?a-gdzə \\
\hline two & $*(g / s)-n i-s$ & kə-rei & $k^{h}$ e-ro & gə-rin ge-ni & $k \int \partial$ \\
\hline
\end{tabular}

Table 39. Diphthongisation of PTB vowels $*_{i}$ and $*_{u}$ in Bangru.

Bangru shares the Tani development of Proto-Tibeto-Burman diphthong *-əy to monophthong vowel $-i$ in two lexemes, but like Eastern Miji has most commonly preserved a diphthong vowel. Western Miji and Hruso have generally monophthongised to vowel $-i$.

\begin{tabular}{|c|c|c|c|c|c|}
\hline Gloss & $\begin{array}{l}P T B \\
\text {-әу }\end{array}$ & $\begin{array}{l}\text { Bangru } \\
\text {-ai (-عi/-i) }\end{array}$ & $\begin{array}{l}\text { E. Miji } \\
-e i\end{array}$ & $\begin{array}{l}W . M i j i \\
-i(-e i)\end{array}$ & $\begin{array}{l}\text { Hruso } \\
-i(-\partial /-u)\end{array}$ \\
\hline think & *məy & mi: & - & mjen (SM) & - \\
\hline rain & ${ }^{*} \mathrm{~m}-\mathrm{t}(\mathrm{w})$ әу $(\mathrm{n})$ & nə-di: & - & - & 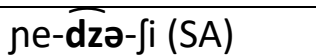 \\
\hline give & *s-bəy-n/k & bai & ba $^{i} \sim$ be $^{i}-n a$ & $\begin{array}{l}\mathbf{p}^{\mathrm{h}} \text { e-bi } \sim \mathbf{b i} \sim \\
\text { bip-ne }\end{array}$ & d3i-d30 \\
\hline four & *b-lәу & pu-ruwẽ̃ & pə-le $e^{i}$ & bə-le $e^{i} \sim$ bli & psă-xi p $^{h_{i}} \mathbf{i}-$ i $(S A)$ \\
\hline rub & *sywəy & рә-sci & - & - & - \\
\hline excrement & *kləy & lai & - & - & - \\
\hline die & *səy & tai & tei-na & $t \int i$ & dzu; dzi (SA) \\
\hline
\end{tabular}

Table 40. Reflexes of PTB diphthong *-əy.

In general, the reflex of Proto-Tibeto-Burman diphthongs *-ay and *-ey is Bangru

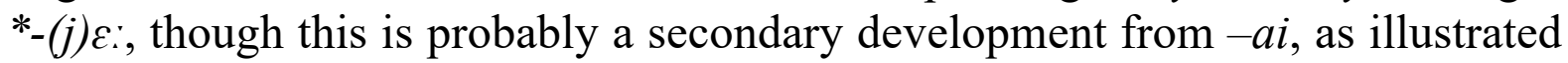
by the noted exception 'bow'. The common Hruso and Western Miji reflexes of $*_{-a y}$ is $-a$, and of $*_{-} e y$ is $-i$, whilst Eastern Miji tends to reflect the diphthongs.

\begin{tabular}{llllll}
\hline Gloss & PTB & Bangru & E. Miji & W. Miji & Hruso \\
& $*-a y /-e y$ & $-\varepsilon\left(-a i^{2}\right)$ & $-a i(-o i /-i /-e)$ & $-i(-a /-a i /-e)$ & $-a /-i$ \\
\hline
\end{tabular}




\begin{tabular}{|c|c|c|c|c|c|}
\hline Gloss & $P T B$ & Bangru & E. Miji & $W . M i j i$ & Hruso \\
\hline make; do & *day & dja $\sim \mathbf{d} \varepsilon:$ & - & - & da-d3o \\
\hline bow & *(g/b/m)-la-y & kə-rài & kə̆-rai & ge-ri $\sim k^{h} i-r i$ & $\mathrm{k}^{\mathrm{h}} \mathrm{i}-\mathrm{ri}$ \\
\hline fight & *ray & wa-re: & - & nau-rij (SM) & $\mathrm{t}^{\mathrm{h}} \mathrm{o}-\mathrm{ri}(\mathrm{SA})$ \\
\hline tongue & $\begin{array}{l}{ }^{*} \mathrm{~m} / \mathrm{s} \text {-lay } \gtrsim \mathrm{s}- \\
\text { ley }\end{array}$ & pə-lje: & ja(-k $\left.{ }^{h} e / k^{h} i j o^{i}\right)$ & dza-kihi $\sim$ ja?-k ${ }^{h_{i}}$ & Pedza-bla \\
\hline fire & *mey & $m \varepsilon:$ & mai & mai & $\mathrm{mi}$ \\
\hline seed & *sey & mə-te $\tilde{\varepsilon}^{\prime}$ & tai-loi & $\mathbf{t}^{\mathrm{h}} \mathbf{e}-\mathrm{li} \sim \mathbf{t}^{\mathrm{h}} \mathbf{e}-\mathrm{d} 30$ & - \\
\hline near & *s-ney & mə-re: & mə-ro ${ }^{i}$ & mə-ni a-nin & Рə-ni-sə \\
\hline mother & ${ }^{*}$ ney $\Varangle$ ni(y) & (mə/a)ne: & a-ne & a-nai a-ni & a-ni \\
\hline
\end{tabular}

Table 41. Reflexes of PTB diphthongs *-ay and $*_{-}$ey.

Proto-Tibeto-Burman diphthong *-əw usually corresponds to Bangru -ə, but marginally vowels $-u$ and $-O$ also occur, a development mirrored in Hruso and Miji.

\begin{tabular}{|c|c|c|c|c|c|}
\hline Gloss & $P T B$ & Bangru & E. Miji & $W . M i j i$ & Hruso \\
\hline & $-\partial w$ & $-\partial(C)(-u /-0)$ & $-\partial(C)(-a(C))$ & $-\partial(-u /-i /-\partial u)$ & $-\partial(-a /-y)$ \\
\hline $\begin{array}{l}\text { breast; } \\
\text { milk }\end{array}$ & *s-nəw(k/n) & mə-nəク & mənay nag & $\begin{array}{l}\text { Jufu-nu } \sim \text { Ju- } \\
\text { no-və }\end{array}$ & - \\
\hline steal & $*_{r-k \partial w}$ & lə-kə: & 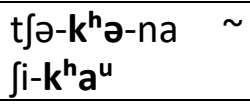 & 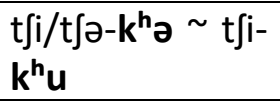 & 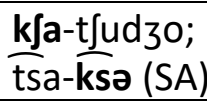 \\
\hline smoke & ${ }^{*} k \partial w-n / t$ & me-kə: & mai-k ${ }^{\text {h}} \partial n$ & mai-k ${ }^{\text {h}} \partial n$ & mŭ-kJə \\
\hline snake & *bəw & bə: & ba $^{u}$ & (na-)bəu $\sim \mathbf{b}^{\mathbf{h}} \mathbf{u}$ & by \\
\hline wing & $*^{*} \mathrm{~g}$-(t)syəw-k/p & mə-tढ़ù? & - & gə-tfi (SM) & - \\
\hline $\begin{array}{l}\text { elder } \\
\text { brother }\end{array}$ & $*(g / k) \partial w-n$ & a-ko: & kə-voi & $\begin{array}{l}\text { a-khə-vo } \sim \text { a- } \\
\text { ke-vo } \sim k^{\text {h}} \partial- \\
\text { vo? }\end{array}$ & - \\
\hline
\end{tabular}

Table 42. Reflexes of PTB diphthong *-əw.

Proto-Tibeto-Burman diphthong *-aw (*-ow) generally corresponds to Bangru simple vowels $-u /-o$.

\begin{tabular}{|c|c|c|c|c|c|}
\hline Gloss & $\begin{array}{l}P T B \\
*_{-a w / *^{*} o w}\end{array}$ & $\begin{array}{l}\text { Bangru } \\
-o(-u)\end{array}$ & $\begin{array}{l}\text { E. Miji } \\
\text { (-ou/-i) }\end{array}$ & $\begin{array}{l}\text { W. Miji } \\
(-o(\text { P)/-ə/-uC) }\end{array}$ & $\begin{array}{l}\text { Hruso } \\
(- \text {-uo) }\end{array}$ \\
\hline lick & ${ }^{*}$ s-lya:w & kə-lo: & - & - & - \\
\hline bird & *daw OR *dow & 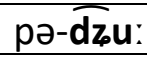 & bə-dzi & bə-dzə bu-dzu? & - \\
\hline chicken & *daw OR *dow & do: & dou & dok do? dup & d3uo \\
\hline
\end{tabular}

Table 43. Simplification of PTB diphthongs $*_{-}$aw and $*_{-o w}$.

\section{LEXICAL ISOGLOSSES}


On basis of our current understanding of the phonology of Bangru and the sound changes from Proto-Tibeto-Burman to Bangru, we were able to identify satisfactory etymologies in almost $50 \%$ of the lexical entries. For the remaining $50 \%$, in quite a number of cases we found cognates of Bangru with other regional reconstructed proto-languages, presented in section 5.1. In section 5.2, we present some isoglosses with reconstructed proto-languages that also exhibit the Proto-

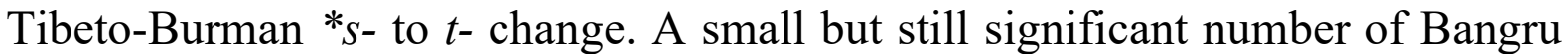
forms appears to have Hrusish cognates but no, or only scattered, cognates in other languages, presented in section 5.3. For want of space, most of the supporting data can be found in the appendices B and C. Wherever possible, we have made a first preliminary attempt at reconstructing Proto-Hrusish forms, based on our Bangru data and the comparative Miji and Hruso data and the observations in the previous section.

\subsection{ISOGLOSSES WITH NEIGHBOURING LANGUAGES}

The influence of the Western Tani language Nyasang is clearly reflected in Bangru. The table in appendix B presents a summary of some of the most obvious Nyasang loans in Bangru. Despite these loans, a large proportion of the Bangru vocabulary remains distinctly non-Tani. Illustrative of this is perhaps the fact that there are very few correspondences between the fifty 'characteristic Tani roots' presented by Sun (1993: 430) and Bangru, and borrowing of these cannot be excluded. In fact, for the vast majority of these fifty items, Bangru shows perfectly regular reflexes of Proto-Tibeto-Burman roots. Puroik appears to be another important contact and/or substrate language for Bangru, with around 50 shared lexical isoglosses between the two languages. Most of these are reflexes of a shared Proto-Tibeto-Burman root. Table 44 presents some Proto-Hrusish reconstructions ${ }^{10}$ together with possible isoglosses in languages spoken in the immediate vicinity of the Hrusish languages. The complete set of underlying data can be found in Appendix C.

\begin{tabular}{|c|c|c|c|}
\hline Entry & Bangru/Miji/Hruso & $\mathrm{PH}$ & Other proto-forms, isoglosses \\
\hline sun & $\overline{\mathrm{d} z ̧}_{\mathrm{u}} \mathrm{p} / \mathrm{d} 30 \mathrm{p} / \mathrm{d} z \mathrm{u}$ & $* \overline{\mathrm{d} z u}$ ? & Bokar dun-ñi, Bengni do:-ñi (PT) \\
\hline $\begin{array}{l}\text { paddy } \\
\text { rice }\end{array}$ & 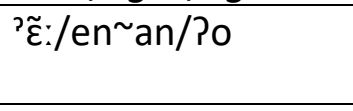 & *an & $\begin{array}{l}\text { PT *am-buin RICE (UNCOOKED); } \\
\text { Rawang } \operatorname{am}^{33} \text { (LP) }\end{array}$ \\
\hline woman & nəwe:/nəmrai/- & *nə-mə-raj & - \\
\hline soil & nò?/na?/no & *na? & $\begin{array}{l}\text { PT *mron; Bengni ño; BG nak; SS k }{ }^{h} n \tilde{~} \\
\sim \text { k }^{h} \text { nũ: }\end{array}$ \\
\hline
\end{tabular}

\footnotetext{
${ }^{10}$ These reconstructions reflect the most likely form at the Proto-Hrusish level, based on the sound correspondences between Proto-Tibeto-Burman and Bangru and internal sound correspondences. Indeterminate consonants are indicated by $\mathrm{C}$, indeterminate nasal by $\mathrm{N}$, indeterminate vowels by $\mathrm{V}$.
} 


\begin{tabular}{|c|c|c|c|}
\hline Entry & Bangru/Miji/Hruso & $P H$ & Other proto-forms, isoglosses \\
\hline long & $\begin{array}{l}\text { məpan/məpijan/pJ } \\
\text { u }\end{array}$ & *mə-pjan & 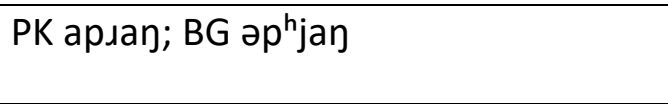 \\
\hline deep & $\begin{array}{l}\text { mərju:/arək mərə } \\
\sim \text { mərup } \sim \text { marik/rju }\end{array}$ & *mə-ruk & $\begin{array}{l}\text { PT *run; PKC *ruak EMPTY; SS 'aruk } \\
\text { 'empty' }\end{array}$ \\
\hline ten & rəワ/lən/xə & *rən & PT *rjun; PTk *ra \\
\hline hundred & lə刀/bəlun/phuyu & *bə-lun & PT *Iun; also Koro pələ (AK) \\
\hline do & ru:/ru/- & *ru & $\begin{array}{l}\text { PT rju; PNN *rə:y; BG rjet; SS ra(t/P) } \\
\text { re(t/P); Nyasang ri; Bengni rji }\end{array}$ \\
\hline cut & $\begin{array}{l}\mathrm{t} \varepsilon / \mathrm{t}^{\mathrm{h}} \mathrm{a}^{\mathrm{i}}(\mathrm{ne})^{\sim} \mathrm{ta} \mathrm{i}^{-} \\
\mathrm{na} / \mathrm{d} z \varepsilon \sim \mathrm{d} 30-\mathrm{jo}\end{array}$ & $*$ taj & $\begin{array}{l}\text { PTk *tat; PT *tək; TS tok; Kayan thài } \\
\text { 'cut surface, plough' (MK) }\end{array}$ \\
\hline friend & 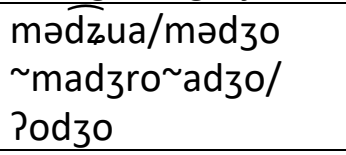 & 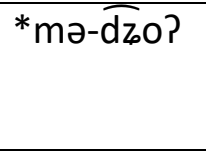 & PT *ј̌(o/u)n * *̌en; PR a-dua \\
\hline house & $\begin{array}{l}\text { ne:/nan nam } \sim \text { ne } \\
\text { neon/se }\end{array}$ & *nam & $\begin{array}{l}\text { PTB \#5746 *nam VILLAGE; Galo namə } \\
\text { (GLDC } 09 \text { Galo); Koro ne (AK); Baram } \\
\text { nam (Kansakar 2010) 'house' }\end{array}$ \\
\hline take & lə:/lə 4ə/la & *la & $\begin{array}{l}\text { PT *lan; PKC *laa-I, laak-II; TS la; KD la; } \\
\text { PK lei rei. }\end{array}$ \\
\hline $\begin{array}{l}\text { excrete, } \\
\text { defecate }\end{array}$ & (lai) $\widehat{t} 6 a i /-\widehat{t s i}$ & *tsəaj & 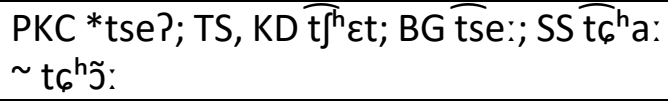 \\
\hline waterfall & kuase(n)/-/ ksətsu & *kua-su? & PK kuasua? \\
\hline ant & $\begin{array}{l}\text { səni:/Sinin } \sim \text { cəui/sin } \\
\text { i }\end{array}$ & $*$ si-ni & Bugun Ginin \\
\hline road & 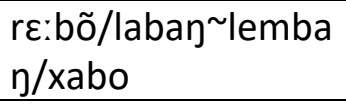 & *lam-ban & Apatani lem-bo (PT); TM lemdan \\
\hline $\begin{array}{l}\text { younger } \\
\text { sibling }\end{array}$ & $\begin{array}{l}\text { mərə:/rə nu〜nanu } \\
\text { /Ponu }\end{array}$ & *mə-nun & SS mirin 'younger sister of man' \\
\hline salt & ru:/lə /u/ru & $* \operatorname{lu}$ & PT lo, Nyishi a-lu \\
\hline this & $\begin{array}{l}\text { həy/hunlo hunji h } \\
\text { ãnai/heji ho }\end{array}$ & *hun & PK həђ; Galo ‘hi (GLDC) \\
\hline
\end{tabular}

Table 44. Lexical isoglosses with neighbouring languages.

\subsection{ISOGLOSSES WITH OTHER NORTHEAST INDIAN LANGUAGES}

Some of the lexemes for which we did not find reconstructed Proto-TibetoBurman roots have cognates in the Kuki-Chin, Tangkhulic and Central Naga languages. These isoglosses deserve extra attention because they have reconstructed proto-forms and these languages share the Proto-Tibeto-Burman $*_{s}$ - to $t$ - change, and might be further evidence of a larger genetic sub-grouping of Tibeto-Burman languages in Northeast India.

\begin{tabular}{llll}
\hline Entry & Bangru/Miji/Hruso & PH & Proto-forms, cognates \\
\hline knife & we:tsən/va'tJonzi/vetsə & *vaj-tsun & PKC *tsem \\
\hline
\end{tabular}




\begin{tabular}{|c|c|c|c|}
\hline Entry & Bangru/Miji/Hruso & $P H$ & Proto-forms, cognates \\
\hline cloud & $\begin{array}{l}\text { wa:wə:/mimu } \sim \text { m'emy } \sim \\
\text { maimay } \sim \text { maim mər/məmə }\end{array}$ & $\begin{array}{l}\text { *maj- }^{\text {mawn }} \\
\text { məwn }\end{array}$ & $\begin{array}{l}\text { PKC *may; PT *m(ə/u)k; PTk } \\
{ }^{*} \text { moj }\end{array}$ \\
\hline where & kə:/khəjo $k^{h} i j a \sim k^{h} \partial k^{\sim} k^{h} r o i / h a g \partial$ & ${ }^{*} \mathrm{k} \partial ?$ & PKC *koy \2 khoy \ hoy \\
\hline spicy & 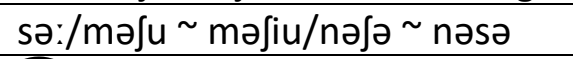 & ${ }^{*} \mathrm{~m}$-su & PTk*sa \\
\hline sweet & 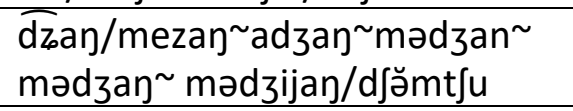 & ${ }^{*}$ mə-jan & PCN *a-m-jan; BG p ${ }^{\text {hijan }}$ \\
\hline snow & 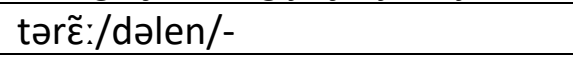 & *də-ren & PCN *ra(?) ICE / SNOW \\
\hline boil (water) & lu:/-/lusu $\times u \int \partial \int ə$ & $* \operatorname{lu}$ & $\mathrm{PCN} * \mathrm{~m}$-lu \\
\hline
\end{tabular}

Table 45. Lexical isoglosses with Northeast Indian languages.

\subsection{HRUSISH LEXICAL ISOGLOSSES.}

Finally, there are a number of lexical forms shared between Bangru and at least one of the other Hrusish languages for which we could find only a few isolated cognates or no cognates at all elsewhere. Lack of data from many other TibetoBurman languages is probably a major cause, but perhaps some lexemes represent unique Hrusish lexical isoglosses. Full data are again available in appendix C.

\begin{tabular}{|c|c|c|c|}
\hline Entry & Bangru/Miji/Hruso & $\mathrm{PH}$ & Cognates \\
\hline with (together) & dżərə/dzoru/-dza & $* \overline{\mathrm{d} z} \mathrm{~V} r u$ & \\
\hline awake & $\mathrm{pi}: / p^{h} r \partial u / p^{h_{i}}$ & *pri & \\
\hline bee & 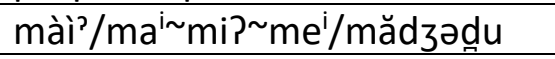 & $*_{\text {majk }}$ & \\
\hline garlic, onion & 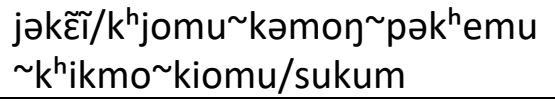 & *kik-mun & \\
\hline straight & məkəro:/mugəray/gədou & *mə-gə-ran & PTB \#2160 (p/b)lyan? \\
\hline new & $\begin{array}{l}\text { məkərə:/məkərə〜məgəniu } \\
\text { agənu/Păkə̆n }\end{array}$ & *mə-gə-nu & \\
\hline seven & 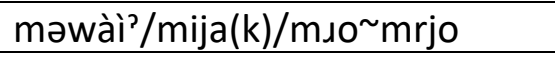 & *mə-ljak & BG məlija: \\
\hline nine & sətəク/sətən/sta & *sə-tin & \\
\hline cloth & gì?/grə/gije & *gi-le & Koro gile (AK) \\
\hline ring (finger-) & gəle?/gile gilan/gzətsəalje & *gə-leC & Koro lale (AK) \\
\hline light & 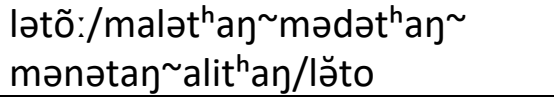 & *mə-lə-tan & \\
\hline spittle & jè?/3e/- & *je? & \\
\hline (swidden) field & wa:/vaw/- & *wa & \\
\hline door & nepãi/banpi banp $^{\text {hik }} v^{\text {vaphi/- }}$ & *pin & BG ha:pin \\
\hline broom & $\begin{array}{l}\text { sja:/na } \int i \sim \text { nesi namjoi } \\
\text { namøoirə/neso }\end{array}$ & *(nam)sjaj & \\
\hline yesterday & təga:/degau təga/- & *də-gaC & \\
\hline day & 'agu:/hũe/- & *ga & \\
\hline what & $\operatorname{ta\eta } / \tan \sim \tan \sim t^{\text {hen }} \sim \operatorname{tin} /-$ & *tin & $\mathrm{SS} * \mathrm{ti}$ \\
\hline how many & kəләru:/kəло/khəла & *kə-na & \\
\hline
\end{tabular}




\begin{tabular}{|c|c|c|c|}
\hline Entry & Bangru/Miji/Hruso & $P H$ & Cognates \\
\hline dry & 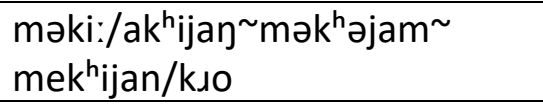 & *mə-ki-lan & \\
\hline short & mərõn/mərun arun/udu & *mo-nun & \\
\hline old & 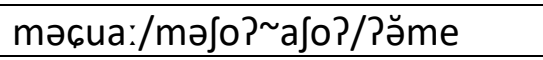 & ${ }^{*} \mathrm{~m} 2-60 ?$ & \\
\hline thigh & məru:/lug/- & *mə-lu & TS lum \\
\hline sky & $\begin{array}{l}\text { nədəlu:/gijan (na)dzang/ } \\
\text { medzə } \sim \text { nədzə }\end{array}$ & *nə-də-lan & \\
\hline vegetable & $p e: / p^{h} e n / p^{h} i j \varepsilon$ & *pen & \\
\hline night & 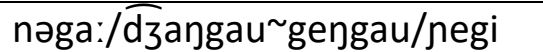 & *nə-gaC & \\
\hline $2 \mathrm{PL}$ & dze: /dzil//dzo & $* \overline{\mathrm{d}} \mathrm{z} \mathrm{V}$ & \\
\hline cook & $k u: / k^{h} u \sim k 2^{\sim} k^{h} \partial n e / k^{h} u$ & *ku & \\
\hline aconite & nәрว̃/nәр ${ }^{h} a \eta /-$ & *nə-pan & \\
\hline horn & 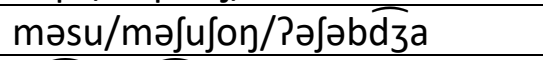 & ${ }^{*}$ mə-su & \\
\hline that & petढ़i/paitjəlo/- & ${ }^{*}$ paj-tढ़i & \\
\hline know & 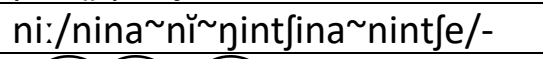 & $*$ ni & \\
\hline dirty & nət $\int \mathrm{i} / \mathrm{nit} \int \mathrm{i} / \mathrm{\text {nits }}$ & ${ }^{*}$ ni-tढिi & \\
\hline liquor & 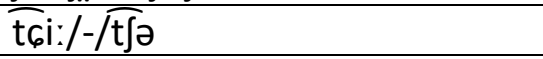 & *t6i & \\
\hline mortar & ləra:/dəlo/rulu & $*$ Iu-IV & \\
\hline pestle & pəla:/bəlau/- & *bə-laC & \\
\hline fish & tsədzəme:/tri t $^{\mathrm{h}} \mathrm{ri} \sim \mathrm{t} \int \mathrm{o}^{\mathrm{i}} / \mathrm{t} \int^{\mathrm{h}} \mathrm{e}$ & $*_{\operatorname{trV}}$ & \\
\hline wood & go:/u go/Jo & $*(\mathrm{~g}) \mathrm{o}$ & \\
\hline laugh & tua/tho tona/th ${ }^{\mathrm{h}}$ əjo & $*_{\text {toC }}$ & \\
\hline speak & te:/ta/t $\int^{\mathrm{h}} \mathrm{ed} 30$ & *təwC & $\begin{array}{l}\text { OC taw-s; Kayan tài } \\
(\mathrm{MK}) ; \text { Burmese tãi } \\
(\mathrm{TBL})\end{array}$ \\
\hline root & 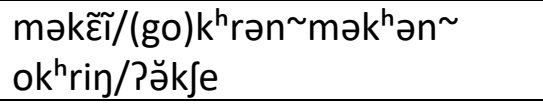 & ${ }^{*}$ mə-krin & \\
\hline stand & gi:/gijon gəron/gudzu & *gu & \\
\hline walk & dja:/dai/dzu & $*$ daj & TS dI; Dulong di ${ }^{53}$ (TBL) \\
\hline pig & jù?/dzo(?) dzio dzuo/- & *jow? & \\
\hline water & wi:/və/- & *wi & $\begin{array}{l}\text { Guiqiong } \text { wi }^{53} \quad(\mathrm{ZM}) \text {; } \\
\text { Jiulong Pumi wi }\end{array}$ \\
\hline tell & 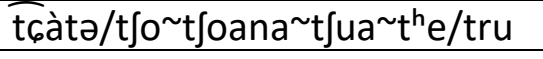 & $* \overline{t 6 a C}$ & \\
\hline bad & 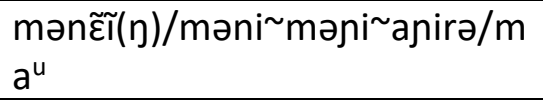 & *mə-nin & \\
\hline few & mi:gəmع/mija me?/me? & *mej? & \\
\hline
\end{tabular}

Table 46. Possible Hrusish lexical isoglosses.

\section{CONCLUSIONS}

In Table 5 we presented a summary of the main sound changes that we have hitherto identified for the Proto-Tibeto-Burman consonants. Similarly, Table 29 presents the main reflexes of Proto-Tibeto-Burman monophthong and diphthong vowels. Although none of these individual sound changes can be considered 
unique to the Hrusish cluster within the Tibeto-Burman language family, the combination of these individual sound changes is a strong indication for a shared phonological history of these individual linguistic varieties.

The Proto-Tibeto-Burman $*_{S-}$ to Hrusish $t$ - change is not unique in the Tibeto-Burman language family and has been attested for the Kuki-Chin, BodoGaro, Central Naga and Tangkhulic languages as well as Karbi. But none of the surrounding languages such as the Tani languages, the Kho-Bwa languages and the Bodic languages have participated in this sound change. In the surrounding Tani and Kho-Bwa languages, syllable-final plosives are generally preserved, whereas the Hrusish languages exhibit a strong tendency towards open syllables. Similarly, the Tani and Bodo-Garo languages and the Kho-Bwa languages Puroik, Khispi and Duhumbi preserve syllable-final nasal consonants, whereas the Hrusish languages exhibit a tendency for weakening or loss of syllable-final nasals, with as noted exception the velar nasal. The phonological development from Proto-Tibeto-Burman ${ }^{*} n$ - to $r$ - observed in a number of Hrusish lexemes is only shared to some extent by Eastern Puroik. Miji and Lada Puroik share the phonological change from Proto-Tibeto-Burman $l$ - to $j$ - in a small number of lexemes. This development has not been observed in Bangru or Hruso and neither in the Tani languages or other Puroik varieties. The consistent loss of ProtoTibeto-Burman syllable-final ${ }^{*}-l$ and ${ }^{*}-r$ in the Hrusish languages differentiates these languages from the neigbouring Tani languages, which have preserved these consonants in coda position.

Miji has rather consistent reflex $-u$ of Proto-Tibeto-Burman ${ }^{*}-a$ in open syllables, whereas Bangru shows alternation with the outcome $-O$, also observed in the Tani languages. The Kho-Bwa languages generally preserve the ProtoTibeto-Burman syllable-final *a. Unlike Miji and Hruso, Bangru has diphthong reflexes of the Proto-Tibeto-Burman syllable-final consonants ${ }^{*}-i C$ and $-u C$.

Our data thus present additional evidence for Sun's 1993 assumption that Bangru's closest genetic relatives are the languages of Shafer's 'Hruso', i.e. Miji and Hruso. Possible substrate languages, lexical and phonological innovations and borrowing from contact languages have long masked the affiliation of this cluster. The phonological aberrance of particularly Hruso was already noticed by Shafer (1947: 184) who commented that

\section{“...dialect $A$ [Miji] (...) is considerably more archaic than dialect $B$ [Hruso]..."}

The present paper also indicates that a considerable number of lexemes in the Hrusish languages do not fit well with hitherto reconstructed Proto-TibetoBurman roots. A fair number of these lexemes reconstructs well at the ProtoHrusish level and there are also a number of regional cognates for these reconstructed roots. This might contribute to the reconstruction of additional roots 
for Proto-Tibeto-Burman and will be an interesting, if not challenging subject for future research.

Although most Bangru speakers are convinced of the continued viability of their language, the survival of Bangru is threatened by the rapid spread of Nyasang as the locally and regionally dominant language. In mixed Bangru-Nyasang households, Nyasang often becomes the mother tongue of the children, who might not have more than a passive command of Bangru. Outside the household, Hindi and Nyishi are the languages of communication, and the role of Nyishi is strengthened by the rapid spread of various Christian denominations in the area, for whom Nyishi is the liturgical language. Hopefully, this description will encourage other linguists to write a comprehensive descriptive grammar of the language, for which we would gladly make our data and analyses available.

\section{REFERENCES}

Anderson, J.D. 1896. A short vocabulary of the Aka language. Shillong: Assam Secretariat Printing Office.

Anderson, Gregory D.S. 2014. On the classification of the Hruso (Aka) language. Paper presented at the $20^{\text {th }}$ Himalayan Languages Symposium, Singapore, July 162014.

Abraham, P.T. 2005. A grammar of Nyishi language. Delhi: Farsight Publishers. Abraham, Binny, Kara Sako, Elina Kinny \& Isapdaile Zeliang. 2005. A sociolinguistic research among selected groups in Western Arunachal Pradesh highlighting Monpa. Unpublished report.

Baxter, William and Laurent Sagart. 2014. Old Chinese: A new reconstruction. Oxford: Oxford University Press.

Benedict, Paul K. 1972. Sino-Tibetan: A Conspectus. Cambridge: Cambridge University Press.

Blench, Roger and Mark W. Post. 2014. Rethinking Sino-Tibetan phylogeny from the perspective of North East Indian languages. In Thomas Owen-Smith and Nathan W. Hill (eds.). Trans-Himalayan Linguistics, 71-104. Berlin: Mouton deGruyter.

Blench, Roger. 2014. The origins of nominal affixes in MSEA languages: convergence, contact and some African parallels. In N. J. Enfield and Bernard Comrie (eds.). Languages of Mainland Southeast Asia: The state of the Art, 550-577. Berlin: Mouton de Gruyter. Accessed June 152014 from https://rogerblench.academia.edu/RogerBlench.

Bruhn, Daniel W. 2014. A phonological reconstruction of Proto-Central Naga. Ph.D. Dissertation, University of California, Berkeley. Accessed via STEDT database June 162014 from http://stedt.berkeley.edu/search/. 
Driem, George van. 2011. The Trans-Himalayan phylum and its implications for population prehistory. Communication on Contemporary Anthropology, 5, 135-142.

Driem, George van. 2014. Trans-Himalayan. In Thomas Owen-Smith and Nathan W. Hill (eds.). Trans-Himalayan Linguistics, 11-40. Berlin: Mouton deGruyter.

French, Walter T. 1983. Northern Naga: A Tibeto-Burman Mesolanguage. PhD dissertation, New York: The City University of New York.

Galo Welfare Society. 2009. Galo-English dictionary. Itanagar: Galo Welfare Sociery.

Haspelmath, Martin and Uri Tadmor (eds.), 2009. Loanwords in the World's Languages: A Comparative Handbook. Berlin and New York: Mouton de Gruyter.

Joseph U.V. and Robbins Burling. 2006. The comparative phonology of the Boro Garo languages. Mysore: Central Institute of Indian Languages.

Kansakar, Tej Ratna. 2010. Baram wordlist. Unpublished manuscript. Accessed via STEDT database http://stedt. berkeley.edu/search on 2014-09-06.

Klaproth, Julius Heinrich von. 1823. Asia Polyglotta. Paris: A. Schubart.

LaPolla Randy J. 2003. Overview of Sino-Tibetan morphosyntax. In Graham Thurgood and Randy J. LaPolla (eds.) The Sino-Tibetan languages, 22-42. London: Routledge.

Lǐ, Dàqín 李大勤. 2003. 崩如语概况 Bēngrú-yǔ gaìkuàng [Bangru language overview]. 民族语文 Mínzú Yǔwén [Minority Languages], no. 5: 64-80.

Manson, Ken. 2010. Kayan-English/English-Kayan dictionary. Unpublished manuscript. Accessed via STEDT database http://stedt.berkeley.edu/search/ on 2014-09-06.

Matisoff, James A. 1978. Variational Semantics in Tibeto-Burman: The "Organic" Approach in Linguistic Comparison. Occasional Papers of the Wolfenden Society on Tibeto-Burman Linguistics, Volume VI. Philadelphia: Institute for the Study of Human Issues (ISHI).

Matisoff, James A. 2003. Handbook of Proto-Tibeto-Burman: System and Philosophy of Sino-Tibetan Reconstruction. Berkeley: University of California.

Mortensen, David R. 2012. Database of Tangkhulic languages. Unpublished manuscript. Accessed via STEDT database http://stedt.berkeley.edu/search/ on 2014-09-06.

Schubert, J. 1964. Hrusso-Vokabular. Deutsche Akademie der Wissenschaften zu Berlin. Mitteilungen des Instituts für Orientforschung 10: 295-350.

Shafer, Robert. 1947. Hruso. Bulletin of the School of Oriental and African Studies 12.1: 184-196.

Shafer, Robert 1955. Classification of the Sino-Tibetan languages. Word 11(1), 94-111. 
Simon, I.M. 1979. Miji language guide. Shillong: Directorate of Research, Government of Arunachal Pradesh.

Simon, I.M. 1993 [1970]. Aka language guide. Shillong: Directorate of Research, Government of Arunachal Pradesh.

Sun, Tianshin Jackson. 1993. A historical-comparative study of the Tani (Mirish) branch in Tibeto-Burman. PhD dissertation, Berkeley: Department of Linguistics, University of California.

Sun, Hongkai et al. (1991). Zàngmiănyǔ yǔyīn hé cíhuì [Tibeto-Burman phonology and lexicon]. Beijing: Chinese Social Sciences Press.

Tame, Ramya. 2011. An ethnographic study of the Bangrus of Kurung Kumey District of Arunachal Pradesh. Unpublished M.Phil. dissertation. Doimukh: Arunachal Institute of Tribal Studies, University of Arunachal Pradesh.

Tame, Ramya. 2012. Bangrus of Arunachal Pradesh: an ethnographic profile. International Journal of Social Science Tomorrow 1 (3): 1-12.

Tame, Ramya. 2013. Tribal culture and folklore of Arunachal Pradesh: A study in Kurung Kumey district. International Journal of Research in Sociology and Social Anthropology 1 (2): 26-30.

VanBik, Kenneth. 2009. Proto-Kuki-Chin: A reconstructed ancestor of the KukiChin languages. (STEDT monograph series \#8). Berkeley: University of California.

\section{APPENDICES}

\section{APPENDIX A: LIST OF GLOSSES AND ABBREVIATIONS TO SECONDARY SOURCES.}

From own data:

BG: Bugun

KD: Khispi \& Duhumbi

NY: Nyasang/Nyishi

PK: Puroik

SS: Sartang \& Sherdukpen

TM: Tawang Monket

TS: Tshangla

WT: Written Tibetan

From secondary data:

AA: Abraham et al. (2005) Hruso

Chinese

Aka

ABN: Abraham (2005) Nyishi

AE: Abraham et al. (2005) 'Eastern'

Miji

AK: Abraham et al. (2005) Koro Aka

AS: Shafer (1947) Aka

AW: Abraham et al. (2005)

'Western' Miji

OC: Baxter Sagart (2014) Old

GL: GLDC (2009) Galo

LB: Li (2003) Bangru

LP: LaPolla 2003

MK: Manson (2010) Kayan

PBG: Joseph and Burling (2006)

Proto-Bodo-Garo

PCN: Bruhn (2014) Proto-Central-

Naga

PKC: VanBik (2009) Proto-Kuki- 
Chin

PNN: French (1983) Proto-Northern

Naga

PT: Sun (1993) Proto-Tani

PTB: Proto Tibeto-Burman STEDT

PTk: Mortensen 2012 Proto-

Tangkhulic
SA: Simon (1993 [1970]) Hruso Aka

SH: Shafer (1947) Hruso Aka

SM: Simon (1979) 'Western' Miji

TBL: Sun (1991) Tibeto-Burman lexicon

ZM: Sun (1991) Tibeto-Burman

Guide to transcription of Simon (1979): th $=\left[\theta \sim \mathrm{t}^{\mathrm{h}}\right], \mathrm{dh}=\left[\partial^{\sim} \mathrm{d}^{\mathrm{h}}\right], \mathrm{ng}=[\mathrm{\eta}], \tilde{\mathrm{n}}$

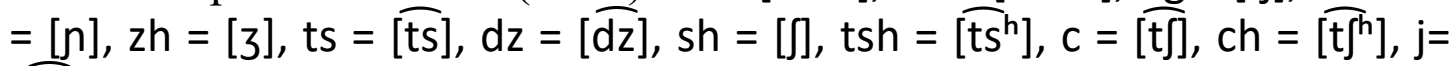
$[d 3], h l=[t], \| l=[l],-h=?, a w=[a u], i w=[i u], y=[j]$.

Guide to transcription of Simon (1993 [1970]): $\mathrm{hh}=[\mathrm{x}], \mathrm{gh}=[\mathrm{\gamma}], \mathrm{ny}=[\mathrm{n}]$,

$\mathrm{ng}=[\mathrm{\eta}]$ or vng $\left.=\tilde{\mathrm{v}}, \mathrm{i}=[\partial], \mathrm{sh}=[\mathrm{J}], \mathrm{zh}=[3], \mathrm{u}=[\mathrm{y}], \mathrm{j}=[\widehat{\mathrm{d} z}], \mathrm{c}=\widehat{[\mathrm{t}]}], \mathrm{ch}=\widehat{[\mathrm{tt}]^{\mathrm{h}}}\right]$, $\mathrm{ts}=[\widehat{\mathrm{ts}}], \mathrm{dz}=[\overline{\mathrm{dz}}], \mathrm{ks}=[\mathrm{ks}], \mathrm{gz}=[\overline{\mathrm{gz}}], \mathrm{e}=[\mathrm{e}]$ or $[\varepsilon], \mathrm{o}=[\mathrm{o}]$ or $[0]$. 
APPENDIX B: PLAUSIBLE NYASANG/TANI LOANWORDS IN BANGRU.

Unless mentioned otherwise (SM/SA), Hruso data from AA; E. Miji data from AE, Western Miji data from AW.

\begin{tabular}{|c|c|c|c|c|c|}
\hline Gloss & PTB, PT, Nyasang & Bangru & E. Miji & W. Miji & Hruso \\
\hline buffalo & mintiu (ABN); Pur. mindək & $\operatorname{mind} d(\mathrm{k})$ & 6əkəram cək $^{\text {h am }}$ & $\int u k^{h} r e \sim \int \partial k^{h} e n$ & фәтә; fumu (SA) \\
\hline sugarcane & bapih (NY) & bapi: & kəlaydzan & gəlandzan gedan & dǔgo \\
\hline mosquito & $\operatorname{tarun}(\mathrm{NY})$ & tarun & $\begin{array}{l}\text { sirdonmai } \\
\text { sirijondanmai } \\
\end{array}$ & $\begin{array}{l}\begin{array}{l}\text { sərədan } \sim \text { sirijando } \\
\text { surindan }\end{array} \\
\end{array}$ & səxən (SA) \\
\hline duck & pəḑзap (NY); PT * jap DUCK; IA hañs & pəন্dzap & ũso & ũso muso husõ & Posa; asa (SA) \\
\hline maize & tupu $(A B N)$ & tәрu: & 6ə̌ban Jibat & sibe? & sǎbe; tsibje (SA) \\
\hline chili & jamdək (NY); Pur. jandək & jəda: & dzaijo & dzaju $\sim$ dza $^{u}$ & adə \\
\hline spider & aparam (NY) & aparanga & nərija(nam) & $\begin{array}{l}\text { bafidərga }{ }^{u} \sim \text { batfibegau } ~ \\
\text { basidərgian }\end{array}$ & Jagəxa \\
\hline banana & PT *ko-pak; Pur. kopak & kכpàd(?) & Iəlay & rilang & xolo \\
\hline lizard & 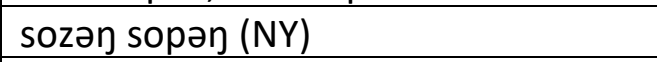 & 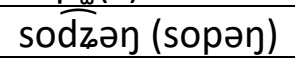 & gusuman & 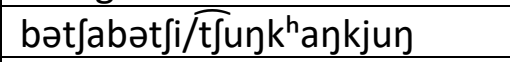 & - \\
\hline tired & api & api: & də $\sim 1 \partial$ & də la & la \\
\hline gold & in; PT *un & ain & se? san & Sə $\sim \operatorname{sen}$ & Ja \\
\hline rainbow & ha'rigo'go (ABN) & harigogo & jomonəkəi & $\begin{array}{l}\text { rumudək }^{h} r e \sim \text { dzimodək }^{h} e^{\sim} \\
\text { dzumodək }{ }^{h} r e\end{array}$ & nedziləgo; jeḑzăfə (SA) \\
\hline elbow & IaPbə力 (NY) & laabən & 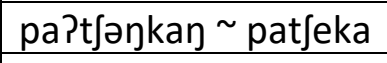 & pantrəyk ${ }^{\mathrm{h}} \mathrm{u} \sim$ patfinku & apobe \\
\hline back & kojə⿱ (NY); Pur. kət]jek & $\begin{array}{l}\text { kəjon; } \\
\text { məkəţàì }\end{array}$ & kəban & (mə)gəban & ?ə̌tfəbo; otsəbo (SA) \\
\hline knee & ləbəク (NY); PT *lə-buи & ləbəク & lekəpiu & lekəpiu $\sim$ legəp ${ }^{h} \mathrm{u} \sim$ leik ${ }^{\mathrm{h}} \mathrm{u}$ & 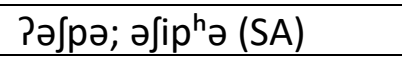 \\
\hline left & la?t]i (NY); PT *lak-ke LEFT SIDE & la?tfi & sivijo & sivijo savijo & фovo; $\mathrm{p}^{\mathrm{h}}$ əvo (SA) \\
\hline right & labje? (NY); PT *lak-bruk RIGHT SIDE & lapbjek & 6ədzen & sidzən $\sim \operatorname{sid} 3$ in & 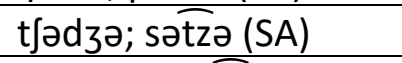 \\
\hline $\operatorname{dog}$ & PTani *pjan WILD DOG & sope: & zaza $^{i}$ & Jadzi $\sim$ sadzik Jaze? & Jyjo; səljo tsəlo (SA) \\
\hline branch & \#1278 s-ka(:)k; \#6653 PTani *hak & $a: ?$ & $\begin{array}{l}\text { gonotfan } \\
\text { mənətJan }\end{array}$ & mət $\int i t \int a^{u} \sim(o / u) d ə t \int a(\eta)$ & Joфo, ?oфotsa \\
\hline
\end{tabular}




\begin{tabular}{|c|c|c|c|c|c|}
\hline Gloss & PTB, PT, Nyasang & Bangru & E. Miji & W. Miji & Hruso \\
\hline love & $\begin{array}{l}\text { \#1359 *m-ba(k/n) LOVE/ COPULATE; PK a-pak; } \\
\text { Bengni pak (PT) }\end{array}$ & po: & məd3ovəna loran & $\begin{array}{l}\text { lundzan avirə matjodori; } \\
\text { lunzan (SM) }\end{array}$ & nalbo; nələvo (SA) \\
\hline
\end{tabular}

\section{APPENDIX C: ALPHABETICAL BANGRU LEXICON.}

Unless mentioned otherwise (SM/SA), Hruso data from AA; E. Miji data from AE, Western Miji data from AW.

\begin{tabular}{|c|c|c|c|c|c|c|c|}
\hline Gloss & PTB & Bangru & E. Miji & W. Miji & Hruso & $\mathrm{PH}$ & $\begin{array}{l}\text { Proto-forms, } \\
\text { isoglosses }\end{array}$ \\
\hline aconite & - & nәро̃: & - & nəp ${ }^{h}$ an (SM) & - & *nə-pan & - \\
\hline ant & - & səni: & 6əni & Sinin $\sim \operatorname{sini}$ & $\operatorname{sini}$ & ${ }^{*}$ si-ni & BG Jinin \\
\hline awake & - & pi: & - & $p^{h}$ rəu (SM) & $p^{h_{i}}(S A)$ & *pri & - \\
\hline axe & $\# 2772 * r-p{ }^{w a}$ & $\begin{array}{l}\text { pərdžzin } \\
\text { purudzain }\end{array}$ & pə̆lə phĕlu & bulu blu & $p^{h}$ odze (/SA) & *bə-ru( (dzin) & SS p plu; KD p pa \\
\hline bad & - & 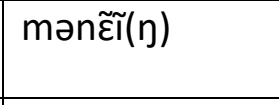 & məni & $\begin{array}{l}\text { məni məni } \\
\text { anirə }\end{array}$ & $\mathrm{ma}^{\mathrm{u}}$ & $*_{\text {mə-nin }}$ & - \\
\hline bamboo (big) & $\# 2549 * g / r-p^{w} a$ & ba: & bra $\sim$ brə & $\begin{array}{l}\text { bra }{ }^{\mathrm{u}} \sim \text { bro } ~ \\
\mathrm{~d}^{\mathrm{i}} \mathrm{u}\end{array}$ & sə & *bra & $\begin{array}{l}\text { PK məbıa: 'small } \\
\text { bamboo'; WT sba } \\
\text { 'cane, bamboo' }\end{array}$ \\
\hline bark (tree) & $\# 729 * p(r / y) a$ SKIN & gәрја: & - & oup $^{\mathrm{h}}$ ri (SM) & $\int k^{h} u(S A)$ & *(g)o-prja & - \\
\hline bear & $\# 2777$ *d-wam & sət6õ: & Jitfan & $\begin{array}{l}\text { Jitjan Si-stan; } \\
\text { Jutsang }\end{array}$ & $\begin{array}{l}\text { Jət/o; sətso } \\
\text { (SA) }\end{array}$ & *so-tsan & $\begin{array}{l}\text { PK sətəm; BG səth um; } \\
\text { SS sət }{ }^{\text {h}} u \eta ; K D \text { Gat }{ }^{\text {h}} J m\end{array}$ \\
\hline bee & - & maì? & $\mathrm{mi} ? \sim \mathrm{ma}^{\mathrm{i}}$ & $\mathrm{mi} ? \sim \mathrm{me}^{\mathrm{i}}$ & mădzədu & ${ }^{*}$ majk & - \\
\hline $\begin{array}{l}\text { big (thick, } \\
\text { wide) }\end{array}$ & $\begin{array}{l}\# 183^{*}(\mathrm{t} / \mathrm{d}) \text { ow- } n \\
\text { SWELL / THICK } \\
\end{array}$ & $\begin{array}{l}\text { mədua; məbja: } \\
\sim \text { məbe: }\end{array}$ & \begin{tabular}{|l} 
mədəbu \\
mədo
\end{tabular} & $\begin{array}{l}\text { mədo ado } \\
\text { məhin }\end{array}$ & djo; deu (SA) & *mə-do? & SS * ?adつk; KD ?วdว? \\
\hline bird & $\# 1604$ *daw OR *dow & 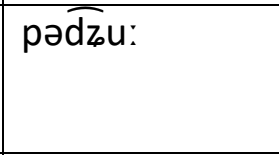 & bədzi рә6ә & $\begin{array}{l}\text { bədzə } \\
\text { budzu? bəzi'; } \\
\text { buzu (SM) }\end{array}$ & musə & *bə-dow & $\begin{array}{l}\text { PK pədou; SS bət } \bar{c}^{h} a: \\
\sim \overline{p t}^{h} \varepsilon\end{array}$ \\
\hline bite & $\begin{array}{l}\text { \#1799 *twak PECK } \\
\text { AT/BITE }\end{array}$ & to: & ta ta?na & $t^{\text {h }} a i \sim t^{\text {h }} a ?$ & $t^{\mathrm{h}}$ od30 & $*$ ta? & $\begin{array}{l}\text { WT 'thog 'pick, } \\
\text { pluck'; TS tok 'bite' }\end{array}$ \\
\hline
\end{tabular}




\begin{tabular}{|c|c|c|c|c|c|c|c|}
\hline Gloss & PTB & Bangru & E. Miji & W. Miji & Hruso & $\mathrm{PH}$ & $\begin{array}{l}\text { Proto-forms, } \\
\text { isoglosses }\end{array}$ \\
\hline bitter & $\begin{array}{l}\text { \#229 *b-ka-(n/m/n) } \\
\text { BITTER / BILE / LIVER }\end{array}$ & kəkjo: & $m \partial k^{h} u$ & $a k^{h} u \sim m \partial k^{h} u$ & kamfo & *kam & $\begin{array}{l}\text { PT *ko ka:; BG } \\
\text { ək } k^{h} \text { :; TS kha-lu }\end{array}$ \\
\hline blanket & - & kambol & kombolo & kombolo(u) & kombolo & - & IA kanbal \\
\hline blood & $\# 230$ *s-hywəy-t & ja: & medza $a^{i} z i$ & $3^{i} \sim d 3 a^{i}$ & Јə; ifə (SA) & $*_{\mathrm{jaC}}$ & TS ji $\sim$ zi \\
\hline blow & \#503 *k/s-mut & màì & - & - & - & - & - \\
\hline body & $\# 14 * d u$ & məब̄द̧ò? & $3 a^{i}$ & za? & Poso; əso (SA) & - & BG seo: \\
\hline $\begin{array}{l}\text { body hair, } \\
\text { feather }\end{array}$ & $\begin{array}{l}\# 363{ }^{*} \mathrm{~s} / \mathrm{r}-\mathrm{mul} \nvdash^{*} \mathrm{~s}- \\
\mathrm{mil} \aleph^{*} \mathrm{~s}-\mathrm{myal}\end{array}$ & məməク & - & umu & (u/i)mu (SA) & ${ }^{*}$ mə-muC & $\begin{array}{l}\text { PK amuin; SS Pamin; } \\
\text { KD mur }\end{array}$ \\
\hline boil (water) & - & lu: & dzom zəmna & $\begin{array}{l}\text { dzən dzrən; } \\
\text { ziu (SM) }\end{array}$ & $\begin{array}{l}\text { xufəfe; lusu } \\
\text { (SA) }\end{array}$ & $*$ lu & $\begin{array}{l}\text { PCN *m-lu; Idu ma }{ }^{55} \\
\text { lu }{ }^{55} \text { (ZM) }\end{array}$ \\
\hline bone & $\begin{array}{l}\# 232{ }^{*} \mathrm{~m} / \mathrm{g} / \mathrm{s}-\mathrm{rus} ; \\
\# 238{ }^{*} \mathrm{~g}-\mathrm{r}(\mathrm{w} / \mathrm{y}) \mathrm{a}(\mathrm{\eta} / \mathrm{k}) ?\end{array}$ & məri: & marian & milian & $\begin{array}{l}\text { Păxə̆be; erəbje } \\
\text { (SA) }\end{array}$ & *mə-ri-jan & - \\
\hline bow & $\begin{array}{l}\# 2386 *(\mathrm{~g} / \mathrm{b} / \mathrm{m})-\mathrm{la}-\mathrm{y} \\
\text { BOW/ ARROW }\end{array}$ & kərài? & kə̆rai & $k^{h_{i r i}} \sim g(ə) r i$ & $k^{h}$ iri & *gə-raj & BG rək; KD, SS, TS li \\
\hline breath & $\# 32$ *N-sak & sò? & - & $\operatorname{dit}^{h} u(S M)$ & - & - & $\begin{array}{l}\text { OC* *sək; Western } \\
\text { Tani *sak; PCN *sak; } \\
\text { KD 6O? 'rest'; TS Gon } \\
\text { 'rest' }\end{array}$ \\
\hline breast; milk & 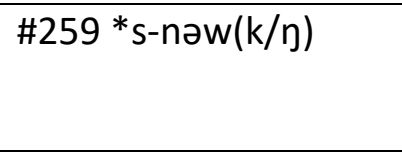 & mənəク & mənap nag & $\begin{array}{l}\text { Jufunu } \\
\text { Junovə; nu } \\
\text { (SM) }\end{array}$ & $a p^{h} u$ & *mə-nun & $\begin{array}{l}\text { SS nun nyn; TS nu; } \\
\text { KD nu }\end{array}$ \\
\hline broom & - & sja: & $\begin{array}{l}\text { namfoi } \\
\text { nam6oirə }\end{array}$ & nafi $~$ nesi & neso & *(nam)sjaj & - \\
\hline burn & $\begin{array}{l}\text { \#340 *duk BURN / } \\
\text { KINDLE }\end{array}$ & $\begin{array}{l}\text { (me:) } \text { dza: } \\
\text { blr53 (LB) }\end{array}$ & frəna & $\begin{array}{l}\text { fra }(n / \eta) \sim \\
\text { pene?; } p^{h} \text { rjan } \\
p^{\text {hen }}(S M)\end{array}$ & pejo; $p^{h} w a(S A)$ & - & - \\
\hline cane; rope & $\begin{array}{l}\text { \#533 *s-rwi(y) } \\
\text { CANE/CORD }\end{array}$ & su: 6u: & - & Ju (SM) & Ju (SA) & *su & $\begin{array}{l}\text { PKC * ruy } \Varangle \text { hruy } \\
\text { CORD/RATTAN; PCN } \\
\text { *a-(h)rəj; PT *son; }\end{array}$ \\
\hline
\end{tabular}




\begin{tabular}{|c|c|c|c|c|c|c|c|}
\hline Gloss & PTB & Bangru & E. Miji & W. Miji & Hruso & $\mathrm{PH}$ & $\begin{array}{l}\text { Proto-forms, } \\
\text { isoglosses }\end{array}$ \\
\hline & & & & & & & $\begin{array}{l}\text { Bengni u-šo: (Sun } \\
\text { 1993: 93) }\end{array}$ \\
\hline chicken & $\begin{array}{l}\# 1604 \text { *daw OR *dow } \\
\text { BIRD }\end{array}$ & do: & $\mathrm{do}^{\mathrm{u}}$ & $\begin{array}{l}\text { dok do? } \\
\text { dup }\end{array}$ & d3uo & *dowC & - \\
\hline child & $\begin{array}{l}\# 2708 * m-(t / d) u \\
\text { NEPHEW/ DESC. }\end{array}$ & məন্ধu: & - & amai (SM) & sa (SA) & - & TS za 'son' \\
\hline chin & - & məgu: & gut $\int a(?)$ & gut $\int a(?)$ & $\begin{array}{l}\text { Pĕgado; gaḑo } \\
\text { (SA) }\end{array}$ & *mə-ga-da? & $\begin{array}{l}\text { Koro gumdu (AK); PK } \\
\text { akou; BG } t^{\text {h}} \partial k^{\text {h}} a u ; K D, \\
\text { SS gamde; DS } \\
\text { gamt }^{\text {h } u \eta}\end{array}$ \\
\hline cloth & - & gì? & gije & gije gəri grə & gije & *gi-le & Koro gile (AK) \\
\hline cloud & - & wa:wə: & $\begin{array}{l}\text { ma'man } \\
\text { ma'mər }\end{array}$ & $\begin{array}{l}\text { miemy mimu; } \\
\text { meimiu (SM) }\end{array}$ & $\begin{array}{l}\text { məmə; } \\
\text { mum(u) (SA) }\end{array}$ & *majməwn & $\begin{array}{l}\text { PKC *may; PT } \\
{ }^{*} \mathrm{~m}(ə / \mathrm{u}) \mathrm{k}\end{array}$ \\
\hline coal & - & $\begin{array}{l}\text { mje:pe:zin } \\
\text { me:pəzin }\end{array}$ & - & - & - & - & - \\
\hline cook & - & ku: & $k \partial \sim k^{h} u$ & $k^{h} \partial \sim k^{h} u$ & $k^{h} u ; k^{h} o(S A)$ & ${ }^{*} \mathrm{ku}$ & - \\
\hline cooking oil & - & 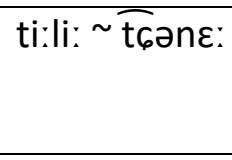 & pandzanvə & $\begin{array}{l}\text { p }^{\text {hendzanrz; }} \\
\text { p enzanvu }^{\text {henza) }} \\
\text { (SM) }\end{array}$ & luboxu & - & IA tēl \\
\hline copula & $\begin{array}{l}\text { \#450 *way BE / } \\
\text { COPULA }\end{array}$ & wi & - & - & - & - & - \\
\hline cow & - & so: & Jufə & $\begin{array}{l}\text { Jufu dzufu } \\
\text { dərfu }\end{array}$ & $\begin{array}{l}p^{\text {h}} \text { olxu; fulxu } \\
\text { (SA) }\end{array}$ & $*$ su & PTk *se; KD $6 a$ \\
\hline cry & \#1103 *krap WEEP & $k(j) e:$ & krəmna & $k^{\text {h̆ }}$ & $k \int 0$ & $*_{\mathrm{kraC}}$ & $\begin{array}{l}\text { OC *k-rəp; TS gep; KD } \\
k^{\text {hip }}\end{array}$ \\
\hline cut & & $\mathrm{t} \varepsilon \sim \mathrm{tæ}$ & $\begin{array}{l}\text { ta'na rap } \\
\text { voăna }\end{array}$ & $\mathrm{t}^{\mathrm{h}} \mathrm{a}^{\mathrm{i}}(\mathrm{ne})$ & dzojo; $\widehat{d} \xi \varepsilon(S A)$ & $*_{\text {taj }}$ & $\begin{array}{l}\text { PTk *tat; PT *tək CUT } \\
\text { UP; TS tok }\end{array}$ \\
\hline day & - & agu: & gijantan & dzantan hũe & jo & *ga & - \\
\hline
\end{tabular}




\begin{tabular}{|c|c|c|c|c|c|c|c|}
\hline Gloss & PTB & Bangru & E. Miji & W. Miji & Hruso & $\mathrm{PH}$ & $\begin{array}{l}\text { Proto-forms, } \\
\text { isoglosses }\end{array}$ \\
\hline dead body & $\begin{array}{l}\# 17 \text { *s-man BODY / } \\
\text { CORPSE }\end{array}$ & səmo: & - & - & $\overline{\mathrm{dz} u}(\mathrm{SA})$ & - & $\begin{array}{l}\text { PT *si-man; Puroik } \\
\text { səma: }\end{array}$ \\
\hline deep & - & mərju: & mərə & $\begin{array}{l}\text { arək məru? } \\
\text { merik }\end{array}$ & rju & *mə-ruk & $\begin{array}{l}\text { PT * run; PKC * ruak } \\
\text { EMPTY; SS 'aruk } \\
\text { 'empty' }\end{array}$ \\
\hline die & $\# 27$ *səy & tai & teina & $t \int i$ & $\begin{array}{l}\text { dzudzo; dza/i } \\
\text { (SA) }\end{array}$ & *Өəј & PKC *thii-I, thi?-II \\
\hline dig & $\# 3573$ *s/m-du & ta: & - & $\mathrm{t}^{\mathrm{h}} \underset{v}{\mathrm{u}}(\mathrm{SM})$ & $\mathrm{t} \int \mathrm{i}(\mathrm{SA})$ & $* \operatorname{taC}$ & - \\
\hline dirty & - & nətढ़i & - & nit $\int \mathrm{i}(\mathrm{SM})$ & nits kriniu (SA) & $*$ ni-t $\bar{i} \mathrm{i}$ & - \\
\hline do & - & ru: & rəna $\sim$ ru & $\begin{array}{l}\text { ru } \sim \text { rut } \int i n e ~ \\
t^{\text {herore }}\end{array}$ & dad3o & *ru & $\begin{array}{l}\text { PT *rju; PNN *rə:y; } \\
\text { BG rjet; SS re(t/P) } \\
\text { ra(t/P); KD le; } \\
\text { Nyasang ri; Bengni rji }\end{array}$ \\
\hline door & - & nepãĩ & banpi vaphi & $\begin{array}{l}\operatorname{vimp}^{h_{i}} \sim \operatorname{vap}^{h} i \\
\text { banp }^{\text {hik }}\end{array}$ & $\begin{array}{l}\text { nĕgdə]e } \\
\text { nekdzusi (SA) }\end{array}$ & *pin & BG ha:pin \\
\hline dream & $\# 130$ *r/s-mwəy & tjamə: & $\begin{array}{l}\text { ta'mat } \\
\text { ta'məna }\end{array}$ & $\begin{array}{l}t^{\text {h }} \text { eme }(\mathrm{ma}) \sim \\
\mathrm{t}^{\text {hal }} \mathrm{a}^{\mathrm{i}} \text { ? }\end{array}$ & $\mathrm{t} \mathrm{h}^{\mathrm{h}}$ imijo & $*_{\text {tai-mə }}$ & OC*C.mən-s \\
\hline drink & $\# 502$ *N/s-tun & ton $\sim$ tun & $\mathrm{t}^{\mathrm{h}}$ on $\sim$ topna & $\begin{array}{l}t^{\text {hoo }} \sim t^{\text {hoonme; }} \\
t^{\text {h }} \text { un (SM) }\end{array}$ & $t^{\text {thudzo }}$ & $*$ tun & WT 'athung \\
\hline dry & - & məki: & mek $^{h} i j a n$ & $\begin{array}{l}\text { ak }^{h} \text { ijan } \\
\text { mək } \\
\text { mek }^{h} \text { ijam; } \\
\text { mək }^{\text {hjan }} \text { (SM) }\end{array}$ & kıo; knrou (SA) & *mə-ki-lan & BG əkou \\
\hline eat & $\# 36 * N-d z(y) a-k / n / t / s$ & t6 $\dot{u}^{2} \sim \widehat{t 6} \partial^{2}$ & $\mathrm{t} \int \mathrm{u} \sim \mathrm{t} \int ə n a$ & $\mathrm{t} \int \mathrm{u} ; \mathrm{tsu}$ (SM) & 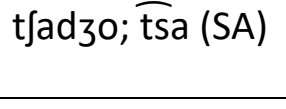 & $* \widehat{t 6 a}$ & 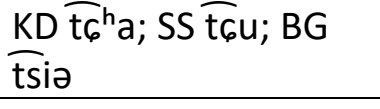 \\
\hline egg & $\# 3438$ * $(r / I)$ um & do:rein & dorin doriet & dori(n) & dzedze & *do-rin & BG əri: \\
\hline eight & \#2259 b-r-gyat & səgài? & 6əgə & t]igə səgə & $\begin{array}{l}\int \partial g d 3 \partial ; \text { sə⿹𠃌口 } \\
\text { (SA) }\end{array}$ & *sə-giC & SS, KD sarg $\varepsilon$ \\
\hline
\end{tabular}




\begin{tabular}{|c|c|c|c|c|c|c|c|}
\hline Gloss & PTB & Bangru & E. Miji & W. Miji & Hruso & $\mathrm{PH}$ & $\begin{array}{l}\text { Proto-forms, } \\
\text { isoglosses }\end{array}$ \\
\hline elder brother & \#2362 * (g/k)əw-n & ako: & kəvoi & $\begin{array}{l}\text { akh }^{\text {həvo } ~ a k \partial v o ~} \\
\sim k^{h} \text { əvo? }\end{array}$ & $\begin{array}{l}\text { Paja; á-khī } \\
\text { (elder), ắ-yă } \\
\text { (younger, AS) }\end{array}$ & *(a/mə)ko & BG k ${ }^{\mathrm{h}}$ ua (younger) \\
\hline elephant & - & səte: sətə: & atai & at $\int \mathrm{i}$ & at $\int^{\mathrm{h} \partial}$ & - & IA hāt ${ }^{h_{i}}$ \\
\hline empty & - & məhe: (ruaroy) & mihijan & $(a / m i) k^{h} i j a \eta$ & $\begin{array}{l}\text { Рəфо; ufou } \\
\text { (SA) }\end{array}$ & *mə-hi-lan & $\begin{array}{l}\text { PNC *hoom EMPTY / } \\
\text { VACANT }\end{array}$ \\
\hline $\begin{array}{l}\text { excrete, } \\
\text { defecate }\end{array}$ & - & (lai) ţai & - & - & $\widehat{\text { tsi }(S A)}$ & * tşaj & $\begin{array}{l}\text { PKC *tse? URINATE / } \\
\text { DEFECATE; TS, KD } \\
\frac{\mathrm{t} \int^{\mathrm{h}} \mathrm{Et} ; \text { Bugun tse:; SS }}{\mathrm{t} \mathrm{h}^{\mathrm{h}} \mathrm{a}:}\end{array}$ \\
\hline excrement & $\begin{array}{l}\text { \#572 *kləy BODY DIRT } \\
\text { / EXCREMENT }\end{array}$ & lai & - & - & - & - & - \\
\hline eye & \#33 *s-myak そ s-mik & mejà? & mẽ & $\begin{array}{l}\text { məri me? } \\
\text { mer }\end{array}$ & Pini & $*_{\text {mə-ja? }}$ & BG mjहk \\
\hline far & $\begin{array}{l}\# 1011 *^{*} \mathrm{~s}-\mathrm{r}(\mathrm{i} / \mathrm{u}) \eta \\
\text { LONG }\end{array}$ & arən & mərən & $\begin{array}{l}\text { maran arən } ~ \\
\text { mərən }\end{array}$ & Рәхә; әrа (SA) & *mə-rəり & $\begin{array}{l}\text { BG ruan 'far'; KD } \\
\text { Purin Pulin; SS } \\
\text { Paren; TS ripbu all } \\
\text { 'long' }\end{array}$ \\
\hline fat (n) & $\begin{array}{l}\# 163{ }^{*} \mathrm{~s}-\mathrm{b}^{\mathrm{w}} \mathrm{a}(\mathrm{m} / \mathrm{p}) \\
\text { SWOLLEN/FAT/THICK }\end{array}$ & məba: & $\begin{array}{l}\text { məmbau } \\
\text { məmbou }\end{array}$ & membau & Păbi & *mə-baC & - \\
\hline fear & \#2337 *kri(y) & nirimè & - & $\operatorname{rin}(\mathrm{SM})$ & rije (SA) & $*$ ri & BG ry:m \\
\hline few & - & mi:gəme & mija & me? mija & me? & ${ }^{*}$ mej? & - \\
\hline fight & \#2596 *ray & ware: & - & nau-ri (SM) & $\mathrm{t}^{\mathrm{h}} \mathrm{o}-\mathrm{ri}(\mathrm{SA})$ & *rəj & - \\
\hline finger & $\# 330 *(t) s(y) o w$ & məgعitढంa & gitfo? ditfo & $\begin{array}{l}\text { gitjo? git }{ }^{\mathrm{h}} \text {; } \\
\text { gitso (SM) }\end{array}$ & $\begin{array}{l}\text { Pĕdzət } \int \partial ; \\
\text { egzətsə (SA) }\end{array}$ & ${ }^{*}$ mə-guC-t6o? & - \\
\hline fire & \#2136 *mey & $m \varepsilon:$ & mai & mai & $\mathrm{mi}$ & ${ }^{*}$ maj & - \\
\hline fireplace & \#2599 *g-rap & là? & - & dət $\mathrm{t}^{\mathrm{h}} \mathrm{le}$ (SM) & huk $^{\text {hije }(S A)}$ & *laC & - \\
\hline
\end{tabular}




\begin{tabular}{|c|c|c|c|c|c|c|c|}
\hline Gloss & PTB & Bangru & E. Miji & W. Miji & Hruso & $\mathrm{PH}$ & $\begin{array}{l}\text { Proto-forms, } \\
\text { isoglosses }\end{array}$ \\
\hline fish & - & tsədzome: & $\mathrm{t} \int \mathrm{o}^{\mathrm{i}}$ & $\begin{array}{l}t^{\mathrm{h}}(r) i ; t^{\mathrm{h}} u i \sim \widetilde{\mathrm{t} J u i} \\
(\mathrm{SM})\end{array}$ & $t \int^{h} e ; t^{h} \int^{h} i(S A)$ & $* \operatorname{trV}$ & $\begin{array}{l}\text { Guiqiong tJə55 ni55 } \\
\text { (TBL); Mawo Qiang } \\
\text { вzə (TBL) }\end{array}$ \\
\hline five & \#1306 *l/b-na & рәрu: & punu & $\begin{array}{l}\text { bonu bunu } ~ \\
\text { pugu }\end{array}$ & pom; pum (SA) & *bə-пu & PCN *pha-na \\
\hline flow & $\begin{array}{l}\# 2680 \text { *sywar } \Varangle \\
* g / b-s y w a-n / t\end{array}$ & sa: & - & - & - & - & - \\
\hline flower & \#2185 *b/s-wat & məbua: & gəbo & ubo? & Joba & *ma-boC & PK məbuai?; BG əbua \\
\hline friend & - & məন্dzua & mad3o & $\begin{array}{l}\operatorname{məd} 30 \sim \\
\operatorname{mad} 3 r o \sim \operatorname{ad} 30\end{array}$ & Pod30; $\widehat{d} \xi \varepsilon(S A)$ & 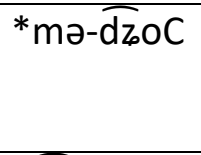 & $\begin{array}{l}\text { PT *`(o/u)n *jen; } \\
\text { Pur. a-dua ; BG } \\
\text { e:dzak }\end{array}$ \\
\hline frog & $\# 645 * \mathrm{t}(\mathrm{u} / \mathrm{i}) \mathrm{k}$ & $\overline{d z} \partial:$ & dzou & dzou & Jedza; Јәza (SA) & $* \overline{\mathrm{d} z} \mathrm{u} \mathrm{C}$ & - \\
\hline fruit & $\# 1019$ *sey & mət $\tilde{\varepsilon}:$ & gutan & $u t^{\text {hen }}$ & Jə̆dze & *Oai & PKC *thay \\
\hline four & \#2409 *b-Іәу & puruwẽ & pəlei & bəlei bli & 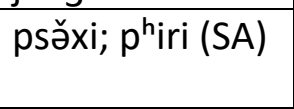 & *bə-ləj & $\begin{array}{l}\text { TM bli; TS, KD p६i; SS } \\
\text { bəsi; PLB *b/P-ləy }{ }^{2}\end{array}$ \\
\hline full & $\# 111 *(p / b)$ lin & ləクbəla & landay len & 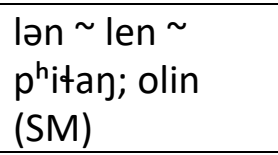 & gu & *lin & BG əlije: \\
\hline garlic, onion & - & jək ع̃̃ & kiomu kemon & 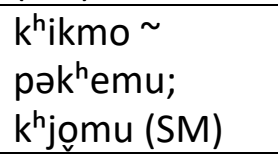 & sukum (SA) & *kikmun & $\begin{array}{l}\text { KD mon; BG muan; SS } \\
\text { monsin }\end{array}$ \\
\hline give & \#2158 *s-bəy-n/k & bai & $b^{i} \sim b^{i} n a$ & $\begin{array}{l}p^{\text {hebi }} \sim \text { bi?na } \\
\text { bi }\end{array}$ & dzid3o & *bəj & $\begin{array}{l}\text { TS, TM bi; KD ढi; BG } \\
p^{\text {hy }} ; \text { PLB * bəy }{ }^{2}\end{array}$ \\
\hline goat & \#6033 *bi-n & səp & $\begin{array}{l}\text { Japen } \sim \\
\text { suprem }\end{array}$ & 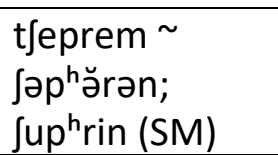 & $k^{h} \partial \int \partial ; k^{h} i s i(S A)$ & *se-preN & $\begin{array}{l}\text { BG səphin; PBG } \\
\text { prunn }^{2}\end{array}$ \\
\hline grandfather & $\begin{array}{l}\# 1638 \text { *b/m-lan PENIS } \\
\text { / MALE / HUSBAND }\end{array}$ & məlo: alo: & - & alu (SM) & - & - & - \\
\hline
\end{tabular}




\begin{tabular}{|c|c|c|c|c|c|c|c|}
\hline Gloss & PTB & Bangru & E. Miji & W. Miji & Hruso & $\mathrm{PH}$ & $\begin{array}{l}\text { Proto-forms, } \\
\text { isoglosses }\end{array}$ \\
\hline grandmother & $\begin{array}{l}\text { \#2665 *sru(w) AUNT/ } \\
\text { ELDER SISTER }\end{array}$ & ase: məse: & - & azui (SM) & - & - & WT sro-mo 'aunt' \\
\hline grass & $\begin{array}{l}\text { \#5642 *s-yəy GRASS / } \\
\text { WEEDS }\end{array}$ & $\begin{array}{l}\text { rəsja:re: } \\
\text { rəse:re: }\end{array}$ & - & tsen (SM) & $\begin{array}{l}\text { bije } \widetilde{\text { tsə } \sim} \\
\text { suso (SA) }\end{array}$ & - & - \\
\hline grind, crush & \#2333 * kri:t & rài & - & ri (SM) & ri (SA) & *rajC & PT * rit \\
\hline guts & \#2116 *r-gyu-n (prov.) & mələgu: & - & Iun (SM) & - & ${ }^{*}$ mə-lun & BG əlui \\
\hline hair & $\begin{array}{l}\text { \#1228 *s-pu FEATHER } \\
\text { / WING / HAIR (body) }\end{array}$ & gápł̀ & gopal gopə & $u^{h_{i} \mathrm{i}^{\mathrm{u}}} \sim$ wop $^{\mathrm{h}_{\mathrm{ijj}}}$ & 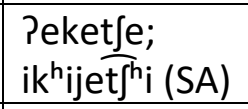 & *go-pu & WT mgo-spu \\
\hline hard & \#174 *s-ra & məlo: & - & məgan (SM) & $\begin{array}{l}\text { goyou (AA); } \\
\text { gorou }\end{array}$ & *mə-gan-lo & - \\
\hline hammer & - & martolə & tృmpo & $\begin{array}{l}\text { tJampo; } \\
\text { tsimp }\end{array}$ & martăl & - & IA martal \\
\hline hand, arm & $\# 712 * k(r) u-t$ & məgغ̀ं? & gi & gi & $\begin{array}{l}\text { Pagd3ə; e egz } \\
\text { (SA) }\end{array}$ & $*_{\text {mə-guC }}$ & - \\
\hline have; exist & \#3602 *du STAY/ LIVE & du: & - & $\mathrm{du}(\mathrm{SM})$ & du (SA) & $* d u$ & WT 'dug \\
\hline head & $\begin{array}{l}\# 386 \text { *m-gaw \ s- } \\
\text { gaw; \#1224 *kuk }\end{array}$ & go:kẼĩn & $(\mathrm{mo}) \mathrm{go}^{\mathrm{u}}$ & $\begin{array}{l}\text { dək }{ }^{\text {hijan }} \sim \mathrm{u}: ~ \\
\text { tokijan }\end{array}$ & Pek'je & *mə-go-kun & - \\
\hline heart & $\# 1381 * m-\operatorname{lu}(\mathrm{n} / \mathrm{m})$ & malonwə: & lonvə lunvəu $\mathrm{k}$ & $\begin{array}{l}\text { lunvəuk } \sim \\
\text { kebanlan } \\
\text { məjijo }\end{array}$ & Pălăbiu & *mə-lun-wəwC & BG ələbau \\
\hline heavy & $\# 2415$ *s-(l/r)əу-t & latəク & məle & $\begin{array}{l}\text { məji məi? } \\
\text { ai }\end{array}$ & ju; liu (SA) & *mə-ləj & $\begin{array}{l}\text { BG əlai; SS Pali; KD } \\
\text { Puli }\end{array}$ \\
\hline horn & - & masu & (məsə)zun/Jon & $\begin{array}{l}(\mathrm{m} / \mathrm{a})\left(\int \mathrm{u} / \mathrm{Si}\right) \\
\text { Jon } / \mathrm{d} z u n\end{array}$ & $\begin{array}{l}\text { РəЈəbdza; } \\
\text { səБ̄zzə (SA) }\end{array}$ & *mə-su & - \\
\hline horse & - & go:ra: & - & Jugro (SM) & fugra (SA) & - & IA g odā \\
\hline house & $\begin{array}{l}\text { \#5746 * nam VILLAGE } \\
\text { (+HOUSE (prop.)) }\end{array}$ & ne: & nam & $\begin{array}{l}\text { nan ne } \\
\text { neon; nen }(S M)\end{array}$ & ne & *nam & $\begin{array}{l}\text { Galo namə (GLDC } 09 \\
\text { Galo); Koro ne (AK); } \\
\text { PBG nok; Baram nam } \\
\text { (Kansakar 2010) }\end{array}$ \\
\hline
\end{tabular}




\begin{tabular}{|c|c|c|c|c|c|c|c|}
\hline Gloss & PTB & Bangru & E. Miji & W. Miji & Hruso & $\mathrm{PH}$ & $\begin{array}{l}\text { Proto-forms, } \\
\text { isoglosses }\end{array}$ \\
\hline how many & - & kənəru: & $k^{\mathrm{h}}$ әла & (kə/gə)no & $k^{\mathrm{h}}$ әла & *kə-na & - \\
\hline human & $\begin{array}{l}\# 1002 * r-m i(y)-n \\
\text { PERSON/MAN }\end{array}$ & ləwə: nəwə: & nijə & niu?; ni (SM) & năna & $*_{\text {niC }}$ & - \\
\hline hundred & - & ləク & palon & blun belun & $\begin{array}{l}\text { фoxa; } p^{\text {h}} \text { uyu } \\
\text { (SA) }\end{array}$ & *bə-lun & $\begin{array}{l}\text { PT *Iun; Koro pələ } \\
\text { (AK) }\end{array}$ \\
\hline ill & $\begin{array}{l}\# 160 \text { *na-(n/t) ILL/ } \\
\text { PAIN/SORE/ ACHE }\end{array}$ & nua $\sim$ noa & - & no (SM) & na (SA) & *noC & - \\
\hline insect & $\begin{array}{l}\text { \#2178 *bəw INSECT/ } \\
\text { SNAKE }\end{array}$ & bə:lon & bilo bilun & bilun & bŭl:u; bilu (SA) & *bəw-lun & - \\
\hline iron & \#2676 *syam & sə: & - & sen (SM) & so (SA) & *suN & - \\
\hline itch & $\begin{array}{l}\# 199 \text { *m-tsik; \#1457 } \\
{ }^{*} \text { m-sak }\end{array}$ & kəव̆dzù? & - & gudzu (SM) & sə⿳亠口冋u (SA) & 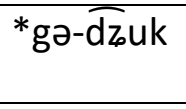 & - \\
\hline kick & - & pa: & təgenna & $\begin{array}{l}\text { laitegen } \\
\text { dəgrən } \\
\text { tekran }\end{array}$ & $\begin{array}{l}\text { gədrə; gəḑ̄u } \\
\text { (SA) }\end{array}$ & *dəgran & - \\
\hline kill & - & gja: & gaina & $\begin{array}{l}\text { wai waitjo } \\
\text { (strike, beat?) }\end{array}$ & gə (SA) & *gajC & - \\
\hline knife & - & we:tsəク & vaitfonzi & 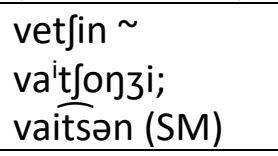 & $\begin{array}{l}\text { betJasa; vetsə } \\
\text { (SA) }\end{array}$ & *vaj-tsun & PKC *tsem \\
\hline know & - & ni: & nina nı̆ & 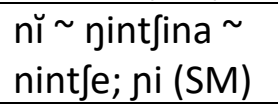 & $\begin{array}{l}\text { dayxudzo; } \\
\text { hosə (SA) }\end{array}$ & $*_{n i}$ & - \\
\hline laugh & - & tua & tona & $\mathrm{t}^{\mathrm{h}} \mathrm{O} \sim \mathrm{t}^{\mathrm{h}} \mathrm{O}^{\mathrm{u}}$ & $\mathrm{t} \mathrm{f}^{\mathrm{h}}$ әjо; $\widehat{\mathrm{t} f \mathrm{i}}(\mathrm{SA})$ & *toC & - \\
\hline leaf & $\begin{array}{l}\# 2085 \text { *rwak; \#824 } \\
\text { *s-lap }\end{array}$ & mərjì ${ }^{?}$ & məlam golap & məle ule? & Pə̆xe; Jere (SA) & ${ }^{*}$ mə-rajC & $\begin{array}{l}\text { BG ərap; KD Pulap, SS } \\
\text { Pale: }\end{array}$ \\
\hline leech & $\# 2555$ *k-r-pwat & ləwغे & - & dəve (SM) & - & *Iə-wajC & SS p phys: \\
\hline leg & \#350 *la LEG/FOOT & məle: & lei & lai & əfi ləbu (SA) & *laj & KD lej; SS Iع: \\
\hline lick & $\begin{array}{l}\text { \#628 *s-lya:w LICK / } \\
\text { TONGUE }\end{array}$ & kəlo: & - & - & - & - & BG ljak; KD lak \\
\hline
\end{tabular}




\begin{tabular}{|c|c|c|c|c|c|c|c|}
\hline Gloss & PTB & Bangru & E. Miji & W. Miji & Hruso & $\mathrm{PH}$ & $\begin{array}{l}\text { Proto-forms, } \\
\text { isoglosses }\end{array}$ \\
\hline lift & \#5406 *lan & Iõ(n) & jan ijanna & jan Jan & lo (SA) & *lan & - \\
\hline light & - & lətõ: & mənətan & $\begin{array}{l}\text { malət }{ }^{\text {han }} \sim \\
\text { alithan }^{\sim} \\
\text { mədət }^{\text {han }}\end{array}$ & $\begin{array}{l}\text { lăto; lut }{ }^{\text {hou }} \\
\text { (SA) }\end{array}$ & *mə-lə-tan & BG $\partial t^{\mathrm{h}} \mathrm{Ou}$ \\
\hline liquor & - & $\bar{t} \overline{t i}^{2}$ & (pə̆)t $\int a \eta$ & t Jan & $\mathrm{t} \int \partial ; \widehat{\mathrm{tsi}}(\mathrm{SA})$ & $* \overline{t 6 i}$ & WT chang \\
\hline listen, hear & \#811 *r-gna & rei & roina & rei & $\begin{array}{l}\text { dzədzo; dzəxu } \\
\text { (SA) }\end{array}$ & *rej & - \\
\hline live, grow up & \#71 *s-rin \ s-r(y)an & səy & - & Sən (SM) & әЈә (SA) & $* \sin$ & NY sən; OC *sren \\
\hline liver/lungs & $\begin{array}{l}\# 1390 * m-\sin \text { LIVER/ } \\
\text { HEART/BILE }\end{array}$ & 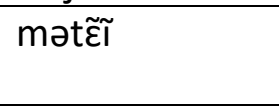 & - & mət $^{h}$ ən (SM) & ě-djǔ (AS) & ${ }^{*}$ mə-Oin LIVER & $\begin{array}{l}\text { PCN *m-sən; PKC } \\
\text { *thin LIVER }\end{array}$ \\
\hline long & - & $\begin{array}{l}\text { məpan; mə31 } \\
\text { bran }^{55}(\text { LD) }\end{array}$ & məpijan & məpijan & pfu; psiu (SA) & *mə-pją & 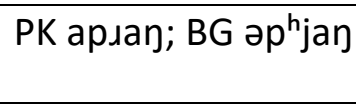 \\
\hline look, see & $\begin{array}{l}\# 1405 * \text { kan } \\
\text { KNOW/SEE }\end{array}$ & gõ: & ganna & wan & hod3o & *gan & PT *kan \\
\hline louse & $\begin{array}{l}\# 2652 \text { *s(y)ar; \#2609 } \\
\text { *s-r(y)ik }\end{array}$ & sà? & - & fi (SM) & Јə (SA) & $* \mathrm{saC}$ & - \\
\hline make; do & \#552 * day DO & $\mathrm{da} \sim \mathrm{d} \varepsilon:$ & - & - & dad3o & $*$ daj & - \\
\hline man (male) & $\begin{array}{l}\# 1002 * r-m i(y)-n \\
\text { PERSON / MAN; } \\
\text { \#5484 *wa MAN/ } \\
\text { PERSON }\end{array}$ & nəwi: & nәјә & $\begin{array}{l}\text { niju nu? } \\
\text { nuvə; nuvu } \\
\text { (SM) }\end{array}$ & $\begin{array}{l}\text { mə̆hu; muxu } \\
\text { nəna (SA) }\end{array}$ & $*_{\text {niC }}$ & - \\
\hline meat & \#34 *sya-n & sù? $6 \mathrm{U}^{3}$ & sikijun & Jitfun & фu; tsə, sə (SA) & $*$ su & $\begin{array}{l}\text { PKC * } s^{h} \text { aa ANIMAL / } \\
\text { FLESH / MEAT }\end{array}$ \\
\hline medicine & - & do:wai & dawa & dawa & doxa & - & IA davā \\
\hline melt & - & nua gua; $\widehat{d z} \varepsilon:$ & - & - & - & - & PT * jit $~ *$ ǰet \\
\hline month & $\begin{array}{l}\# 1016 * \text { *-(g)la } \\
\text { MOON/MONTH }\end{array}$ & lù? & la & lə lu & $k^{h}$ obe & $* l u$ & - \\
\hline moon & -do- & alo: ləbai & la & $\mid \varepsilon \sim \mathrm{lu}$ & $\begin{array}{l}\text { hŏbe; hubje } \\
\text { (SA) }\end{array}$ & $* \operatorname{lu}$ & - \\
\hline
\end{tabular}




\begin{tabular}{|c|c|c|c|c|c|c|c|}
\hline Gloss & PTB & Bangru & E. Miji & W. Miji & Hruso & $\mathrm{PH}$ & $\begin{array}{l}\text { Proto-forms, } \\
\text { isoglosses }\end{array}$ \\
\hline mountain & $\begin{array}{l}\text { \#3581*s-gan } \\
\text { MOUNTAIN/ RIDGE, } \\
\text { HILL }\end{array}$ & gan səpi: & pon & $p^{\text {h}} u n \sim p o n$ & $\begin{array}{l}p^{h} u ; p^{h} u d e u \\
\text { (SA) }\end{array}$ & - & $\begin{array}{l}\text { Koro gon (AK); PK } \\
\text { gגan; WT sgang; } \\
\text { Miji/Hruso: PTk } \\
{ }^{*} p^{h} \text { un }\end{array}$ \\
\hline $\begin{array}{l}\text { mouth; } \\
\text { language }\end{array}$ & - & məләฤ & nek nelə ${ }^{i}$ & niwi $\sim$ nui & $\begin{array}{l}\text { Pănzu; undzu } \\
\text { (SA) }\end{array}$ & *mə-nun & $\mathrm{PCN} *$ mən \\
\hline mortar & - & lara: & - & dəlo (SM) & rulu (SA) & $*$ Iu-IV & TS lu \\
\hline mother & $\begin{array}{l}2507 \text { *ney } ð \text { ni(y) } \\
\text { AUNT/ MOTHER-IN- } \\
\text { LAW }\end{array}$ & məлє: ane: & ane & anai $\sim$ ani & ani & $*_{\text {me-naj }}$ & $\begin{array}{l}\text { PKC *nii; PTk *ni } \\
\text { AUNT }\end{array}$ \\
\hline name & $\# 2450 *_{r-m i(\eta / n)}$ & məmjəワ & mãrn & mãrn & $\begin{array}{l}\text { Pəләлә; Panini } \\
\text { (SA) }\end{array}$ & ${ }^{*}$ mə-mjin & TM mjen \\
\hline near & \#2496 *s-ney & məre: & məroi & məni anin & Pənisə & ${ }^{*}$ mə-nej & - \\
\hline neg. imp. & $\# 2681 *(t / d) a$ & tu- & - & $t^{\mathrm{h}} \mathrm{a}-(\mathrm{SM})$ & $\widehat{d z} u-(S A)$ & *ta- & $\begin{array}{l}\text { SS thə-; KD tha-; BG } \\
\text { də- }\end{array}$ \\
\hline new & - & məkərə: & məkərə & $\begin{array}{l}\text { məgəniu } \\
\text { agənu }\end{array}$ & $\begin{array}{l}\text { Păkə̆n; ək } \\
\text { (SA) }\end{array}$ & *mə-gə-nu & - \\
\hline night & - & nəga: aga: & (k/g)engau & (dzan/tJan)gau & negi & ${ }^{*}$ nə-gaC & - \\
\hline nine & - & sətəク & 6ətan & sətən & sțə; st ${ }^{\mathrm{h}} \partial$ (SA) & *sə-tin & - \\
\hline nose & \#2093 *ka そ* * па & $\begin{array}{l}\text { miko: } \\
\text { mənəko: }\end{array}$ & nebijon & $\begin{array}{l}\text { nibun } \sim \text { nibiun } \\
\sim \text { nebijon } \\
\end{array}$ & $\begin{array}{l}\text { Pufə; (un/nu) } \\
\text { su (SA) }\end{array}$ & - & - \\
\hline old & - & məяua: & məJo? & (a/mə) Jo? & $\begin{array}{l}\text { Păme; emije } \\
\text { (SA) }\end{array}$ & $*$ mə-60? & - \\
\hline one & - & akã: akí: & $\begin{array}{l}\text { an ak }{ }^{\text {hijo }} \sim \\
\text { ak }{ }^{\text {h}} \text { owa }\end{array}$ & $\begin{array}{l}a k^{h} e ? \sim a k^{h} i j o \sim \\
a k^{h} o \sim \text { atro }\end{array}$ & $\mathrm{Pa}$ & *a-ken & PT *kon \\
\hline otter & \#2595 *s-ram & sẽ: & - & - & size (SA) & - & WT sram \\
\hline paddy rice & - & $\tilde{\varepsilon}:$ & nam & $\begin{array}{l}\text { enfo anso? } \\
\text { an }\end{array}$ & ?o & *an & $\begin{array}{l}\text { PT *am-bun RICE } \\
\text { (UNCOOKED) }\end{array}$ \\
\hline penis & \#1624 *la MALE & məlò? & - & - & - & - & KD, SS, BG lak \\
\hline
\end{tabular}




\begin{tabular}{|c|c|c|c|c|c|c|c|}
\hline Gloss & PTB & Bangru & E. Miji & W. Miji & Hruso & $\mathrm{PH}$ & $\begin{array}{l}\text { Proto-forms, } \\
\text { isoglosses }\end{array}$ \\
\hline pestle & - & pəla: & - & bəlau (SM) & - & *bə-laC & - \\
\hline pig & \#1006 *pwak & jù? & d3o(?) & $\begin{array}{l}\text { dzio dzuo } \\
\text { dzo?; } 30 \text { (SM) }\end{array}$ & vo; vo (SA) & *jow? & $\begin{array}{l}\text { PKC *wok; PTk } \\
\text { *hwok; BG wok }\end{array}$ \\
\hline pillow & $\# 692 * m-k(u / i) m$ & gokẽ & gokəm & $\begin{array}{l}u^{\text {h }} u m \sim \\
\text { (nu/wo)k }\end{array}$ & dĭkjo & *go-kuN & - \\
\hline pull & - & sa: & bre Gaana & ro? horo & byjo & - & - \\
\hline put & - & wu: vu: & - & rou (SM) & - & - & - \\
\hline rain & $\begin{array}{l}\# 298 * m-t(w) ə y-n \Varangle \\
* m-t i-s \text { WATER etc. }\end{array}$ & nədi: & fro & fro; $p^{h}$ rjo (SM) & $\begin{array}{l}\text { jedzăfə; } \\
\text { ne⿳亠dzəJi (SA) }\end{array}$ & *nə-dəj; *pro & 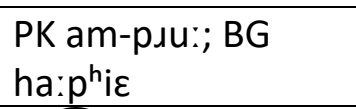 \\
\hline red & 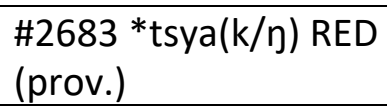 & jaţò? & $\operatorname{mot} \int i$ & $\begin{array}{l}\text { mət } \int \partial \sim \operatorname{mat} \int u \\
\sim \text { atfu }\end{array}$ & $\mathrm{t} \int \mathrm{u}$ & *tढa? & TS tsalu \\
\hline revenge & - & ra: & - & - & - & - & - \\
\hline ring & - & gəle? & gilan & gile $\sim$ gilan & $\begin{array}{l}\text { (gzə⿹tsə/dzว̆tse) } \\
\text { lje }\end{array}$ & *gə-leC & Koro lale (AK) \\
\hline river & $\begin{array}{l}\text { \#298*m-t(w)əy-n } \\
\text { WATER / RIVER; } \\
\text { \#2322 *kI(y)u(y/k) } \\
\text { VALLEY / RIVER }\end{array}$ & wəgo: & vəją vədo & vəją vədo & $k^{h} u ; x u d \varepsilon u(S A)$ & - & PTk *kon \\
\hline road & \#1017 *lam & re:bõ & laban & laban lemban & xabo; rabo (SA) & *lam-ban & $\begin{array}{l}\text { Apatani lem-bo (PT) } \\
\text { Chungli Ao lə } 1 \text { man } \\
\text { (PCN); TM lemdan }\end{array}$ \\
\hline root & - & məkẽ̃̃ məltù & $\begin{array}{l}\text { mək }{ }^{\text {hən } ~} \\
\text { gok }^{\text {hran }}\end{array}$ & $\begin{array}{l}k^{h} \text { rən } \sim \text { ok }^{h} \text { rin } \\
\sim \text { uk }^{\text {h }}\end{array}$ & $\begin{array}{l}\text { PăkJe; ək }{ }^{\mathrm{h}} r \partial \\
\text { (SA) }\end{array}$ & *mə-krin & BG ərə⿹ \\
\hline round & - & məwə: & mədəriu & mədəriu & $\begin{array}{l}\text { odzou; } \\
\text { udzərəu (SA) }\end{array}$ & *mə-də-rəw & - \\
\hline rub & $\begin{array}{l}\text { \#2674 *sywəy RUB / } \\
\text { SCRAPE / SHAVE }\end{array}$ & pəsci & - & - & - & - & - \\
\hline run & \#6163 *b-(l/r)əy & bi: & - & 3um (SM) & - & - & - \\
\hline sago palm & & lowo: & - & - & - & - & - \\
\hline
\end{tabular}




\begin{tabular}{|c|c|c|c|c|c|c|c|}
\hline Gloss & PTB & Bangru & E. Miji & W. Miji & Hruso & $\mathrm{PH}$ & $\begin{array}{l}\text { Proto-forms, } \\
\text { isoglosses }\end{array}$ \\
\hline salt & \#2644 *g-ryum & ru: & lə & lu & $\mathrm{xu} ; \mathrm{ru} \sim \mathrm{\gamma u}(\mathrm{SA})$ & $*$ Iu & BG səruə \\
\hline sand & - & səgai? & 6əgəroi & bai; balji (SM) & bali & *sə-gə-raj & IA bālu 'sand' \\
\hline seed & $\# 1019$ *sey & mət $\tilde{\varepsilon}:$ & tailoi & $\begin{array}{l}\mathrm{t}^{\mathrm{h}} \mathrm{e}-\mathrm{li} \sim \text { ăli } \sim \mathrm{t}^{\mathrm{h}} \mathrm{e}- \\
\text { dzo ma-la }\end{array}$ & Păsə & *mə-Өai & PTk * $t^{\mathrm{h}} \mathrm{a}$ \\
\hline sell & $\begin{array}{l}3376 *(\mathrm{~g} / \mathrm{m} / \mathrm{s}) \text {-lay } \\
\text { EXCHANGE/BARTER }\end{array}$ & la: & laəna $\sim \mathrm{k}^{\mathrm{h}} \mathrm{a}^{\mathrm{u}}$ & $\begin{array}{l}\text { la } a^{o} \sim p^{h} e^{h} o^{\sim} \\
\text { tJoyret }\end{array}$ & dıu & $* \mathrm{laC}$ & PK lou \\
\hline seven & Hruso: \#2505 s-ni-s & məwài? & mija & mija(k) & mıo; mrjo (SA) & ${ }^{*}$ mə-ljak & BG məlija: \\
\hline sew & \#2350 *krwi(y) & kàì & $\begin{array}{l}\text { galikhro } \\
k^{h} \text { romna }\end{array}$ & (grə-)khri $k^{h} r \partial$ & kfijo & ${ }^{*} \mathrm{k}(\mathrm{r}) \mathrm{iC}$ & - \\
\hline short & $\# 7175$ *s-n(i/u)n & $\begin{array}{l}\text { mərõn; ma }{ }^{31} \\
\text { ทon }^{55}(\mathrm{LD})\end{array}$ & mərun & marun arun & $\begin{array}{l}\text { PĕtJesa; } \\
\text { udu(sa) (SA) }\end{array}$ & *mə-nun & - \\
\hline shoulder & $\begin{array}{l}\text { \#1327 * pway ARM } \\
\text { (upper) / SHOULDER }\end{array}$ & (mə)pot6(i) & - & pastun (SM) & - & - & - \\
\hline sister (elder) & $\begin{array}{l}\text { \#1619 ma FEMALE / } \\
\text { MOTHER }\end{array}$ & ama: momoa & Pama & $\begin{array}{l}\text { amo amu; } \\
\text { momo (SM) }\end{array}$ & Pama & *mə-ma & - \\
\hline sit, stay & $\begin{array}{l}\# 1906 * m-(t / d) u(\mathrm{~g} / \mathrm{k}) \\
\text { SIT }\end{array}$ & $\overline{\mathrm{d} z \grave{u}}{ }^{\mathrm{T}}$ & gijon gijona & $\begin{array}{l}\text { deyradzun } \\
\text { dzu? } \sim \text { gijon }\end{array}$ & $\begin{array}{l}\text { xowe; y ro } \\
\text { (SA) }\end{array}$ & $* \overline{\mathrm{d} z} \mathrm{u} u \mathrm{C}$ & $\begin{array}{l}\text { BG duk; SS dyn; KD } \\
\text { dun }\end{array}$ \\
\hline six & $\# 2621$ *d-k-ruk & rè? & $\mathrm{ra}(\mathrm{m})$ & re(?) & xe; rije (SA) & *reC & BG rap \\
\hline skin & $\# 792 * p(r / y) a$ & mәрjа: & məpə məpre & məpre & Păt $\int^{h} \partial$ & *mə-prja & OC*pra m-paj \\
\hline sky & - & nədəlu: & gijan & (na)đ̄ang & $\begin{array}{l}\text { me } \overline{d z e} \text {; ne } \overline{d z \partial} \\
\text { (SA) }\end{array}$ & *nə-də-lan & $\begin{array}{l}\text { Bengni ñi-do:-mo: } \\
\text { (PT) }\end{array}$ \\
\hline sleep & $\# 128 * g-(d) z(i / u) m$ & $\overline{d z e}:$ & dzi(na) & dzi & $\begin{array}{l}\text { dıumudzo; } \overline{d 3} u \\
\text { (SA) }\end{array}$ & $* \overline{\mathrm{d} z} \mathrm{~V}$ & OC *ts ${ }^{\text {himp; }}$ SS džin \\
\hline smell (v) & $\begin{array}{l}\# 1416 \text { *s-rim; \#1415 } \\
\text { *m/s-nam }\end{array}$ & $r \tilde{\varepsilon}:$ & - & nen (SM) & sohu (SA) & - & - \\
\hline smoke (n.) & \#2361 *kəw-n/t & mekə: & maikh ${ }^{\mathrm{h}}$-n & maik $k^{h} ә n$ & mŭkJə & *maj-kən & - \\
\hline snake & \#2178 *bəw & bə: & bə ${ }^{u}$ & $\begin{array}{l}b^{\mathrm{h}} \mathrm{u} \sim \text { nabu } \sim \\
\text { nabəu }\end{array}$ & by & *bəw & - \\
\hline
\end{tabular}




\begin{tabular}{|c|c|c|c|c|c|c|c|}
\hline Gloss & PTB & Bangru & E. Miji & W. Miji & Hruso & $\mathrm{PH}$ & $\begin{array}{l}\text { Proto-forms, } \\
\text { isoglosses }\end{array}$ \\
\hline snow & - & tər $\tilde{\varepsilon}^{\prime}$ & - & dəlen (SM) & $\mathrm{t}^{\mathrm{h}}$ Ĩno (SA) & *dəren & $\begin{array}{l}\text { PCN *ro(?) } \\
\text { ICE/SNOW/HAIL }\end{array}$ \\
\hline soft, smooth & $\begin{array}{l}\text { \#2577 *pryaw-k SOFT } \\
\text { / BOILED }\end{array}$ & ləpəri: ləpri: & - & məbəlją (SM) & $\overline{\mathrm{d} z} \mathrm{u} ;$ nə (SA) & ${ }^{*}$ mə-lə-prjuC & - \\
\hline soil & - & nò? & na? & na? & no & *na? & $\begin{array}{l}\text { PT *mron EARTH; } \\
\text { Bengni ño:; BG nak; } \\
\text { SS k }{ }^{h} \text { ñ } \sim k^{h} \text { nu: }\end{array}$ \\
\hline speak & PTB *taj/w (prop.) & te: & ta $\sim$ reena & $\begin{array}{l}\text { Jo? } \\
\text { too? } \sim t^{\text {h }} \text { ro?; } \\
t^{\text {he }} e(n)(S M)\end{array}$ & $t \int^{h}$ edzo & $*$ təwC & $\begin{array}{l}\text { OC taw-s, Middle } \\
\text { Chinese tsyew }{ }^{H} ; \\
\text { Pekon Kayan tài } \\
\text { (MK); Luxi Leqi ta:i }{ }^{53} \\
\text { (TBL) }\end{array}$ \\
\hline spear & $\begin{array}{l}\# 2205 * m \text {-dun } \ m- \\
\text { dan }\end{array}$ & gəţəว & dzon d3o? & $\begin{array}{l}\text { dzun; } \overline{d z u n} \\
\text { (SM) }\end{array}$ & t $\int u d 3 u$ & *dzun & BG dzion; SS dzo \\
\hline spicy & - & sə: & məJu & 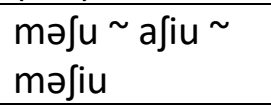 & nəsə; nəfə (SA) & *məsu & PTk *sa \\
\hline spittle & - & jè? & - & 3e (SM) & zem $\overline{\mathrm{d} z} \mathrm{i}$ (SA) & $*$ je? & - \\
\hline split & $\begin{array}{l}\text { \#5616 * pryak } \\
\text { SEPARATE / SPLIT }\end{array}$ & prje: pre: & - & - & - & - & - \\
\hline stab, punch & - & ko: & - & ran ran (SM) & $\widehat{k J i}(S A)$ & - & PK ko: 'hit' \\
\hline stand & - & gi: & gijon gəron & $\begin{array}{l}\text { gijon } \sim \\
\text { magarangi }^{u}\end{array}$ & gudzu & *gu & - \\
\hline star & - & 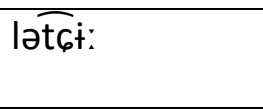 & $\begin{array}{l}\text { mətJonbijan } \\
\text { nitJobijon }\end{array}$ & dətJun & lătsə & *เə-ţun & $\begin{array}{l}\text { OC*s-ts }{ }^{h \varsigma} e n ; \text { PNN } \\
*_{\text {li:t STAR / MOON }}\end{array}$ \\
\hline steal & \#2365 *r-kəw & ləkə: & $\int \mathrm{jk}^{\mathrm{h}} \mathrm{au} \sim \mathrm{t} \int \partial \mathrm{k}^{\mathrm{h}} \partial$ & $\widehat{(\mathrm{t} f \mathrm{i}) \mathrm{k}^{\mathrm{h}} \partial}$ & 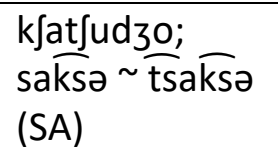 & *kə & $\begin{array}{l}\text { WT rku; BG } \\
\text { mekaukau; PLB } \\
\text { *kəw }^{2}\end{array}$ \\
\hline $\begin{array}{l}\text { storehouse, } \\
\text { granary }\end{array}$ & - & $\overline{\text { tढu: }}$ & - & ţun (SM) & - & *tढ़un & OC * ts ${ }^{\text {hs }}$ an \\
\hline
\end{tabular}




\begin{tabular}{|c|c|c|c|c|c|c|c|}
\hline Gloss & PTB & Bangru & E. Miji & W. Miji & Hruso & $\mathrm{PH}$ & $\begin{array}{l}\text { Proto-forms, } \\
\text { isoglosses }\end{array}$ \\
\hline straight & - & məkəro: & - & mugəraך (SM) & gədou (SA) & *mə-gə-ran & - \\
\hline suck & $\begin{array}{l}\text { \#259 *s-nəw(k/n) } \\
\text { BREAST/ MILK }\end{array}$ & pənun & - & bənu (SM) & - & *bə-nun & PK pənu: \\
\hline sun & - & $\overline{\mathrm{d} z \grave{u}} ?$ & d3o? & dzo?; zu? (SM) & dıu; $\overline{d z} u(S A)$ & $* \overline{d z} u ?$ & $\begin{array}{l}\text { Bengni do:-ñi, Bokar } \\
\text { dun-n̋i (PT) }\end{array}$ \\
\hline swallow & \#627 *mlyəw-k & pəlja pe: & - & bəlui (SM) & - & *bə-ljaC & PCN *m-lju(k) \\
\hline sweet & - & dzan & $\begin{array}{l}\text { mədzan } \\
\text { məzan }\end{array}$ & $\begin{array}{l}\text { adzan } \\
\operatorname{məd} z a(n / \eta) \sim \\
\text { mədzijan }\end{array}$ & $\frac{\mathrm{d} \int \partial ̆ \mathrm{mt} \int \mathrm{u} ;}{\mathrm{d} z \operatorname{im} t \int \mathrm{iu}(\mathrm{SA})}$ & *mə-jan & $\begin{array}{l}\text { PCN *m-yan SWEET; } \\
\text { Bugun } p^{h} \text { ijan }\end{array}$ \\
\hline swell & $\begin{array}{l}\# 111 *(p / b) \operatorname{lin} \text { FULL / } \\
\text { FILL }\end{array}$ & ləท & - & olin (SM) & - & $* \operatorname{lin}$ & - \\
\hline $\begin{array}{l}\text { (swidden) } \\
\text { field }\end{array}$ & - & wa: & - & vaw (SM) & - & *wa & - \\
\hline swim & - & dza: & $\begin{array}{l}\text { brəona } \\
\text { vəsanna }\end{array}$ & 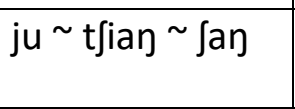 & $\begin{array}{l}\text { hudıo; } \overline{d z o} \\
\text { (SA) }\end{array}$ & $* \overline{\mathrm{d}} \mathrm{a} \mathrm{aC}$ & PT *bjan \\
\hline tail & $\begin{array}{l}\# 1288 * \text { r-may } \ m- \\
\text { ray }\end{array}$ & mələwja: & $\begin{array}{l}\text { mənəmə(re/rai } \\
\text { ) }\end{array}$ & $\begin{array}{l}\text { adəmai } ~ \\
\text { ademek } \sim \\
\text { mədərə; dimrai } \\
\text { (SM) }\end{array}$ & $\begin{array}{l}\text { Pexem; ərim } \\
\text { (SA) }\end{array}$ & *mə-lə-mrjaj & $\begin{array}{l}\text { OC mjweri; Mandarin } \\
\text { wěi }\end{array}$ \\
\hline take & \begin{tabular}{l}
$\# 376(\mathrm{~g} / \mathrm{m} / \mathrm{s})$-lay \} $\\
{(\mathrm{r} / \mathrm{s}) \text {-ley \く b-rey }} \\
{\text { EXCHANGE/ BARTER }}$ & lə: & ləna miniro & $\begin{array}{l}\text { lə †əجne } \\
\text { hotə; lug (SM) }\end{array}$ & lad3o & *ləw & $\begin{array}{l}\text { PT *lan; PKC *laa-I, } \\
\text { laak-II; TS la; KD la; } \\
\text { PK lei rei }\end{array}$ \\
\hline tell & - & tढatə & t $\int 0 \sim$ t foana & $\begin{array}{l}\mathrm{t}^{\mathrm{h}} \text { ro?mo } \sim \mathrm{t} \int \mathrm{ua} \\
\sim \mathrm{t}^{\mathrm{h}} \mathrm{e}\end{array}$ & tru; ${\widehat{t} \mathrm{f}^{\mathrm{h}} \mathrm{e}(\mathrm{SA})}$ & $*{ }_{\mathrm{t} 6 \mathrm{aC}}$ & - \\
\hline ten & PTB *run (prop.) & rəy & lən & tən lən & $x ə ;$ yə rə (SA) & *ran & PT *rjun; PTk *ra \\
\hline that & - & petci! & $\begin{array}{l}\text { pa't } \int i a ~ \\
\text { pa't } \int \partial l o\end{array}$ & 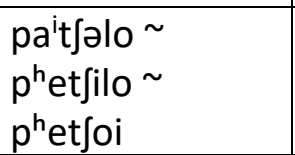 & $\mathrm{t}^{\mathrm{h}}$ ose & *paj-tढ़i & \\
\hline that (lower) & - & putढ $(.)$. & - & $p^{\text {hutsu }}$ (SM) & - & ${ }^{*}$ pu-ţढi & - \\
\hline
\end{tabular}
\end{tabular}




\begin{tabular}{|c|c|c|c|c|c|c|c|}
\hline Gloss & PTB & Bangru & E. Miji & W. Miji & Hruso & $\mathrm{PH}$ & $\begin{array}{l}\text { Proto-forms, } \\
\text { isoglosses }\end{array}$ \\
\hline thigh & - & maru: & - & $\operatorname{lu}(S M)$ & - & ${ }^{*}$ mə-lu & TS lum \\
\hline think & $\begin{array}{l}\text { \#6621 *məy WISH, } \\
\text { WANT (prov.) }\end{array}$ & mi: & - & mjen, Ju (SM) & - & - & $\begin{array}{l}\text { PK mi 'think, wish'; TS } \\
\text { mi 'think' }\end{array}$ \\
\hline this & - & həク & hunlo hãna' & huplo hupji & heji; hunlo (SA) & *hun & $\begin{array}{l}\text { PK həఐ; Galo ‘hi } \\
\text { (GLDC) }\end{array}$ \\
\hline thread & - & nù? nu: & kəbo & $\begin{array}{l}\text { kŭpo dzan } \\
\text { kəbo }\end{array}$ & məsin & - & - \\
\hline three & \#2666 *g-sum & kətẽ̃ & $\mathrm{k}^{\mathrm{h}}$ ətəm & $\begin{array}{l}\text { gətən } \sim \text { kət }{ }^{\mathrm{h}} \text { ən } \\
\sim \mathrm{k}^{\mathrm{h}} \text { ətəm }\end{array}$ & dzə; $\overline{d z a}(S A)$ & *gə-Өəm & - \\
\hline thorn & $\begin{array}{l}\# 2218 * m-(d) z(y) u(:) k \\
\text { PIERCE/ THORN }\end{array}$ & kəवृद̧̀े? & $\left(k^{h}\right.$ ə/gə)zau & 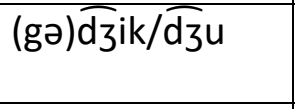 & kut $\widehat{u} u$ & *gə-वิdzuC & - \\
\hline thousand & - & 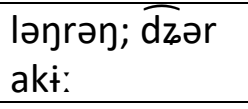 & hazari & hazari & hădzaxa & - & IA hazār \\
\hline tongue & $\# 621 * \mathrm{~m} / \mathrm{s}$-lay $\Varangle \mathrm{s}$-ley & məләり pəlja: & jak $^{h} e^{\sim}$ jak $^{h}$ ijoi & $\begin{array}{l}\text { dzakihi ja?k }{ }^{h_{i}} \\
\sim \text { lakfi; zeghi } \\
\text { (SM) }\end{array}$ & $\begin{array}{l}\text { Pedzabla; zebla } \\
\text { (SA) }\end{array}$ & *bə-laj & - \\
\hline tooth & \#3316 *tway (prov.) & matu: & tə & $t^{h} u \sim t u$ & Pet $^{h} u ;$ ət $\int u(S A)$ & $*_{\text {mə-taC }}$ & $\begin{array}{l}\text { PK kətuan; SS } \\
\text { nət }{ }^{h} y^{\sim} \text { nut } \\
\text { hintun; }\end{array}$ \\
\hline tree & - & gəne: & (ge/go)noi & $\begin{array}{l}\text { uni uni? } \\
\text { OPni; ou (SM) }\end{array}$ & Joni; jõn (SA) & *(g)o-naj & $\begin{array}{l}\text { lit. 'wood+mother' } \\
\text { viz. PK hıen-mua; KD } \\
\text { Gin-ama etc. }\end{array}$ \\
\hline turquoise & - & ju: & - & - & - & - & WT g.yu \\
\hline two & $\# 2504 *(\mathrm{~g} / \mathrm{s})-\mathrm{ni}-\mathrm{s}$ & kərei & kəran kherə & geni $\sim$ gərin & 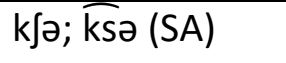 & *gə-niC & WT gnyis \\
\hline $\begin{array}{l}\text { uncooked } \\
\text { rice }\end{array}$ & - & $\tilde{\varepsilon}: \log \tilde{\varepsilon} \tilde{I}$ & namnəgam & andəgən an & Polgə & *an-lə-giN & $\begin{array}{l}\text { PT am-bunn; Rawang } \\
\mathrm{am}^{33} \text { (LP) }\end{array}$ \\
\hline vegetable & - & pe: & - & $\mathrm{p}^{\mathrm{h}} \mathrm{en}(\mathrm{SM})$ & $\mathrm{p}^{\mathrm{h}} \mathrm{ij} \varepsilon$ (SA) & *pen & - \\
\hline
\end{tabular}




\begin{tabular}{|c|c|c|c|c|c|c|c|}
\hline Gloss & PTB & Bangru & E. Miji & W. Miji & Hruso & $\mathrm{PH}$ & $\begin{array}{l}\text { Proto-forms, } \\
\text { isoglosses }\end{array}$ \\
\hline vomit & $\begin{array}{l}\text { \#1782 *mwa æ mya- } \\
\text { n SPIT/VOMIT }\end{array}$ & mu: & - & $\mathrm{mu}(\mathrm{SM})$ & - & ${ }^{*} \mathrm{mu}$ & - \\
\hline walk & PTB *daj (prop.) & dja: d & dai?na & $\begin{array}{l}\text { dai naida'du } ~ \\
\text { da'dewo } \sim \\
\text { dai?na }\end{array}$ & $\overline{\mathrm{d} z e}(\mathrm{SA}) ; \mathrm{d} 3 \mathrm{u}$ & $*$ daj & $\begin{array}{l}\text { TS dI; Dulong di }{ }^{53} \\
\text { (TBL) }\end{array}$ \\
\hline hot, warm & $\# 1844$ *tsa-t \ dza-t & 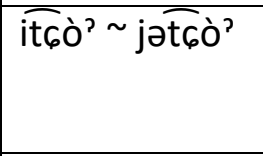 & $\begin{array}{l}\text { məd3u } \sim \text { məzi } \\
\sim \text { mələ }\end{array}$ & $\begin{array}{l}\text { adziu } \\
\operatorname{məd}(i) \text {; } \\
\text { məziu (SM) }\end{array}$ & 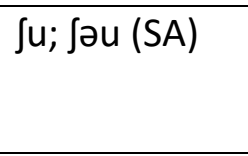 & *tढaC & $\begin{array}{l}\mathrm{PCN} \text { *tsha HOT; TS } \\
\text { ts }^{\text {halu }}\end{array}$ \\
\hline water & - & wi: & və & və & $k^{h} u$ & $*$ wi & $\begin{array}{l}\text { Guiqiong wi }{ }^{53} \text { (rain, } \\
\text { ZM); Jiulong Pumi } \\
\text { wi }^{35} \text { (TBL); PK kua; } \\
\text { BG, SS k }{ }^{\mathrm{h}} \text {; KD k } \text { k }^{\mathrm{h}}\end{array}$ \\
\hline waterfall & - & kuase( $(\eta)$ & - & - & $\overline{\text { ksətsu (SA) }}$ & ${ }^{*}$ kua-su? & PK kuasua? \\
\hline weave & $\# 2686 *(t / d) a k$ & $\widehat{\text { tsai } ~} \widetilde{\text { t6ai }}$ & $\begin{array}{l}\text { tfomna } \\
\text { gəitfom }\end{array}$ & $\begin{array}{l}\text { 6ən } \sim \mathrm{t}^{\mathrm{h}} \text { rən } ~ \\
\text { (grə/zan)trin }\end{array}$ & $\mathrm{t}^{\mathrm{h}_{\mathrm{ijo}}}$ & $*_{\text {traj }}$ & $\begin{array}{l}\text { OC *tək; WT thags; } \\
\text { KD dak }\end{array}$ \\
\hline what & - & təy & tən & $\begin{array}{l}\tan \sim \operatorname{tin} \sim \\
t^{\mathrm{h}} \text { en; } \mathrm{t}^{\mathrm{h}} \text { in }(\mathrm{SM})\end{array}$ & ha & $*$ tin & $\begin{array}{l}\text { SS ti; KD Pa ha; TS } \\
\text { han }\end{array}$ \\
\hline where & - & kə: & $k^{h} \partial k \sim k^{h}$ roi & $k^{h} ә j o \sim k^{h} i j a$ & $\begin{array}{l}\text { hagə; hago } \\
\text { (SA) }\end{array}$ & ${ }^{*} \mathrm{k} \partial ?$ & $\begin{array}{l}\text { PKC *koy } \ \text { khoy } \\
\text { hoy (prov.); KD } \\
\text { ha?kJ?; TM gat6 }{ }^{\text {h}} \partial\end{array}$ \\
\hline white & $\begin{array}{l}\text { \#1235 * plu WHITE/ } \\
\text { SILVER/MONEY }\end{array}$ & ləpu: & $\begin{array}{l}\text { mugram } \\
\text { məgijan }\end{array}$ & $\begin{array}{l}\text { mugram } \sim \\
\text { magəran } \\
\text { magəran }\end{array}$ & gro & *mə-gə-raN & - \\
\hline who & \#5632 *su (prov.) & ti:wo: & təə & $t \int^{h} u \sim t \int i u$ & dzu; $\overline{d z u}(S A)$ & $* \theta u$ & $\begin{array}{l}\text { PKC *tuu } \\
\text { RELATIVIZER (who, } \\
\text { which) }\end{array}$ \\
\hline wind & \#2412 *g-lәу & loa & jo & jo & Iau (SA) & *low & \\
\hline window & - & $\begin{array}{l}\text { i:rin; pa:run } \\
\text { pua:run }\end{array}$ & $k^{\text {hirki }}$ & $k^{\text {hirki }}$ & $k^{\text {hirki }}$ & - & Hin. khiḍki \\
\hline
\end{tabular}




\begin{tabular}{|c|c|c|c|c|c|c|c|}
\hline Gloss & PTB & Bangru & E. Miji & W. Miji & Hruso & $\mathrm{PH}$ & $\begin{array}{l}\text { Proto-forms, } \\
\text { isoglosses }\end{array}$ \\
\hline wing & $\# 731{ }^{*} \mathrm{~g}-(\mathrm{t})$ syəw-k/n & mətढ़े? & - & gət $\widehat{\text { fi }}(\mathrm{SM})$ & - & *gə/mə-tढे & - \\
\hline with & - & dदৈərə & - & dzoru (SM) & -dza (SA) & $* \frac{\mathrm{d} z \mathrm{~V}}{\mathrm{~d} u}$ & - \\
\hline wood & - & go: & go ge & $\mathrm{u} \sim \mathrm{o}$ ? & So & $*(\mathrm{~g}) \mathrm{o}$ & - \\
\hline woman & - & nəwย: & nəmra ${ }^{i}$ & $\begin{array}{l}\text { nəmeg } \\
\text { nəmrai } ~ \\
\text { numazə }\end{array}$ & $\operatorname{mim}(i)$ & ${ }^{*}$ nəməraj & $\begin{array}{l}\text { PT *mji-mə: } \\
\text { WOMAN; Koro mimi } \\
\text { (AK) }\end{array}$ \\
\hline year & $\begin{array}{l}\# 2501 * s-n i(:) \eta \succ s- \\
\text { nik }\end{array}$ & anəクnəク & dəran & dure(n) & $\begin{array}{l}\text { Podze; lidz̃o } \\
\text { (SA) }\end{array}$ & $*$ də-nin & $\begin{array}{l}\text { OC *C.n`in 'harvest; } \\
\text { year'; WT na ning } \\
\text { 'last year'; TS nin }\end{array}$ \\
\hline yesterday & - & təga: & təganogo & degau & $\phi u ;$ fwu (SA) & *də-gaC & $\begin{array}{l}\text { Hruso 'yesterday' = } \\
\text { Miji wu 'day', Bangru } \\
\text { agu 'day' }\end{array}$ \\
\hline $\begin{array}{l}\text { younger } \\
\text { sibling }\end{array}$ & \#2492 *na:w & mərə: & ran ra: & (mə/na)nu & Ponu; niu (SA) & *mə-nun & $\begin{array}{l}\text { PCN *nu; SS mirin } \\
\text { 'younger sister of } \\
\text { man'; PCN *nu; Koro } \\
\text { ne (AK) }\end{array}$ \\
\hline $1 \mathrm{SG}$ & $\begin{array}{l}\text { \#2530 PTB *na-y } \Varangle \\
\text { *ka l/ME/SELF }\end{array}$ & no: & nijan & $\begin{array}{l}\text { nijan nijan; } \\
\text { nan (SM) }\end{array}$ & no & $*^{*}$ na(-jan) & PT *no: 'I' \\
\hline $2 \mathrm{SG}$ & \#2489 *nan THOU & ni: ni: & ni & ni & $b^{h} a$ & $*_{n i}$ & OC *na? \\
\hline $3 \mathrm{SG}$ & - & petढ़i & $a^{i}$ & $\mathrm{i} \sim \mathrm{p}^{\mathrm{h}} \widetilde{\mathrm{et} \int \mathrm{u}}$ & Pi & $*$ ?i & TM Pi \\
\hline $1 \mathrm{DU}$ & - & ka:re: & - & - & - & - & - \\
\hline $1 \mathrm{PL}$ & *nəy & kani: kani: & ani animihõy & ani anijaahun & ni; ani (SA) & *ka-ni & PNN *nəy \\
\hline $2 \mathrm{PL}$ & - & ni:; $\overline{\mathrm{d} z e} \mathrm{e}$ & $\begin{array}{l}\text { dzimihõn } \\
\text { dzei }\end{array}$ & $\begin{array}{l}\text { dzi ina } \\
\text { dziməJun }\end{array}$ & d3o & $* \bar{d} \bar{c} V$ & WT khyed \\
\hline $3 P L$ & - & $\begin{array}{l}\text { dze:(mələџ/ } \\
\text { kadi) }\end{array}$ & $a^{i} r a$ & $\begin{array}{l}\text { inamehijan } \\
\mathrm{p}^{\text {haina }} \sim \\
\text { airaahun }\end{array}$ & na & $*$ na & $\begin{array}{l}\text { WT kho-na, mo-na } \\
\text { 3SG }\end{array}$ \\
\hline
\end{tabular}

